\title{
36TH ANNUAL SCIENTIFIC MEETING OF THE CANADIAN PAIN SOCIETY: ABSTRACTS
}

\section{THURSDAY, MAY 21, 2015 SCIENTIFIC PROGRAM, DAY 1}

\section{9:00 - 9:45am}

\section{1}

KEYNOTE SPEAKER: MARY ELLEN JEANS LECTURE UNDERSTANDING HUMAN PAIN PERCEPTION AND ANALGESIA THROUGH ADVANCED NEUROIMAGING Invited Speaker: Irene Tracey Nuffield Professor Anaesthetic Science \& Director, Oxford Centre for FMRI of Brain, Nuffield Department of Clinical Neurosciences, (Head, Nuffield Division Anaesthetics), Oxford University, England, UK The ability to experience pain is old and shared across species. It confers an evolutionary advantage and provides a warning of harm or impending threat. As far back as Hippocrates, it was understood that the brain was key to a person experiencing pain. Fortunately, these days we now have many techniques available to explore the human central nervous system in vivo from a functional, structural and chemical perspective in both patients and healthy subjects. Relating specific neurophysiologic measures to perceptual or non-perceptual changes induced by peripheral or central sensitisation, behavioural, psychological or pharmacological mechanisms and identifying their site of action within the CNS has both value and has been a major goal for scientists, clinicians and the pharmaceutical industry. Identifying non-invasively where functional and structural plasticity, sensitisation and other amplification or attenuation processes occur along the pain neuraxis for an individual and relating these neural mechanisms to specific pain experiences, measures of pain relief, persistence of pain states, degree of injury and the subject's underlying genetics, has neuroscientific relevance and potential diagnostic value.

Learning Objectives:

1. Better knowledge of the range of physiological measures available using advanced neuroimaging that give novel insights into central pain mechanisms

2. To understand the importance of the descending pain modulatory system in acute and chronic pain

3. To learn how current theories regarding how the brain generates perception can inform the pain field.

\section{Session 101: 11:00am - 12:30pm}

\section{2}

THERE IS AN APP FOR THAT: USING MOBILE TECHNOLOGY TO IMPROVE CHRONIC PAIN

Chair: Jennifer Stinson

The Hospital for Sick Children, Toronto, Ontario

Speakers: Robert N Jamison, M Cary Reid Jr, Jennifer Stinson

Workshop Objective:

This symposium will detail the content, face validity, reliability, usability, and technical issues associated with the use of pain apps. Challenges with developing these apps and future areas for research will also be discussed.

Learning Objectives:

1. Describe the clinical challenges associated with using smartphone pain apps for remotely monitoring and managing chronic pain.

2. Outline empirical approaches for using smartphone pain apps in different clinical settings with different patient populations.

3. Discuss future areas of research designed to improve compliance and remote data collection among chronic pain patients.

\section{A}

\section{MOBILE TECHNOLOGY FOR MANAGEMENT OF CHRONIC PAIN: DEVELOPMENT AND IMPLEMENTATION OF A SMARTPHONE PAIN APP}

Robert N Jamison

Brigham and Women's Hospital, Boston, Massachusetts, USA

There has been an explosion of mobile devices that have been used to track health data and change the approach to management of chronic diseases. It is estimated that there are nearly 15,000 health and fitness apps now available, many of which are designed to help persons with chronic pain. In the US and Canada, 85 percent of adults have access to a cell phone, of which half are smartphones capable of going online and running sophisticated apps. These devices allow data to be transferred to healthcare professionals and can offer interventions to a greater number of patients than could be seen individually. A critical barrier to incorporation of technology in delivering behavioral treatments for patients learning to live with chronic pain are outcome studies that demonstrate improvement of care with a decrease in healthcare utilization. Dr Jamison will review the literature on the use of smartphone pain apps to help manage chronic pain patients' conditions remotely and that have the potential to decrease healthcare utilization. He will detail the content, face validity, reliability, usability, expense, and technical issues associated with the use of pain apps and describe findings from a study of patients with cancer and noncancer chronic pain who are using a smartphone pain app.

\section{B}

\section{KEY ISSUES RELATED TO MHEALTH AND PAIN CARE IN LATER LIFE \\ M Cary Reid Jr \\ Weill Cornell Medical College, New York, New York, USA}

In recent years, mobile devices have become increasingly lightweight, affordable, and user-friendly, and they are now widely used by older people. In combination with targeted prompts to record subjective states (e.g., pain levels), these devices offer near-continuous sampling capability for variables such as daily activity level, stress, mood, social networks, and sleep habits. Moreover, without any input from the individual, mobile phones can automatically collect information about time, location, voice quality as a measure of stress, and even the people around individuals. Many mobile phones have built-in accelerometers that can generate data on movement (pace, gait, distance) and sleep patterns. These devices could potentially improve patient care through more effective patient monitoring of treatment outcomes, enhanced patient-provider communication, and by providing new ways to deliver treatment. Dr Reid will review barriers and facilitators to the use of pain apps in the care of older adults who may benefit greatly from the use of these devices given age-related disparities in pain care. He will also present results from a recently completed study which demonstrated that an SMS-text message based social support intervention delivered via mobile phone can reduce pain and pain interference levels in patients with chronic pain.

\section{C}

PAINSQUAD: A SMARTPHONE APP FOR "JUST-IN-TIME" MONITORING AND MANAGEMENT OF PEDIATRIC CANCER PAIN

Jennifer Stinson

The Hospital for Sick Children, Toronto, Ontario

Despite advancements in the amount and quality of pain research, adolescents with pain due to cancer and/or its treatment continue to report it as poorly manage. Achieving the best possible pain management requires collaboration between adolescents with cancer and healthcare providers to: (a) measure pain severity and impact, (b) empower adolescents to take on pain self-care through imparting knowledge and advice, (c) deliver and evaluate pain-focused interventions, and (d) provide ongoing support. Currently, this process occurs mainly during brief clinic 
visits or busy hospital admissions. However, due to important advancements in therapeutic regimes and changes to healthcare systems, these youth now spend less time hospitalized and more time at home. This means youth with cancer and their families are increasingly responsible for managing cancer-associated pain at home with minimal supervision by healthcare provider. With emerging interactive and communication technologies, new media such as smartphones are available for the delivery of health interventions. Dr. Stinson will discuss the development, usability and feasibility testing, and psychometric testing of a new iPhone app for youth with cancer called the Pain Squad ${ }^{\mathrm{TM}}$ that incorporates gamification. She will also discuss the development of PainSquad+, which employs a clinical care algorithm to provide adolescents with cancer with real-time pain management support.

\section{Session 102: 11:00am - 12:30pm}

\section{3}

\section{UNDERSTANDING SELF-REGULATORY PROCESSES IN} ABDOMINOPELVIC PAIN: WE HAVE THE ANSWERS?

\section{Chair: Dean Tripp}

Queen's University, Kingston, Ontario

Speakers: Dean Tripp, Adrijana Krsmanovic, Abi Muere

Workshop Objective:

The overall aim is to expand upon the current literature on abdominopelvic pain, with a particular focus on Inflammatory Bowel Disease (IBD), Chronic Prostatitis/Chronic Pelvic Pain Syndrome (CP/CPPS), and Interstitial Cystitis/Bladder Pain Syndrome (IC/BPS). The cutting edge research has lagged behind on in-depth discussions of self-regulatory processes and the impact of psychosocial factors on pain and patient outcome. This symposium will discuss how self-regulatory processes function in these abdominopelvic pain conditions. The discussions and the models that may be applied to the treatment of poor patient outcomes will engage researchers and clinicians alike.

Learning Objectives:

1. To develop awareness of the abdominopelvic pain literature on pain, appraisals, and coping.

2. To consider the relationships between pain and psychosocial variables in abdominopelvic pain conditions.

3. To be able to discuss variables of interest in patient outcomes and clinical targets for interventions and pain management.

\section{A}

\section{MECHANISMS IN THE RELATIONSHIP BETWEEN CATASTROPHIZING AND PAIN IN INFLAMMATORY BOWEL DISEASE (IBD)}

\section{Dean Tripp}

Queen's University, Kingston, Ontario

Inflammatory Bowel Disease (IBD), made up of Crohn's Disease and Ulcerative Colitis (UC), is a painful chronic gastrointestinal disease characterized by inflammation. Similar to other visceral chronic pain conditions, catastrophizing is associated with pain among IBD patients. Using a moderated mediation model, the present study examined the mechanisms through which catastrophizing predicts pain reports. 278 patients diagnosed with IBD were recruited from tertiary care urology clinics and completed questionnaires (demographics, McGill Pain Questionnaire, Pain Catastrophizing Scale, Brief Chronic Pain Coping Inventory, Patient Health Questionnaire). A moderated mediation model was investigated to test if illness-focused behavioural coping and wellness-focused behavioural coping each mediated the relationship between catastrophizing and pain, and if depressive symptomology moderated their effects. Catastrophizing had a direct effect on pain, $\beta=0.16, S E=0.045, p<0.001$. Illness-focused behavioural coping $(\beta=0.19, S E=0.040)$ and wellness-focused coping $(\beta=0.017, S E=0.010)$ both mediated the catastrophizing-pain relationship. The mediating effect of illness-focused coping was significantly stronger for participants with greater depressive symptomology; index of moderated mediation was 0.057 (95\% confidence interval: 0.025 to 0.097 ). These results indicate that the relationship between catastrophizing and pain is partially mediated by illness-focused and wellness-focused behavioural coping strategies. Furthermore, the mediating effect of illness-focused coping strategies is significantly stronger for individuals with greater depressive symptomology. These findings suggest the management of IBD pain may be supplemented through targeting specific psychosocial factors (appraisals \& coping).

\section{B}

\section{CATASTROPHIZING AND BEHAVIOURAL COPING AS PREDICTORS OF PHYSICAL AND MENTAL QUALITY OF LIFE IN CHRONIC PROSTATITIS/CHRONIC PELVIC PAIN SYNDROME (CP/CPPS)}

\section{Adrijana Krsmanovic}

Queen's University, Kingston, Ontario

Chronic Prostatitis/Chronic Pelvic Pain Syndrome (CP/CPPS) is a prevalent, refractory condition characterized by pain in the pelvic area and urinary frequency, largely unresponsive to medical interventions. The present study aimed to examine how catastrophizing and behavioural coping predict physical and mental Quality of Life (QoL) in this population. 175 patients from tertiary care clinics completed questionnaires. Demographics included age, ethnicity, education and employment status. Pain and symptoms were measured using the McGill Pain Questionnaire (SF-MPQ) and the Chronic Prostatitis Symptom Index (NIH-CPSI). Physical and mental QoL was measured using the Medical Outcomes Short Form 12-Item Health Survey (SF12); Chronic Pain Coping Inventory (CPCI) measured use of behavioural coping strategies and Pain Catastrophizing Scale (PCS) measured catastrophic thinking. Hierarchical regression modelling was conducted to examine the unique effects of appraisals and coping on patient QoL outcomes. Psychosocial variables accounted for a significant amount of variance over and above demographic and medical variables for both physical $\left(\Delta R^{2}=0.25\right.$, $p<0.01)$ and mental $\left(\Delta R^{2}=0.15, p<0.01\right)$ QoL. Guarding, resting, seeking social support and rumination were significant predictors of physical QoL, while resting and helpless catastrophizing were significant predictors of mental QoL. Catastrophic appraisals (rumination, helplessness) and behavioural coping strategies (guarding, resting and seeking social support) are predictive of poorer mental and physical QoL in men with CP/CPPS. The results indicate that these are variables of interest in patient outcomes, and they may be important targets for interventions aimed to improve patient $\mathrm{QoL}$.

\section{C} PSYCHOSOCIAL MEDIATORS AND MODERATORS OF THE
CATASTROPHIZING-AFFECTIVE PAIN RELATIONSHIP IN
INTERSTITIAL CYSTITIS/BLADDER PAIN SYNDROME (IC/BPS) INTERSTITIA
Abi Muere

Queen's University, Kingston, Ontario

Interstitial Cystitis/Bladder Pain Syndrome (IC/BPS) is a chronic pelvic pain syndrome characterized by cycling pain localized to the bladder and urologic symptoms of urgency, frequency, and dysuria (Nickel et al., 2009). There is an established relationship between catastrophizing and pain among IC/BPS patients (Tripp, Nickel, et al., 2006; Tripp et al., 2012). The present study aimed to identify the mechanisms through which catastrophizing predicts pain using a moderated mediation model. 341 women diagnosed with IC/BPS were recruited from tertiary care urology clinics and completed questionnaires (McGill Pain Questionnaire, Pain Catastrophizing Scale, Brief Chronic Pain Coping Inventory, Centre for Epidemiologic Studies Depression Scale). A moderated mediation model was examined to test if illness-focused behavioural coping strategies mediated the relationship between catastrophizing and affective pain, and if depression moderated this effect. Catastrophizing had a direct effect on affective pain, $\beta=0.40, S E=0.054, p<0.001$. Illness-focused behavioural coping was a significant mediator of the catastrophizing-affective pain relationship, $\beta=0.061, S E=0.019$. The mediating effect of illness-focused coping was significantly stronger for participants with greater depressive symptomology $(\beta=0.09, S E=0.031)$ in comparison to participants with less depressive symptoms $(\beta=0.04, S E=0.016)$; index of moderated mediation was 0.049 (95\% confidence interval: 0.0004 to 0.11 ). The relationship between catastrophizing and affective pain is partially mediated by 
illness-focused behavioural coping strategies. Importantly, this mediating effect is significantly stronger for individuals with greater depressive symptomology (i.e., moderated model). These findings have the potential to improve programs for the management of IC/BPS pain through targeting specific psychosocial factors associated with pain.

\section{Session 103: 11:00am - 12:30pm}

\section{4}

NOVEL SOLUTIONS TO A CHRONIC PROBLEM: IMPROVING PATIENTS' TRIAGING AT TERTIARY PAIN CLINICS

\section{Chair: Yoram Shir}

The Alan Edwards Pain Management Unit, McGill University Health Centre, Montreal, Quebec

Speakers: Alexander J Clark, Chris Spanswick, Yoram Shir

Workshop Objective:

Waiting time for treatment at chronic pain clinics in Canada is still suboptimal, especially in public tertiary treatment facilities. Consequently, clinics across Canada are creating novel triage systems, resulting in better triage methods and a significant shortening of waiting time. In this symposium we present different approaches successfully implanted in tertiary pain clinics in three different provinces in Canada.

Learning Objectives:

1. To review the triage challenges facing tertiary pain clinics in Canada

2. To describe the establishment of novel triage systems

3. To review preliminary outcomes of the new triage programs

\section{A}

\section{CENTRAL TRIAGE AND IMPROVING THE CONSULTATION} PROCESS FOR PATIENTS WITH CHRONIC PAIN

Alexander J Clark

Pain Management Unit, Capital Health and Dalhousie University, Halifax, Nova Scotia

Most publicly funded chronic pain programs in Canada have prolonged wait times from referral to assessment. In 2010 the program at Capital Health in Halifax consisted of three sites with wait times from referral to initial assessment varying from 24 months to more than 48 months. Each site maintained its own referral/triage process and was protective of this process since physicians wanted to see patients referred to them by their local colleagues. This session will describe the impact of the development and implementation of a central triage process in 2011 whereby all referrals are triaged by a nurse and administrative assistant (with the assistance of a pain physician when needed). The waitlist at each site was centralized to form a single waitlist and duplicate referrals to more than one site were identified. Patients who had been on a waitlist for more than 36 moths often were difficult to contact, had already been seen at a different site or did not want to be seen. This adjustment subsequently led to a 9 month overall reduction in the waitlist. Where possible, patients referred to a specific pain physician are seen by that physician but patients are given the option to see another physician in the program if a more rapid assessment is possible. Two innovative processes will be highlighted to provoke discussion; providing recommendations to referring physicians by fax or email and physician (referring) to physician (pain) telephone consultations. Both processes can be initiated immediately after receipt of a referral and can lead to enhanced patient care.

\section{B}

\section{GETTING THE PATIENT TO THE RIGHT PLACE:} AN EVOLVING SYSTEM

\section{Chris Spanswick}

University of Calgary, Calgary, Alberta

The Calgary Pain Program was set up in 2004. Its remit was to" provide integrated, coordinated quality pain services to the citizens of Region 3, Alberta". Other goals included developing a seamless continuum of pain services, ensuring equitable access. Addressing this required close collaboration with Primary Care, in particular Primary Care Networks (PCNs), in developing a system that responds to the needs of both the patient and the Family Physician. This session will describe the evolution of the referral system. The initial system included a single point of entry with strict criteria as well as the requirement for patients to complete an extended set of questionnaires. An $\mathrm{RN}$ screened all referrals and questionnaires to determine the next steps and the speed with which the patient would be seen. The options varied from an urgent contact with the Family Physician if patients expressed suicidal ideation, through offers of a telephone consult to a full team assessment. The system has evolved over the last few years as our collaboration with all of the PCNs in Calgary has progressed. Most of the PCNs now have some form of "Pain Program" which is affiliated with the Calgary Pain Program. A system of allowing patients to move seamlessly between services according to the patient's need is currently in the early stages of development. It is likely that the system may progress to one in which patients are initially referred to their local PCN service and moved centrally according to need and resources.

\section{C}

\section{IMPLANTATION OF A NEW TRIAGE SYSTEM OF PATIENTS REFERRED TO THE PAIN UNIT AT MCGILL UNIVERSITY HEALTH CENTRE}

Yoram Shir

The Alan Edwards Pain Management Unit, McGill University Health Centre, Montreal, Quebec

Similar to other tertiary pain centres, we traditionally faced the challenges of incomplete referrals, no formal triage guideline, long wait times and disorganization of the wait list. We first attempted to improve this situation by sending medical questionnaires to patients upon receipt of referral but this was not successful. Consequently, we developed a novel model of triage that was first sent for feedback to most major Canadian Pain Management Units. We then finalized our program based on the comments we received. Our process is now the following: Incoming referrals are reviewed daily by a nurse-clinician who identifies urgent referrals. These referrals bypass the rest of the process and are booked in priority. The remaining patients attend an orientation session within two weeks to complete medical and administrative questionnaires. This multimedia session also serves to introduce our clinic and its procedures, to initiate pain education and to explain the triage process. In addition we developed a triage prioritization hierarchy based on patients' medical, social, psychological and employment status. Triage is then done by multiple team members and the results are communicated to both the referral source and the patient. Surprisingly this new process has been well-received by patients, despite concerns that they would be upset at having to come from afar for a non-medical session. This new process has contributed to a significant reduction of our wait times. This workshop will explore the referral management process including the creation of a triage hierarchy incorporating bio-psycho-social factors.

\section{Session 104: 11:00am - 12:30pm}

\section{5}

OPTOGENETIC APPROACHES TOWARDS STUDYING PAIN

Chair: Gerald W Zamponi

University of Calgary, Calgary, Alberta

Speakers: Steve Prescott, Philippe Séguéla, Gerald W Zamponi

Workshop Objective:

In this session, speakers highlight the use of optogenetic techniques to examine the function of primary afferent neurons, and to probe the circuits that are involved in more complex processing of pain related information in the brain. Learning Objectives:

1. Appreciate the utility of optogenetics in studying the mechanistic underpinnings of pain.

2. Learn about recent advances in understanding pain processes gained through optogenetic approaches.

3. Gain knowledge about novel cellular and molecular targets for pain therapy. 
5A

\section{OPTOGENETIC INVESTIGATION OF SOMATOSENSORY ENCODING}

Steve Prescott

The Hospital for Sick Children, Toronto, Ontario

Sensation depends on how sensory information is encoded. Disrupting the encoding process can result in allodynia and hyperalgesia. But what aspect of the encoding process gets disrupted? Somatosensory information is first encoded by primary afferents through a two-step process: transduction of physical stimuli into receptor potentials and transformation of receptor potentials into spike trains. Isolating those two steps and studying them in situ has proven extremely difficult because the entire process occurs in the distal endings of primary afferent fibers - fibers that are too small to record from intracellularly. We have overcome those technical difficulties by developing a new optogenetic approach that involves expressing a lightactivated ion channel called channelrhodopsin-2 (ChR2) in afferent fibers of transgenic mice. Light is delivered to ChR2-expressing fibers in a precise spatiotemporal pattern to probe how action potentials, or spikes, are generated, thus enabling direct investigation of the transformation step. By comparing responses to photostimulation with responses to mechanical or other stimuli, we can also start to probe the transduction step. Using this novel ChR2-based photoactivation strategy, I will illustrate how colorectal afferents differ in their transformation properties and how those transformation properties are altered by inflammation. Specifically, I will show how "silent", mechanically insensitive nociceptors can be unsilenced through changes in their transformation properties. Contrary to the prevailing assumption that transduction dictates how somatosensory stimuli are encoded, these results highlight the importance of the transformation step and illustrate an innovative way to explore the entire encoding process and its pathological disruption.

\section{B}

\section{OPTICAL CONTROL OF PERIPHERAL PAIN PATHWAYS FOR EFFECTIVE ANALGESIA}

Philippe Séguéla

McGill University, Montreal Neurological Institute and Hospital, Montreal, Quebec

Peripheral nociceptors are specialized primary sensory neurons activated by noxious stimuli. Their selective activation and/or inhibition using optogenetics provides a direct way to control pain perception. Using a conditional genetic strategy, we produced murine models in which pain is either optically evoked by activation of excitatory channelrhodopsin-2 in nociceptors or inhibited by silencing nociceptor activity using hyperpolarizing opsins. Our silencing approach consists of expressing the proton pump archeorhodopsin (Arch) in Nav1.8+ nociceptors. Cellular distribution of the Arch-EGFP actuator, assessed in fluorescence, showed selective expression in Nav1.8+ sensory neurons. Strong labeling in laminae I and II of spinal cord and in fibers in glabrous skin demonstrated an efficient trafficking of the opsin from cell soma to both central terminals and peripheral innervation targets. Electrophysiological recordings confirmed significant outward photocurrents and hyperpolarizations in Arch-expressing cultured DRG neurons in response to stimulation with yellow light $(589 \mathrm{~nm})$. Light-evoked inhibition was sufficiently large to block electrically- as well as chemically-induced action potentials. In vivo analgesic effects of transdermal illumination with yellow light were observed in freely moving transgenic mice under acute, inflammatory and neuropathic pain conditions. Our results validate the development of constitutive transgenic mice in which the function of genetically-defined neuronal populations in somatosensory pathways can be effectively controlled non-invasively with high spatiotemporal resolution. Optical on/off switching of specific peripheral sensory components will facilitate the identification of candidate cellular targets for the treatment of intractable chronic pain.

\section{$5 \mathrm{C}$}

\section{OPTOGENETIC APPROACHES TO UNDERSTANDING CHRONIC PAIN}

Gerald W Zamponi

University of Calgary, Calgary, Alberta

During the development of chronic pain, plastic changes occur in both the peripheral and central nervous systems. In primary afferent neurons, nerve injury of peripheral inflammation causes dysregulation of a range of ion channels that then give rise to excessive afferent fiber excitability, and enhanced neurotransmission at synaptic terminals in the spinal dorsal horn. This includes an upregulation of Cav3.2 T-type calcium channels. We have recently shown that this enhancement of Cav3.2 channels is mediated by increased activity of the de-ubiquitinating enzyme USP5. We now show by transcutaneous optogenetic activation of TRPV1expressing nociceptors that increased neuronal activity is sufficient to induce persistent pain hypersensitivity via and activity-dependent upregulation of USP5 and thus increased T-type channel activity. Nerve injury also causes channels in carious cortical regions linked to sensory and emotional aspects of pain. We used optogenetic techniques to dissect the role of the medial prefrontal cortex in these processes.

Session 105: 1:30-3:00pm

\section{6}

WHEN SEX IS PAINFUL: TRANSLATING RESEARCH INTO CLINICAL PRACTICE FOR COUPLES WITH VULVODYNIA

Chair: Natalie O Rosen

Dalhousie University, Halifax, Nova Scotia

Speakers: Kate Rancourt, Natalie O Rosen, Serena Corsini-Munt

Workshop Objective:

Provide an overview of current research on the biopsychosocial factors involved in vulvodynia, and the translation of basic clinical research on interpersonal factors and pain acceptance into intervention research for couples affected by this condition.

Learning Objectives:

1. Describe the interpersonal context of vulvodynia and discuss recent research on the role of dyadic sexual communication in women and partners' pain and sexual outcomes.

2. Understand the role of pain acceptance on pain, psychological, and sexual outcomes in couples where the woman has vulvodynia, and describe the unique contribution of the partner's pain acceptance on the couple's outcomes.

3. Understand pain management and sex therapy strategies that show preliminary efficacy in the treatment of vulvodynia for women and their partners, and describe considerations for clinical testing of a newly developed psychological treatment option for couples with PVD.

\section{A}

\section{LET'S TALK ABOUT SEX: THE ROLE OF SEXUAL COMMUNICATION IN COUPLES' PAIN EXPERIENCE AND SEXUAL ADJUSTMENT TO VULVODYNIA}

\section{Kate Rancourt}

Dalhousie University, Halifax, Nova Scotia

Provoked vestibulodynia (PVD) is a prevalent subtype of vulvodynia (chronic vulvo-vaginal pain) that is most frequently triggered through sexual intercourse. PVD provides a unique setting in which to study the social context of pain because of the partner's role in provoking the pain during sexual activities. To better understand this interpersonal context, clinical researchers have applied concepts from the social psychological literature on romantic relationships to couples experiencing PVD. Sexual communication positively contributes to the sexual relationships of nonclinical couples. In two studies that examined sexual communication in PVD, women with PVD and their male partners reported significantly lower sexual communication than their pain-free counterparts. These findings highlight potential impairments in sexual communication for affected couples, though the impact of these deficits on women's pain and couples' sexual adjustment remains unclear. This presentation will discuss recent 
research that extended these results by examining sexual communication from a dyadic perspective. Results indicated that greater sexual communication in women and partners was associated with their own higher sexual functioning and sexual satisfaction, as well as their partner's higher sexual satisfaction. Women and partners reporting greater sexual communication also reported higher perceptions of women's self-efficacy for managing their pain. Additionally, when a woman's partner reported greater sexual communication, she reported lower pain during intercourse. Results point to the potential influence of sexual communication in modifying the pain experience of women with PVD, and impacting on couples' sexual wellbeing. Clinical implications for health care providers working with this population will be discussed.

\section{B}

LIVING A VALUED LIFE DESPITE PAINFUL INTERCOURSE: ACCEPTANCE OF CHRONIC PAIN IN WOMEN WITH VULVODYNIA AND THEIR PARTNERS

\section{Natalie O Rosen}

Dalhousie University, Halifax, Nova Scotia

Acceptance of chronic pain refers to openness to experiencing pain sensations and the pursuit of a satisfying life despite having chronic pain. While greater pain acceptance has been associated with positive functional outcomes in numerous chronic pain conditions, this construct has only been examined in the individual experiencing the chronic pain, despite widespread appreciation for the social dynamics of chronic pain. This presentation will describe the role of vulvo-vaginal pain acceptance on several outcomes from the perspective of both the woman and her partner in couples where the woman has provoked vestibulodynia (PVD). Results of this research indicate that women's greater pain acceptance was associated with lower self-reported pain during intercourse. Greater pain acceptance in women was associated with women's decreased anxiety and depression, and increased sexual satisfaction. Greater partner pain acceptance was related to increased partner sexual functioning. In addition, women in couples where both members endorsed low levels of pain acceptance reported significantly higher pain during intercourse than couples where one or both members reported high levels of pain acceptance. While cognitive-behavioural and medical treatments for PVD have been found to reduce women's pain and improve associated psychosexual impairments, some pain typically persists following treatment for many women. Thus, acceptance may be an important construct for improving women's psychological and sexual well-being in the presence of continued pain, as well as possibly decreasing the pain itself. Implications for the inclusion of acceptance-based treatment approaches for couples with PVD will be discussed.

\section{C}

\section{FROM RESEARCH TO CLINICAL PRACTICE: THE} DEVELOPMENT AND CLINICAL TESTING OF A COGNITIVEBEHAVIORAL COUPLE THERAPY FOR VULVODYNIA

\section{Serena Corsini-Munt}

Université de Montréal, Montréal, Québec

Vulvodynia has a $7-8 \%$ prevalence rate. Provoked vestibulodynia (PVD), the most common form of vulvodynia, is associated with psychosexual difficulties such as decreased sexual function, sexual frequency and pleasure for both women and their partners. Moreover, interpersonal factors such as partner distress, intimacy, sexual communication, and pain catastrophizing and pain acceptance of both women and their partners, are associated with women's pain and sexuality outcomes. Despite the interpersonal context in which PVD pain most often occurs, the woman, as the identified patient is often treated on her own with the pain being the sole treatment target. Given that psychological treatments have demonstrated comparable success to several medical treatment options for women with PVD, cognitivebehavioural therapy presents as a promising non-invasive treatment option that can target pain, pain-related sequelae, and sexuality outcomes for both women and their partners, while also including the partner in treatment. This presentation will provide an overview of the development and testing of a novel, empirically-based Cognitive Behavioural Couple Therapy (CBCT) for PVD. It will include a presentation of results and implications from its pilot-testing, and the development and launch of a bi-centre, randomized clinical trial comparing CBCT to one of the most commonly prescribed biomedical interventions, topical lidocaine. Pilot results showed significant pre- to post-treatment improvements in pain during sexual intercourse and sexual function for women with PVD, and sexual satisfaction for both women and partners. Pertinence, implications, challenges and limitations of conducting clinical research with this understudied and often-misunderstood pain population will be discussed.

\section{Session 106: 1:30 - 3:00pm}

\section{7}

\section{USING YOUTUBE VIDEOS AS A MEANS OF WIDELY DISSEMINATING KNOWLEDGE ABOUT PAIN}

\section{Chair: Denise Harrison}

Children's Hospital of Eastern Ontario, Ottawa, Ontario

Speakers: Karen Davis, Denise Harrison, Christine Chambers

Workshop Objective:

This symposium will focus on the use of the social media platform of YouTube as a means to disseminate pain research aimed at sharing knowledge about mechanisms of pain, and effective pain management during procedures in infants and children.

Learning Objectives:

1. To gain an understanding of methods used to disseminate pain research through the social medium of YouTube

2. Understand research methods used to track reach and impact of posted YouTube videos

3. Gain an understanding of the challenges and limitations of using social media as a knowledge translation medium

\section{A}

\section{HOW DOES YOUR BRAIN RESPOND TO PAIN? A TED-ED YOUTUBE ANIMATION}

\section{Karen Davis}

Toronto Western Research Institute (TWRI), Toronto, Ontario

TED-Ed (in partnership with YouTube) is a platform for educational animations and educational materials. The animations are produced jointly by an educator who creates a script and provides educational material, and a professional animator. As a Mayday Pain and Society Fellow, I developed a TED-Ed animation to convey two concepts of importance to scientists, clinicians, patients, the general public, and policy-makers: 1 . Pain, by definition, is a subjective experience and as such is best measured through self-report. This issue is important because of barriers from health care workers, insurance providers and the general public related to doubt as to the validity of intersubject differences in pain self-reports. 2. Brain imaging holds the potential to identify individual brain circuitry linked to pain perceptions and treatment response, and as such could provide a personalized approach to pain management; but should not be used to "decode" brain images for the purposes of validating self-report. The reach and impact of my video "How does your brain respond to pain?" far surpassed expectations with 400,000 views in the first week and over 510,000 in 6 months (50\% from unique viewers). TED-Ed analytics indicate that half the viewers were in the USA $(2-4 \%$ in each of Vietnam, Canada, UK, Saudi Arabia), mostly using a mobile phone $(40 \%)$ or computer $(40 \%)$ with the rest viewing on a tablet, game console, or TV. The video has been adopted as an educational tool by university educators and health care professionals in general practice, pain and physiotherapy clinics. 
7B

BE SWEET TO BABIES: USING YOUTUBE AS A MEANS TO DISSEMINATE EFFECTIVE PAIN MANAGEMENT STRATEGIES FOR INFANTS

Denise Harrison

Children's Hospital of Eastern Ontario, University of Ottawa, Ottawa, Ontario

The social media platform of YouTube was used, with the aim of showing parents ways to reduce needle-related pain in newborns and older infants, disseminating this knowledge to a far and wide audience and evaluating the reach and impact of the videos. Two parent-targeted videos were produced and posted showing; i) breastfeeding and sucrose during immunization in infants (posted October 2013); and ii) breastfeeding, skin-to-skin care and sucrose during bloodwork in newborns (posted July 2014). The neonatal pain video is currently available in English, French, Spanish and Portuguese and will soon be posted in Mandarin, Arabic and Inuktitut. YouTube statistics collected at 12 months following the release of the immunization video show 75,000 views. The top five countries viewing the video are: United States (22\%), Canada (14\%), Saudi Arabia (7\%), United Kingdom (4\%) and India (4\%). The average view duration is 1 minute 6 seconds (69\% of video). There were 86 likes, 17 dislikes and 40 comments. Dislikes were primarily related to anti-immunization. Only 162 surveys linked to the YouTube video were completed. This extremely low response rate $(0.24 \%)$ brings into question the usefulness of surveys linked to YouTube videos. For those who did complete the survey, the video had a positive impact on parents and health care professionals on intention to use breastfeeding or sucrose for subsequent immunizations. In this presentation, the challenges and future directions of evaluating the impact and effectiveness of social media forms of knowledge translation will be discussed.

\section{C}

IT DOESN'T HAVE TO HURT: DISSEMINATING EFFECTIVE STRATEGIES TO REDUCE IMMUNIZATION PAIN AND FEAR IN CHILDREN USING YOUTUBE

Christine Chambers

Dalhousie University, Halifax, Nova Scotia

In spite of significant research and clinical practice guidelines, the majority of children undergoing painful medical procedures such as immunization receive no pain relieving interventions. To address this gap, we developed a brief $(2 \mathrm{~min} 17 \mathrm{sec})$ YouTube Video for parents that summarized evidence-based strategies for pain management in a fun and entertaining way. In the video, a cute 4 year old girl tells parents what they should - and shouldn't - do to help make needles hurt less. The video was posted to YouTube on November 4, 2013 and received more than 26,000 views in the first two weeks and has continued to steadily gain additional views (current count $>46,000$ ). Data from preliminary analysis of video statistics collected by YouTube indicated that the video has been viewed in over 110 countries to date. $71 \%$ of viewers were female and $29 \%$ were male. The video was primarily watched from desktop ( $42 \%$ of views) and tablet ( $30 \%$ of views) computers. On-line surveys of parents and health professionals who viewed the video show strong acceptance of the video messages and intention to improve pediatric pain management behaviours after watching the video. This presentation will also summarize some of our successes and challenges using YouTube and other social media (e.g. Twitter) to share evidence-based information about children's pain.

\section{Session 107: 1:30 - 3:00pm}

\section{8}

\section{CANNABIS FOR PAIN: EVIDENCE, EDUCATION AND EVOLUTION}

Chair: Mark A Ware

Alan Edward Pain Management Unit, Montreal, Quebec

Speakers: Ruth Dubin, Mark A Ware, Mary Lynch

Workshop Objective:

Attendees will learn about the Marihuana for Medical Purposes Regulations (MMPR), its background and its foreseeable future; the history, development and future of the CFPC guidance documents, and recent systematic reviews of the clinical trial evidence for the use of cannabis and cannabinoids in the management of pain.

Learning Objectives:

1. Understand the history, rationale and mechanisms of the new Marijuana for Medical Purposes Regulations

2. Appreciate the challenges and concerns of the family physician regarding medical cannabis use and learn ways to manage these concerns.

3. Review and critique the evidence for the use of cannabinoid in the pain management of chronic non-cancer pain

\section{A}

\section{BLACK AND WHITE AND READ ALL OVER: THE COLLEGE OF FAMILY PHYSICIANS OF CANADA (CFPC) TACKLES THE ISSUE OF MEDICAL MARIJUANA \\ Ruth Dubin}

\section{Chronic Pain Committee}

With Health Canada's legislation allowing any physician to prescribe medical marijuana, the CFPC formed a working group to produce a guidance document for family physicians. Opinions around the use of medical cannabis are often polarized. Guided by Dr. Jamie Meuser, CFPC Executive Director, Professional Development and Practice Support, the chronic pain and addiction committees (with input from the Mental Health, Maternal and Newborn, Child and Adolescent, and Palliative Care Committees) produced a draft document with 12 recommendations, achieved by consensus. These recognized the lack of typical pharmaceutical product evidence, and also the need to balance risk with patient-centered care. Attendees at the 2014 Canadian Pain Society meeting provided valuable comments on the draft, which were incorporated into the final document. The CFPC has received comments from its members since the document was released and a revision is planned. This presentation will seek input from the audience of experts to better inform future versions of the document.

\section{B}

THE SAFETY OF MEDICAL CANNABIS: OLD APPROACHES AND NEW APPLICATIONS

\section{Mark A Ware}

Alan Edward Pain Management Unit, Montreal, Quebec

The implementation of a regulatory framework for medical cannabis has given rise to a number of concerns as the drug has not been approved by any standard drug review process. Among the concerns raised by clinicians is the lack of data on the long-term safety of cannabis when used for medical purposes. In many ways this situation is comparable with the long-term use of opioids, although in the case of opioids the concerns arose after approval of the drugs by standard regulatory procedures. It is clear that drug approval per se does not necessarily imply safety. This session will review drug safety study methodologies, and suggest methods to improve our understanding of the safety of long-term cannabis and cannabinoid use. Only through active clinician involvement will drug safety issues be identified and ideally prevented. 


\section{C}

\section{THE EFFICACY OF CANNABINOIDS FOR THE TREATMENT OF PAIN: RESULTS OF A SYSTEMATIC REVIEW}

\section{Mary Lynch}

Dalhousie University, Halifax, Nova Scotia

The prevalence of chronic pain is increasing as the population ages and as medical advances continue to improve survival following trauma or illnesses that previously would have been fatal. There is a growing cohort of survivors with serious chronic pain conditions. Currently available agents are inadequate to control all pain or are associated with limiting side effects and in this context, many people with chronic pain are turning to other therapies including cannabis. Due to patient demand, several nations, including Canada have developed programs to allow people with serious health conditions to access cannabis for medicinal purposes. Most of these programs (e.g. Canada, Israel, Netherlands, several US States) require physician or nurse practitioner support for the individual patient to be approved for access. Medical professionals need more information about the role of cannabinoids in pain management. A review of RCTs examining cannabinoids in the treatment of chronic non-cancer pain will be presented.

\section{Session 108: 1:30 - 3:00pm}

\section{9}

\section{HOT TOPICS}

Chair: Jeffrey S Mogil

Alan Edwards Centre for Pain Research, McGill University, Montreal, Quebec

Speakers: Samantha R Fashler, Neil A Hagen, Rebecca Price,

Alexander H Tuttle, Hichem Saidi, Mohammed F Shamji

\section{A}

\section{CANADIAN PAIN COALITION REPORT CARD ON PAIN: SYSTEMATIC REVIEW OF MULTIDISCIPLINARY CHRONIC PAIN SERVICES IN CANADA}

Samantha R Fashler ${ }^{1}$, L Cooper, LC Burns, S Razavi, L Goldberg, E Oosenbrug, J Katz

${ }^{1}$ York University, Toronto, Ontario

We reviewed the published literature evaluating multidisciplinary chronic pain centres across Canada to determine what services are available, what health care professional are practicing in clinics, wait times for access to services, and specific services offered. A systematic literature review was conducted using MEDLINE, PsycINFO, and CINAHL to identify articles that surveyed chronic pain services. The search yielded 1674 unique articles. Internationally, 12 satisfied our criteria with three surveying pain centres in Canada. Two articles reported on Canada-wide surveys and the third on a survey conducted in Québec. Surveys were conducted between 2002 and 2006 and published between 2005 and 2007. In total, 157 chronic pain clinics across Canada responded to the surveys. Mean clinic wait times ranged from four weeks to nine or more months. The vast majority of pain centres were located in urban centres $(61 \%-80 \%)$. The most commonly treated pain conditions included low back pain, neuropathic pain, headache pain, abdominal pain, complex regional pain syndrome, neck pain, and fibromyalgia. A wide variety of health care professionals worked in pain clinics; most prominently general practitioners, anaesthesiologists, and psychologists. Most clinics used a variety of interventional, physical, and psychological treatments. One survey determined that there was one pain clinic per 258,000 Canadians. Our systematic review identified three surveys of chronic pain centres in Canada published between 2005 and 2007. Prevalence rates show that approximately $20 \%$ of Canadians are living with chronic pain. Based on the survey data, we estimate there is only one pain clinic for every 51,600 sufferers. Since the most recent survey was published more than 8 years ago, updated information about professional composition, availability, and locations of chronic pain clinics across Canada is needed.

\section{B}

TETRODOTOXIN FOR MODERATE TO SEVERE CANCERRELATED PAIN: A MULTICENTRE, RANDOMIZED, DOUBLEBLIND, PLACEBO-CONTROLLED, PARALLEL-DESIGN TRIAL

Neil A Hagen ${ }^{1}$, L Cantin, J Constant, T Haller, G Blaise, M Ong-Lam, P du Souich, W Korz, B Lapointe

${ }^{1}$ University of Calgary, Calgary, Alberta

Cancer pain remains a serious public health issue: it can be difficult to control despite existing approaches to analgesia, and new treatments are needed. This study evaluated subcutaneous $\mathrm{Na}+$ channel blocker, tetrodotoxin (TTX) for the treatment of moderate to severe, inadequately controlled cancer-related pain. 165 subjects were enrolled in a Phase III trial at 19 sites in Canada, Australia and New Zealand. Unadjusted responder rate analysis supports a clinical benefit on the primary pain endpoint, significant at the one-sided $5 \%$ level $(p=0.0460)$ but not at the pre-specified two-sided 5\% level. After accounting for age, daily opioid dose and pain level, the primary pain endpoint produced an estimated responder rate difference of $23.1 \%$, nominal $\mathrm{p}=0.0127$ and $\mathrm{NNT}=4.3$. The Patient Global Impression of Change supported an analgesic benefit $(\mathrm{p} \leq 0.0003)$.

\section{C}

\section{OPIOID BLOCKADE DECREASES SUBJECTIVE RATINGS OF RELIEF}

Rebecca Price1, W Gandhi, M-E Hoeppli, S Becker, P Schweinhardt ${ }^{1}$ McGill University, Montreal, Quebec

Endogenous opioids' role in pain perception has received much attention and is well known, however their role in mediating pain relief has not been investigated. Thirteen healthy volunteers completed two testing sessions, one with placebo and one with the opioid antagonist, naltrexone. In each session, a $3 \times 3 \mathrm{~cm}$ area of $0.4 \%$ capsaicin cream was applied to the forearm. After $20 \mathrm{~min}$, the cream was removed and a $3 \times 3 \mathrm{~cm}$ thermode (Pathway, Medoc, Israel) was applied to the area. Using individualized temperatures, participants then adjusted the applied warm temperatures to maintain a constant sensation at two target intensities: 170 and 195. They then rated the unpleasantness and average intensity of the painful stimulation. Following the painful stimulation, the temperature decreased to $25^{\circ} \mathrm{C}$, during which time participants either rated relief or pleasantness. Participants completed 2 relief and 2 pleasantness trials at both 170 and 195. Data were analyzed using paired sample $t$-tests. There were no significant differences between unpleasantness ratings, average intensity ratings or applied temperatures during either 170 or 195 trials (all p's > 0.1). During 170 trials, naltrexone significantly decreased ratings of relief $(p=0.038)$ and pleasantness $(p=0.015)$ when the cool temperature was applied. Similarly, naltrexone significantly decreased ratings of relief $(p=0.004)$ and pleasantness $(p=0.007)$ during 195 trials when the cool temperature was applied. Despite a non-significant effect on pain, naltrexone decreased ratings of relief and pleasantness during a relieving stimulus.

\section{D}

\section{A META-REGRESSION ANALYSIS OF PLACEBO RESPONSE IN CLINICAL TRIALS OF NEUROPATHIC PAIN}

Alexander H Tuttle ${ }^{1}$, S Tohyama, T Ramsay, J Kimmelman, P Schweinhardt, GJ Bennett, JS Mogil

${ }^{1}$ McGill University, Montreal, Quebec

Increasingly, novel pharmacological targets for treating neuropathic pain are unable to pass randomized controlled drug trials, calling into question whether current drug study design may in part explain lack of demonstrable drug efficacy. In an effort to identify potential contributors to recent trial failures, we conducted a literature review of neuropathic pain placebocontrolled randomized clinical trials published between 1980 and 2014. After second-pass screening, a total of 80 articles were included in our final data set. Analysis of reported pain measures extracted from placebo and treatment groups yielded several striking correlations between study design and reported placebo-group pain scores. First, placebo ratings (reductions in pain measures) are becoming stronger over time. Furthermore, the increasing placebo response correlates with a significant decreasing trend in treatment advantage, indicating that increasing placebo response may 
negatively impact neuropathic pain trial outcome. Finally, the magnitude of the placebo response appears to be limited to North American studies. Taken together, these findings are similar to those reported in both the depression and schizophrenia literature. While the magnitude of the placebo response appears to be increasing over time, causal mechanisms of these trends are still unknown.

\section{E}

\section{LONG-TERM EFFECTIVENESS OF OPIOIDS AMONG CHRONIC NON-CANCER PAIN PATIENTS ATTENDING A MULTIDISCIPLINARY PAIN TREATMENT FACILITY: A QUEBEC PAIN REGISTRY STUDY}

Hichem Saidi ${ }^{1}$, G Pagé, M Ware, M Choinière

${ }^{1}$ Centre de recherche du centre hospitalier de l'universite de Montreal, Montreal, Quebec

The goal of the present study was to examine the long-term effectiveness of opioids in terms of reducing pain intensity (average and worst pain) pain interference and improving physical (pQofL) and mental (mQofL) healthrelated quality of life of patients with chronic non-cancer pain. Generalized Estimating Equations were used to examine whether opioid use profile (non-users, non-persistent users, and persistent users) predicted pain intensity, pain interference, as well as physical and mental health-related quality of life. Compared to non-users, persistent opioid users had significantly higher levels of worst pain intensity, average pain intensity and pain interference, and worse physical and mental health-related quality of life across time. Results suggest that persistent use of opioids does not improve painrelated outcomes in patients and further research is needed in order to examine diagnostic and prognostic factors that might contribute to this lack of treatment response.

\section{F}

\section{MECHANICAL ALLODYNIA FOLLOWING DISC HERNIATION REQUIRES INTRANEURAL MACROPHAGE INFILTRATION AND CAN BE STRATEGIES LIMITING MACROPHAGE ACTIVITY}

\section{Mohammed F Shamji ${ }^{1}$, YS Tu, MW Salter}

${ }^{1}$ Toronto Western Hospital, University of Toronto, Toronto, Ontario Disc-herniation radiculopathy arises from both mechanical compression and biochemical inflammation of apposed neural elements. The mechanisms giving rise to chronic pain in patients with non-compressive disc herniation remain unclear. This study demonstrates the role of inflammatory activation in generating a painful neuropathy phenotype after placement of heterotopic disc tissue onto rodent sciatic nerve. C57BL/6 mice underwent mid-thigh exposure of the sciatic nerve, with control animals exposed only and experimental animals having placement of littermate tail nucleus pulposus (NP) onto the nerve. Animals were evaluated throughout one week for mechanical allodynia, thermal hyperalgesia, cold allodynia, and gait stability. At sacrifice, immunohistochemistry was performed to identify perineural and intraneural macrophage and lymphocyte presence. The experiment was repeated under conditions of macrophage depletion or antagonized neurotrophin function. Mice exposed to NP stimulation demonstrated mechanical allodynia, thermal hyperalgesia, and cold allodynia compared with controls. Intraneural macrophage infiltration was observed in this group, alongside autoreactive lymphocytes at the disc-nerve interface. This behavioural phenotype was completely blocked with macrophage depletion or antagonized neurotrophin function. Non-compressive disc herniation generates pain behaviour in this animal disease model, alongside intraneural macrophage migration. Strategies to decrease perineural inflammation or maintain integrity of the blood-nerve-barrier may be effective in treating painful disc-herniation radiculopathy.

\section{Session 109: 4:00 - 5:30pm}

\section{0}

\section{ORGANIZATIONAL INITIATIVES TO INCREASE INTERPROFESSIONAL PAIN COMPETENCIES AND DECREASE THE KNOWLEDGE TO PRACTICE GAP}

Chair: Bonnie Stevens

Hospital for Sick Children, University of Toronto, Toronto, Ontario Speakers: Judy Watt-Watson, Bonnie Stevens, G Allen Finley

Workshop Objective:

To identify and describe organizational pain initiatives to increase interprofessional pain competencies and bridge the knowledge to practice gap. Learning Objectives:

1. To increase awareness and knowledge of interprofessional pain competencies.

2. To explore 3 examples of organizational initiatives to increase interprofessional pain competencies and decrease the knowledge to practice gap including:

a. An interfaculty pain curriculum in an academic setting;

b. A quality improvement plan to promote optimal pediatric pain assessment and management practices a clinical settings;

c. An international accreditation initiative for enhancing pain prevention and management.

3. To determine the impact of organizational context on outcomes.

\section{A}

\section{ENHANCING PAIN COMPETENCIES THROUGH AN} INTERPROFESSIONAL PAIN CURRICULUM

Judy Watt-Watson

University of Toronto, Toronto, Ontario

Although access to pain management has been declared a human right, unresolved pain remains a critical issue for individuals and health care professionals. This issue exists in part due to the lack of pain knowledge and skills. Surveys examining pain content in educational curricula reveal that health care professionals receive minimal education about pain. The goal to improve pain curricula and provide opportunities for interprofessional learning is critical if we are to change the continuing current ineffective pain practices documented worldwide. Moreover, the importance of interprofessional learning to encourage safe and effective collaborative patient care has been recognized by WHO and IASP. The IASP Interprofessional Pain Curriculum (IPC) (2012) was developed to provide a basis for different professions to learn a common understanding of pain assessment and management concepts. The IPC was based on the revised CORE and uniprofessional curricula and developed through an iterative process involving the interprofessional Education Initiatives Working Group. The process involved six phases with two years of discussion until consensus was reached on content relevant to all professions regardless of region. A subsequent Mayday funded consensus project has developed related core competencies to support the IPC domains. This presentation will briefly outline the IPC components and related competencies and describe how they have been addressed in a novel Pain Interprofessional Curriculum Design Workshop at the University of Toronto in 2014. Challenges in designing and implementing interprofessional pain curricula, and lessons learned for launching strategies to enhance pain competencies with interprofessional pain education will be presented.

\section{B}

\section{THE SICKKIDS PAIN CENTRE QIP INITIATIVE TO INCREASE PAIN COMPETENCIES AND IMPROVE CLINICAL PRACTICE OUTCOMES}

Bonnie Stevens

Hospital for Sick Children, University of Toronto, Toronto, Ontario The Hospital for Sick Children (SickKids) Pain Centre has developed and implemented a Quality Improvement Plan (QIP) in 15 inpatient units. The goal is to promote evidence-based pain assessment and 
management and improve clinical and satisfaction outcomes. The quarterly QIP includes (a) audit and feedback of pain assessment and management practices, implementation of knowledge translation (KT) strategies (e.g. reminders) and skill development (e.g. online pediatric pain education modules). A hospital-based audit tool captures the frequency of pain assessments, prevalence of severe to moderate pain, and the use of pain management strategies. Results are shared and recommendations are developed and communicated with unit staff using tailored KT strategies. Independently and externally collected NRCC-Picker satisfaction scores are compared across QIP units. Since the 2012 QIP launch, pain assessment documentation and pain management interventions for moderate to severe pain has increased. The proportion of patients with at least one pain assessment indicating moderate to severe pain decreased from $22 \%$ to $19 \%$ and similarly, the proportion of moderate to severe pain intensity assessments decreased from $10 \%$ to $9 \%$. The proportion of pain assessment completed one hour post-intervention (pharmacological or non-pharmacological) following an assessment of moderate to severe pain increased from $38 \%$ to $40 \%$. The percentage of patients and families responding positively to satisfaction questionnaire has increased from $75 \%$ to $83 \%$. Ongoing strategies to increase pain competencies and improve outcomes will be explored within the context of the SickKids organizational strategic plan. Challenges and future recommendations will be highlighted to changing practices using a QIP.

\section{$10 \mathrm{C}$}

\section{THE CHILDKIND INTERNATIONAL INITIATIVE -} AN ORGANIZATIONAL ACCREDITATION PROCESS FOR PAIN PREVENTION AND MANAGEMENT

\section{G Allen Finley}

\section{Dalhousie University, Halifax, Nova Scotia}

ChildKind International is a non-governmental organization aimed at reducing the pain and suffering of children around the world. The achievement of this aim involves implementation of standards on the effective prevention and treatment of pain in children at the institutional level. Research has shown that lasting change is more likely to occur when the desire for effective pain treatment is incorporated as a core institutional value. The ChildKind International strategy involves hospitals applying for and engaging in a multistep certification process. Certification is based on the completion of a comprehensive dossier that provides evidence of the institution's ability to meet 5 key principles, including leadership support, pain assessment and management, pain education, and the implementation of quality improvement processes. This certification process was piloted on four hospitals in the United States and Canada in 2013-14 and will be further developed through extension of this process to an additional 4-6 hospitals in 2014-15, with planned extension outside North America in following years. Challenges and strategies associated with improving pain competencies and decreasing pain in children will be discussed.

\section{Session 110: 4:00 - 5:30pm}

\section{1}

\section{TRAINEE SESSION: CAREER PATHS IN PAIN RESEARCH AND PRACTICE}

Chair: Whitney Scott

King's College London, London, United Kingdom

Speakers: Irene Tracey, Mark A Ware, Fernando Cervero

Workshop Objective:

Pain research trainees possess considerable skills to help them pursue a range of careers. The traditional academic faculty route is one path by which trainees may capitalize on their skills. Beyond academia, pain research trainees may pursue careers in areas as diverse as industry, clinical work, and patient advocacy. The purpose of this trainee oriented symposium is to highlight career opportunities available to basic and clinical pain research trainees for successfully deploying their many skills. The session will bring together a multidisciplinary group of experts who will reflect on their own careers paths in pain research and practice and provide advice to trainees for success across a range of careers. Active participation among attendees will be encouraged throughout the session.

Irene Tracey: Putting your skills to good use: Capitalizing on your transferable skills to succeed within academia and beyond

Mark A Ware: Diversifying and synergizing: Tips for developing and drawing on a range of skills to maximize your career success

Fernando Cervero: To boldly go: Blue skies research in a changing world Learning Objectives:

1. Become aware of transferable skills and talents that can be used to succeed in academia and a wider range of employment areas.

2. Identify strategies for successfully integrating research, clinical practice, and patient advocacy efforts.

3. Understand how the changing research climate may impact career development and the value of curiosity-driven research.

\section{Session 111: 4:00 - 5:30pm}

\section{2}

\section{NEURONAL EXCITABILITY AND PAIN}

Chair: Michael Hildebrand

Carleton University, Ottawa, Ontario

Speakers: Jason McDougall, Slobodan Todorovic,

\section{Michael Hildebrand}

Workshop Objective:

This session will highlight current research aimed at identifying the specific types of receptors and channels which play critical roles in shaping the excitability of nociceptors and dorsal horn neurons. We will discuss the molecular mechanisms whereby changes in receptor/channel activity result in increased neuronal activity and pathological pain. Finally, we will offer insights into how this knowledge could potentially lead to new molecular targets for the treatment of pain.

Learning Objectives:

1. To gain an appreciation of the diverse types of receptors and channels that regulate the excitability of peripheral nociceptors and central dorsal horn neurons, including proteinase-activated receptors, voltage-gated calcium channels, and synaptic NMDA receptors.

2. To understand how enhanced activation of these receptors and channels leads to neuronal hyperexcitability in models of inflammatory and neuropathic pain.

3. To gain insights into potential new therapeutic strategies for reversing pathological pain by targeting specific modulators of channel/ receptor activity.

\section{A}

\section{PAIN SIGNALLING BY PROTEOLYTIC ENZYMES}

\section{Jason McDougall}

Dalhousie University, Halifax, Nova Scotia

During inflammation, proteolytic enzymes are released into damaged tissues to assist with healing and vascular management. It has recently been discovered that these enzymes can also act as pain signalling molecules by cleaving a distinct family of $\mathrm{G}$ protein-coupled receptors called proteinase activated receptors (PARs). This presentation will highlight the neurophysiological mechanisms involved in PAR responses by focussing on proteinase-induced pain regulation in arthritic joints.

\section{B}

REGULATION OF NEURONAL EXCITABILITY OF PERIPHERAL NOCICEPTORS VIA POST-TRANSLATIONAL MODIFICATION OF VOLTAGE-GATED CALCIUM CHANNELS

\section{Slobodan Todorovic}

University of Virginia School of Medicine, Charlottesville, Virginia, USA

Voltage-gated calcium channels are ubiquitously expressed in neurons and are key regulators of cellular excitability and synaptic transmitter release. Several studies have established that both T-type and highvoltage-activated (HVA) subtypes of voltage-gated calcium channel can 
be redox-regulated. In this presentation I will discuss covalent posttranslational modification via oxidation of cysteine molecules and chelation of trace metals, as well as reactions involving nitric oxide-related molecules and free radicals. Improved understanding of the roles of redox-based reactions in regulation of voltage-gated calcium channels may lead to improved understanding of novel redox mechanisms in physiological and pathological processes. Identification of redox mechanisms and sites on voltagegated calcium channel may allow development of novel and specific ion channel therapies for unmet medical needs. Thus, it may be possible to regulate the redox state of these channels in treatment of pathological process such as neuropathic pain.

\section{$12 \mathrm{C}$}

SYNAPTIC NMDA RECEPTORS IN SPINAL MECHANISMS OF PAIN PROCESSING

Michael Hildebrand

Carleton University, Ottawa, Ontario

Lamina I neurons in the dorsal horn of the spinal cord are a hub for sensory integration and transmission within the pain processing network. Pain hypersensitivity depends on the activation and potentiation of NMDA receptors (NMDARs), yet, a major open question is where is the critical locus for this change in NMDAR activity within the pain signalling pathway? This presentation will describe specific electrophysiological approaches used to characterize for the first time the subtypes of NMDA receptors that mediate direct synaptic responses in lamina I spinal neurons. A novel mechanism of hyperexcitability will then be highlighted which couples the loss of inhibition to facilitation of NMDARs at lamina I synapses during pathological pain processing.

\section{Session 112: 4:00 - 5:30pm}

\section{3}

\section{INTERVENTIONAL MANAGEMENT OF PAIN}

\section{Chair: Mohammed F Shamji}

Toronto Western Hospital, University of Toronto, Toronto, Ontario Speakers: Anuj Bhatia, Philip Peng, Mohammed F Shamji

Workshop Objective:

Proper application of interventional techniques in the management of chronic pain has the potential to affect pain, disability, quality of life, and pharmacotherapy use. This session will instruct the audience about the evidence, techniques, and outcomes of various interventional options for the management of chronic neuropathic pain.

Learning Objectives:

1. To review the evidence and techniques for injection procedures in the management of chronic pain

2. To discuss the evidence for drug infusions in the management of neuropathic pain

3. To define the indications and review techniques for spinal cord stimulation in the management of neuropathic pain

\section{A}

\section{INTERVENTIONAL PROCEDURES FOR TREATMENT OF CHRONIC PAIN}

\section{Anuj Bhatia}

\section{Toronto Western Hospital, Toronto, Ontario}

Interventional procedures for treatment of chronic non-cancer pain are often used both independently and within the context of multimodal therapy. Apart from procedures involving implantable therapies (neurostimulation and intrathecal pumps), techniques for relieving chronic pain can be classified as neuraxial and peripheral. Neuraxial procedures include injections of local anesthetics with or without steroids in the epidural space for degenerative spine disease and in arthritic facet and sacroiliac (SI) joints, diagnostic and therapeutic selective nerve root blocks for herniated intervertebral discs compressing spinal nerve roots, diagnostic and definitive (radiofrequency ablation) procedures involving nerve supply to facet and SI joints. Peripheral procedures include injections of local anesthetics with or without steroids around injured peripheral nerves and into major and minor arthritic limb joints. Current evidence on efficacy and effectiveness of these procedures will be presented at the symposium and recommendations based on this evidence will be discussed. Limitations of available evidence and controversies regarding recommendations on use of these procedures will be also presented.

\section{B}

\section{INFUSION TECHNIQUES FOR NEUROPATHIC PAIN Philip Peng} Toronto Western Hospital, Toronto, Ontario

Neuropathic pain results in severe impact in patient quality of life. Various drug infusion methods have been used including the use of ketamine and lidocaine infusions which attracts substantial interest in the medical community. This presentation will review and discuss the philosophy of the use of these infusion methods, and the evidence of the outcome following those treatments.

\section{$13 \mathrm{C}$}

\section{SPINAL CORD STIMULATION FOR NEUROPATHIC PAIN}

\section{Mohammed F Shamji}

Toronto Western Hospital, University of Toronto, Toronto, Ontario

The management of extremity neuropathic pain can be challenging with treatments ranging from psychotherapeutic to pharmacological to neuromodulation. It is incumbent on physicians managing chronic pain to be familiar with the array of potential interventions. With randomized controlled trials and technological innovation, the utility of spinal cord stimulation (SCS) will continue to grow with expanding indications continuing to be demonstrated. The SCS technique has been rigorously evaluated and compared to medical management for a variety of pain diagnoses in which it has been found to reduce chronic pain and disability in a clinical and cost effective fashion. This presentation will review mechanism, evidence, and techniques of SCS for the management of treatment-refractory neuropathic pain. Future avenues of investigation including novel pain indications and potential for motor recovery in spinal cord injury will also be introduced.

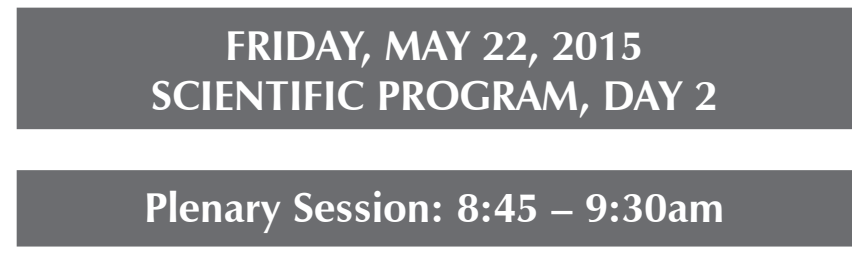

\section{5}

\section{A LIPID KINASE THAT REGULATES NOCICEPTIVE SIGNALING AND SENSITIZATION \\ Speaker: Mark Zylka}

Associate Professor, Department of Cell Biology \& Physiology, UNC Neuroscience Center, University of North Carolina, Chapel Hill, North Carolina, USA

Numerous pain-producing (pronociceptive) receptors signal via phosphatidylinositol 4,5-bisphosphate (PIP2) hydrolysis. However, it is currently unknown which lipid kinases generate PIP2 in nociceptive dorsal root ganglia (DRG) neurons and if these kinases regulate pronociceptive receptor signaling. In this presentation, I will describe a lipid kinase that is expressed in all DRG neurons, including peptidergic and nonpeptidergic nociceptive neurons, and that generates at least half of all PIP2 in DRG neurons. A genetic deficiency of this kinase leads to reduced pronociceptive receptor signaling in DRG neurons and reduced noxious thermal and mechanical sensitization in mouse models of chronic pain. This kinase also regulates signaling downstream of the capsaicin receptor TRPV1. We identified a novel small molecule inhibitor of this kinase in a high-throughput screen. This inhibitor lowered PIP2 levels in DRG neurons and attenuated noxious thermal and mechanical sensitization when administered intrathecally. Our studies reveal that this lipid 
kinase regulates PIP2-dependent nociceptive signaling and suggest that this lipid kinase is a novel therapeutic target for chronic pain.

Learning Objectives:

1. Educate audience about a lipid kinase that has not previously been studied in nociceptive neurons.

2. Provide new information about how this kinase regulates phosphoinositide levels and receptor signaling in nociceptive neurons

3. Audience will also learn about how to identify inhibitors of this kinase using a novel high throughput screen we developed.

\section{Early Career Award Lecture: 9:30am - 10:00am}

\section{6}

MECHANISMS, OUTCOMES, AND MOBILIZATION OF MATERNALLY-LED INTERVENTIONS FOR NEWBORN CARE

Speaker: Marsha Campbell-Yeo

Dalhousie University, Departments of Pediatrics and Psychology \&

Neuroscience, Halifax, Nova Scotia

\section{Session 201: 11:00am - 12:30pm}

\section{7}

PROBING NEURAL NETWORKS DYNAMICS OF PAIN AND ATTENTION: INSIGHTS INTO BRAIN MECHANISMS UNDERLYING INDIVIDUAL VARIABILITY IN PAIN COPING AND CHRONIC PAIN VULNERABILITY

\section{Chair: Karen Davis}

Toronto Western Research Institute (TWRI), Toronto, Ontario Speakers: Aaron Kucyi, Etienne Vachon-Presseau, Mathieu Roy Workshop Objective:

This Symposium, chaired by Karen Davis, will examine how neural networks represent individual differences in pain perception, attention, associated mood changes, and pain avoidance. Data from cutting edge imaging and modeling approaches, including dynamic functional connectivity, tractography, and graph theory, will shed light on the dynamic states of these representations, and how these may reflect pain sensitivities, coping, vulnerabilities and transitions to chronic pain states.

Learning Objectives:

1. Understand the new, emerging theories of network organization and dynamics underlying acute and chronic pain.

2. Understand how network dynamics variability represent potential sources of intersubject pain variability and vulnerabilities, and conversely how chronic pain may impact neural networks of pain.

3. Understand concepts of pain motivation and learning models that underlie pain avoidance choices.

\section{A}

\section{BRAIN DYNAMICS OF INTRINSIC ATTENTION TO PAIN Aaron Kucyi \\ Harvard Medical School, Boston, Massachusetts, USA}

To date, pain-attention interactions and their neural representation have largely been studied with approaches that involve explicit manipulation of pain. However, brain network communication and attentional states can be highly dynamic, both spontaneously and in the presence of sensory stimuli evoking pain. We recently introduced a new paradigm and neuroimaging analysis approaches to capture the brain dynamics of spontaneous attentional fluctuations during painful stimulation. We identified brain networks, including attentional and antinociceptive systems, that are engaged during spontaneous fluctuations in attention ("mindwandering") away from pain. This talk will describe our approaches, their relevance for understanding acute and chronic pain as well as individual variability in tendencies to attend to pain. An emphasis will be placed on how dynamic functional connectivity analysis, a novel approach gaining popularity in the neuroimaging community, can inform our understanding of pain-attention interactions. Implications for development of personalized pain therapies and for our understanding of a "neural signature" of pain will be discussed.

\section{B}

\section{A NEUROLOGICAL MODEL OF VULNERABILITY FOR CHRONIC PAIN}

Etienne Vachon-Presseau

Northwestern University, Evanston, Illinois, USA

The neurophysiological mechanisms of chronic pain are currently poorly understood. Here, we combined measures of genetic imprinting, morphology, structural and functional connectivity in subacute back pain patients that were followed longitudinally over a period of $1-3$ years as they recover or transition to persisting pain state. The brain was first studied as a network to show that stronger structural connections in the limbic brain initially predisposed to chronic pain. Graphs were secondly constructed from nodes delineating a fined-grained representation of the limbic system to show that stronger structural and functional connections in the corticostriatal module represented risk factors for chronic pain. Patients transitioning to chronic pain also displayed about $10 \%$ smaller hippocampal and amygdala volumes when compared to individuals recovering from their pain and healthy controls. No time effect interacted with the group, indicating the absence of neurotoxic effects of persistent pain on these functional, structural and morphometric measures. Alleles of the Opioid receptor delta 1 rs678849 were further associated with smaller amygdala volume and the transition to chronic pain. Structural equation modelling finally revealed that structural connectivity of the corticostriatal system and the right amygdala volume mediated the relations between mood, genetics and persistent pain. These results have important implications because they reveal a series of measures of limbic brain disruption and demonstrate that these elements were hard-wired before pain transition to chronic states. Better understanding these "emotional" predispositions for chronic pain and disability may lead to new treatment for preventing the transition to chronic pain.

\section{C}

\section{CEREBRAL MECHANISMS OF PAIN AVOIDANCE LEARNING}

\section{Mathieu Roy}

\section{Concordia University, Montreal, Quebec}

Pain is a primary driver of learning and motivated action. In order to study the brain mechanisms underlying pain's motivational function, we conducted a functional magnetic resonance (fMRI) study during which participants $(n=26)$ performed an instrumental pain avoidance. Using a combination of computational reinforcement-learning (RL) models, axiomatic tests, and dynamic causal models (DCM) models, we identified a candidate system that allows humans to avoid choices associated with pain. In this model, a system of interconnected forebrain regions including the putamen, hippocampus and ventromedial prefrontal cortex (vmPFC) encodes expected value signals. Expectations are then compared with primary nociceptive inputs in the periaqueductal gray (PAG) to generate the aversive prediction error PE signals critical for learning to avoid pain. Moreover, switches in choices following painful stimulations were not only predicted by RL model-based estimated probability of switching, but also by subjective pain ratings, suggesting two routes for pain's effects on behavior: 1 ) a model-based route where choices are performed by comparing the values of possible actions, and 2) a pavlovian route where avoidance is directly related to subjective pain intensity. Participants who relied more on pain ratings to perform their choices were less successful at avoiding pain, suggesting that pain's pavlovian effects on behavior can lead to sub-optimal decisions. The results of the present study are an important first step in providing a mechanistic account of the neural circuits underlying pain-related learning and motivation, with important implications for our comprehension of both aversive learning and chronic pain conditions. 
Session 202: 11:00am - 12:30pm

18

\section{KEEPING THE "I" IN PAIN: NOVEL APPROACHES FOR INTEGRATING THE SUBJECTIVE PAIN EXPERIENCE INTO THEORY, RESEARCH AND PRACTICE}

Chair: Timothy H Wideman

McGill University, Montreal, Quebec

Speakers: Timothy H Wideman, Geoff Bostick, Whitney Scott

Workshop Objective:

Participants will be able to better conceptualize and address the subjective experience of pain within research and clinical settings.

Learning Objectives:

1. Participants will be able to use the Measurement Model of Pain as a conceptual framework for relating first and third-person perspectives of pain within research and clinical practice settings.

2. Participants will understand how established approaches to mixedmethodological research can help clinicians and researchers better evaluate others' pain by integrating narrative reports of the subjective pain experience with data from quantitative pain measures.

3. Participants will have a better understanding of the experience and potential impact of invalidation in patients with pain, and will learn practical strategies for incorporating patients' subjective pain experience into the clinical encounter.

\section{A}

CONCEPTUALIZING THE "I" AND "YOU" OF PAIN: INTRODUCING THE NOVEL MEASUREMENT MODEL OF PAIN AS A MEANS OF RELATING FIRST AND THIRDPERSON PERSPECTIVES OF PAIN

\section{Timothy H Wideman}

McGill University, Montreal, Quebec

For centuries, philosophers have struggled to reconcile the first-person experience of pain with its third-person observation; this challenge continues to confront all researchers and clinicians attempting to measure or manage another person's pain. Current scientific debates address this issue by questioning the unique value of self-reported pain, the identification of gold standard pain measures, the validity of pain "biomarkers", and the conceptualization of chronic pain as a brain disease. This presentation will make the argument that reconsidering a historical perspective on pain specifically René Descartes' - will help chart a path forward in resolving these issues. Drawing on a revised view of Descartes, the presenter advances a novel Measurement Model of Pain (MMP) that classifies the personal experience of pain from a third-person perspective. The MMP includes the following postulates: The first-person experience of pain cannot be directly observed or measured from the third-person perspective; From the third-person perspective, the qualitative pain narrative is the best available proxy for this non-observable experience; The best approach to pain measurement is through a collection of multi-modal and multidimensional measures that are grounded to the personal pain narrative. Building on these principles, the presenter will outline a framework for how first and third-person perspectives on pain can be integrated in both research and practice.

\section{B}

LET'S MEET IN THE MIDDLE: INTEGRATING PATIENT NARRATIVES AND QUANTITATIVE MEASURES FOR A BETTER UNDERSTANDING OF THE PAIN EXPERIENCE

\section{Geoff Bostick}

University of Alberta, Edmonton, Alberta

The experience of pain is subjective and personal. However, recommendations for the assessment of pain in research and clinical settings tend to favour soliciting quantitative data in the form of pain ratings, survey scores and quantitative sensory testing responses. These data are researcher/clinician driven (i.e. from a third-person perspective), meaning the data acquired may not include information the sufferer wants to relate, and fails to capture the unique contextual aspects of the subjective pain experience. Qualitative data (e.g. pain narratives) capture these rich contextual perspectives, but by definition cannot objectively measure change in the pain experience. Both forms of data have clear value, however the nature of these data are so different (from both philosophical and practical perspectives) that we rarely see them purposefully integrated. This presentation aims to reconcile the disparity between quantitative and qualitative paradigms to inform pain measurement strategies in both research and clinical practice. This presentation will briefly describe a philosophical orientation (Pragmatism) that is in alignment with contemporary perspectives on pain measurement. Subsequently, established approaches to mixed-methods research design commonly used in other fields will be describe to demonstrate the potential benefits of combining qualitative and quantitative data for measuring pain. Finally, mixed-methods literature will be used to illustrate a potential approach to clinical reasoning that can be applied in education settings to improve student and clinician understanding of pain measurement.

\section{$18 \mathrm{C}$ \\ INTEGRATING THE SUBJECTIVE EXPERIENCE OF PAIN INTO CLINICAL PRACTICE FROM THE PERSPECTIVE OF A PAIN PSYCHOLOGIST \\ Whitney Scott}

King's College London, London, United Kingdom

Adequately assessing the subjective pain experience is considered fundamental in the clinical management of pain. Despite this, some patients with chronic pain report feeling that their pain problem is not understood by health care professionals. In light of this issue, there is growing interest in understanding the experience of invalidation in patients with chronic pain. Invalidation describes rejection, disbelief, or a lack of understanding from the social environment in response to one's expression of pain. Recent research on perceived injustice in the context of pain suggests that, beyond pain itself, individuals may feel invalidated with respect to the totality of suffering and loss associated with pain. Emerging research indicates that invalidation and related constructs, such as perceived injustice, contribute to adverse outcomes, including increased pain, disability, depression, poorer interpersonal relationships, and poorer recovery following multidisciplinary rehabilitation. This presentation will examine the problem of invalidation in patients with pain and will provide practical strategies that may be used in the clinical encounter to address this issue. Data examining the phenomenology and negative impact of invalidation and related constructs will first be presented. Next, the presenter will discuss clinical strategies, including whole-person assessment and validation techniques, which might facilitate greater understanding of the subjective pain experience and strengthen the therapeutic alliance. Finally, examples of how to incorporate clinical knowledge of the subjective pain experience into psychotherapeutic interventions for pain management, such as relaxation and hypnosis, will be explored.

\section{Session 203: 11:00am - 12:30pm}

\section{9}

EVALUATION OF ABUSE DETERRENT OPIOIDS IN THE OPIOID-DEPENDENT POPULATION: AN OVERVIEW OF CLINICAL APPROACHES, ETHICAL CONSIDERATIONS, AND PATIENT MANAGEMENT CHALLENGES

Chair: Beatrice Setnik

INC Research

Speakers: Beatrice Setnik, Pierre Geoffroy, Jack Corman

Workshop Objective:

This symposium will discuss the importance of including high opioiddependent or methadone-maintained subjects in opioid development, the clinical challenges, ethical considerations, and study methodology required to accommodate this population and the clinical approaches to mitigating risk and ensuring subject safety during this evaluation. 
Learning Objectives:

1. To provide an overview of the methodological approaches for clinical trials assessing abuse deterrent opioids in methadone-maintained patients

2. To explore how lessons learned from managing methadone-maintained patients can help identify safe and effective strategies in treating patients with co-morbid substance use disorder

3. To examine relevant guiding ethical principles that may justify research in opioid-dependent populations

\section{A}

ASSESSMENT OF OBJECTIVES AND METHODOLOGICAL APPROACHES FOR ASSESSING ABUSE DETERRENT OPIOIDS IN DEPENDENT AND METHADONE-MAINTAINED PATIENTS

Beatrice Setnik

\section{INC Research}

In December 2014, Health Canada issued a new guidance for industry addressing the development and submission of tamper-resistant formulations of opioids. Such formulations are designed to resist a number of common tampering methods and potentially provide barriers to certain methods of abuse. As opioid abuse is an important public health and safety issue, the rigorous assessment of products with tamper-resistant properties is integral in determining whether these products are able to reliably mitigate the potential for abuse. The guidance refers to a battery of assessment that includes human abuse potential studies. Such studies are typically conducted, as per guidances from both Health Canada and the FDA, in healthy, non-dependent recreational drug users. Whilst these studies are effective in assessing abuse potential in this population, the non-dependent abuser represents only a subset of the drug abusing population. The dependent abuser, which may be a more vulnerable user often prone to more risk taking behavior, is often overlooked in this evaluation. In recent years, an increasing number of studies have been including this relevant population, including methadone-maintained patients, in the assessment of abuse potential. The use of dependent populations in clinical trials needs to be carefully considered to ensure safety and ethical integrity. In addition, the medical management of withdrawal symptoms needs to be carefully implemented. The relevance of this population, as well as the clinical considerations and methodological adaptations that need to be considered will be examined during this session. Relevant study endpoints, including withdrawal assessment scales will be discussed.

\section{$19 B$}

\section{A REVIEW OF SAFE AND EFFECTIVE STRATEGIES WHEN TREATING PAIN PATIENTS WITH CO-MORBID SUBSTANCE USE: LESSONS LEARNED FROM THE MANAGEMENT OF METHADONE-MAINTAINED PATIENTS}

\section{Pierre Geoffroy}

\section{First Step Medical Clinic, INC Research}

Approximately, 90,000 Canadians suffer from an opioid addiction and over 200,000 Canadians may be abusing. Opioid replacement therapy (ORT) with Buprenorphine or methadone is an effective treatment for this substance use disorder. Usually prescribed within the confines of a contingency treatment plan, ORT allows for no use of opioids during treatment without penalties. Individuals on ORT are often stigmatized in the clinical setting. Too many family physicians avoid enrolling patients into their practice who suffer from opioid dependence. Consequently, the patient on ORT who is suffering from a painful ailment is often forced to walk-in clinics or hospital emergency rooms where she may be seen as drug seeking. Patients with a substance use disorder are as likely as anyone to suffer from painful conditions. This is equally true for patients on ORT. While buprenorphine and methadone afford good analgesic effects, the dose given, as well as their pharmacology, may be insufficient in treating a recovering addict's pain and, as such, additional analgesics may be necessary. This session aims to demystify the treatment of recovering addicts suffering from painful conditions. A review of opioid substance use disorder classification using DSM V will be followed by a brief discussion of ORT and its principle concepts. Following this, useful and pragmatic techniques for treatment of the opioid addict in pain will be discussed.

\section{C}

\section{ABUSE DETERRENT OPIOIDS IN OPIOID-DEPENDENT POPULATION: WHAT MAKES RESEARCH ETHICAL?}

\section{Jack Corman}

IRB Services (A Chesapeake IRB Company)

The Canadian Tri-Council Policy Statement 2ed establishes the broad framework for the ethical review and conduct of research involving humans. The TCPS2 core principles upon which this framework is based are Respect for Persons, Concern for Welfare, and Justice. These principles are highly convergent with the Belmont Report, upon which the US regulatory frameworks for both industry sponsored and US federally funded clinical research are based. Human research with opioids has evolved into an increasingly complex and challenging endeavor as data requirements from regulators, clinicians and payers have moved beyond classic drug development testing to demanding highly specialized research designs involving a population of experienced opioid users as subjects who may be particularly vulnerable to undue influence and more likely to suffer harms from the research than patients or normal healthy volunteers. It is ethically permissible to use vulnerable subjects in clinical research, but only under certain circumstances and with special considerations. This presentation will briefly explore the application of these general core ethical principles to the specific situations faced by researchers working in this field in order to confer adequate human subject protections to ensure ethical conduct of these research studies.

\section{Session 204: 11:00am - 12:30pm}

\section{0}

"ALL MY JOINTS HURT AND PEOPLE THINK I'M CRAZY!" PAIN AND HYPERMOBILITY IN YOUNG PEOPLE

Chair: G Allen Finley

Dalhousie University, IWK Health Centre, Halifax, Nova Scotia Speakers: Anthony Vandersteen, Karl J Logan, Michael Sangster Workshop Objective:

We hope to provide a logical approach to assessment and management of children and adolescents with both chronic pain and hypermobility. There will be ample opportunity for audience discussion and sharing of experience. Learning Objectives:

1. Attendees will understand the implications of hypermobility and genetic diagnoses in relation to chronic pain in children and adolescents.

2. Attendees will be aware of current orthopaedic questions related to hypermobility and pain, as well as appropriate surgical and non-surgical management approaches.

3. Attendees will be able to articulate the current best evidence physiotherapy management of patients experiencing neuropathic pain associated with joint hypermobility syndrome. Attendees will be able to articulate the current understanding of pathophysiology of the development and maintenance of neuropathic pain associated with joint hypermobility syndrome.

\section{A}

“ALL MY JOINTS HURT, AND IT'S MY PARENTS' FAULT" Anthony Vandersteen

Dalhousie University, IWK Health Centre, Halifax, Nova Scotia Joint hypermobility is a common trait in the population; we will present current epidemiological evidence showing an association between joint hypermobility and chronic musculoskeletal pain, dysautonomia, and chronic pain syndromes. We will review the prevalence of joint hypermobility in patients with functional bowel disorders, dysautonomia and anxiety disorders. We will describe pain symptoms and prevalence in patients with a variety of rare hereditary disorders of connective tissue (HDCTs) of known cause: Ehlers-Danlos Syndrome classical type, Marfan syndrome, tricho-rhino-phalangeal syndrome, Filamin A periventricular nodular heterotopia and Loeys Dietz syndrome. We will describe the multi-system burden of disease in these patients (vascular, cardiac, ocular, musculoskeletal, neurological). We will review the clinical presentation and diagnostic criteria for the most common HDCT: 
Ehlers-Danlos Syndrome hypermobility type / Joint hypermobility syndrome, for which the genetic basis remains largely unknown. The natural history, pain symptoms and multi-systemic burden of disease in children and adults will be presented. There are startling differences in approach by clinicians when assessing patients with known or unknown genetic cause. We describe a robust diagnostic approach and the dilemmas of disease labeling for these patients. The overlap in symptoms and diagnostic criteria with chronic fatigue syndrome are presented. We explain the resulting hurdles for patients with these diagnoses in accessing care for their multiple pathologies, the role of patient support groups and possible avenues for research as a result of a deeper understanding or molecular pathology.

\section{B}

\section{"ALL MY JOINTS HURT, AND MY DOCTOR THINKS I'M CRAZY" \\ Karl J Logan}

Dalhousie University, IWK Health Centre, Halifax, Nova Scotia

Orthopaedic surgeons face considerable challenges when children with pain and joint problems present. Ligamentous laxity is ubiquitous in children presenting to paediatric orthopaedic services. Painless flexible flat feet, for instance, can be seen in approximately $50 \%$ of children and $25 \%$ of adults. Within this large group of patients are those who have musculoskeletal pain related to their ligamentous laxity, some of whom will have joint hypermobility syndrome and a smaller proportion who will have a clearly diagnosed hereditary disorder of connective tissue. This group of patients have historically been neglected by the medical community as the search for organic pathology to explain their symptoms with current imaging techniques is limited. Ligamentous laxity can also lead to joint instability, dislocation, and skeletal deformity such as scoliosis. Surgery in this population should be performed reluctantly, and after exhaustion of nonoperative modalities of treatment, as successful outcomes are much less predictable. We will explore the patient's perspective, the presentation of these patients to the orthopaedic community, and conclude with a case based discussion of the non-operative and operative management of the orthopaedic manifestations of these conditions.

\section{C}

"ALL MY JOINTS HURT, AND IT'S GETTING ON MY NERVES" Michael Sangster

Dalhousie University, IWK Health Centre, Halifax, Nova Scotia

Chronic widespread pain is highly prevalent and severe in connective tissue disorders such as Ehlers-Danlos Syndrome. Pain severity appears to be correlated with hypermobility and contributes to overall functional impairment. The musculoskeletal complaints attributed to joint hypermobility syndrome are often refractory to conventional physical therapy intervention. The hypothesized pathophysiological mechanisms for the development and maintenance of chronic pain associated with joint hypermobility syndrome, including stretch injury to nerves traversing hypermobile joints, increased fragility of nerve connective tissue, and nerve trauma will be presented. The clinical presentation of the patient with peripheral nerve hypermobility mechanosensitivity will discussed and the clinical criteria used in physical therapy diagnosis, namely clinical neurodynamics, will provided. Novel physical therapy management guidelines will be considered in the context of a peripheral and central mechanisms-based understanding of the hypermobile patient with pain. Techniques to modify the patient's cognitive evaluation of the pain experience via therapeutic neuroscience education and cognition targeted exercise therapy as a strategy to reduce pain, disability, anxiety, stress, and improve endogenous pain inhibition will be critically reviewed. The evidence for movement based therapy in the management of neuropathic pain associated with joint syndrome, namely neurodynamic mobilization, will be presented.

\section{Session 205: 1:30 - 3:00pm}

\section{1}

\section{A NOVEL, MULTIDISCIPLINARY, TRANSITIONAL PAIN SERVICE DESIGNED TO MANAGE SEVERE, ACUTE POSTSURGICAL PAIN AND PREVENT THE TRANSITION TO CHRONICITY}

Chair: Joel Katz

York University, Toronto, Ontario

Speakers: Salima Ladak, Hance Clarke, Aliza Weinrib

Workshop Objective:

This symposium will discuss the clinical criteria that were developed to identify patients - during the early post-surgical period - who would benefit from inter-professional care from the TPS. Furthermore, the symposium will utilize cases form the TPS to illustrate the novel psychological approach to managing acute and persistent pain after surgery and will present both pharmacological and non-pharmacological management techniques employed by the pain specialists with a focus on the successes and challenges of implementing the TPS over the past year.

Learning Objectives:

1. Symposium participants will understand recent trends in the prevalence of persistent post-surgical pain.

2. Participants will understand the multidisciplinary team involved in the Transitional Pain Service.

3. Participants will understand the role of the psychologist in this novel program to prevent persistent pain after surgery.

\section{A}

\section{WHO IS AT RISK FOR CHRONIC POST-SURGICAL PAIN? EXPLORING THE PRE AND POST-OPERATIVE PERIOD}

\section{Salima Ladak}

University Health Network, University of Toronto, Toronto, Ontario It is widely accepted that unmanaged acute post-surgical pain may transition to a persistent pain problem, resulting in a negative impact on the patient's and family's quality of life. While analgesics are prescribed for the postdischarge period, some patients continue to experience unmanaged pain, and may not have timely access to an interprofessional team for the sole purpose of managing pain. This often results in patients seeking pain management treatment from an emergency department or unscheduled visitations with community medical clinics. The TGH Transitional Pain Service has found that the first few post-surgical days are an essential time to identify patients who are at high risk for developing severe pain and continue to require an integrated and personalized pain management plan from their health care providers. Structured pain management interventions following hospital discharge could reduce the risk of developing persistent pain. This presentation will discuss in-depth the clinical criteria that were developed to identify patients - during the early post-surgical period - who would benefit from interprofessional care in the transitional pain clinic.

\section{B \\ THE IMPLEMENTATION OF THE TRANSITIONAL PAIN SERVICE: SUCCESSES AND CHALLENGES}

\section{Hance Clarke}

University of Toronto, University Health Network, Toronto, Ontario Post-surgical chronic pain accounts for one quarter of patients presenting to chronic pain clinics. Patients who develop chronic post-surgical pain are at increased risk for persistent opioid use. Ontario data demonstrates that $50 \%$ of patients are discharged with opioid analgesics following major surgery and $3 \%$ of previously opioid-naïve patients continue to take these medications 6 months later. Significant gaps in the continuity of care after major surgery are responsible for unrelieved pain, hospital re-admissions, and ongoing opioid use among complex pain patients discharged without appropriate follow-up plans or care, and without pain specialists able to manage their postsurgical pain and/or successfully wean them from opioid medications. We describe the development and implementation of a novel 
multidisciplinary Transitional Pain Service (TPS) comprising a team of pain specialists including, nurse practitioners, anesthesiologists, psychologists, physiotherapists, acupuncturists, and family physicians. The TPS is designed to improve in-hospital pain care, reduce hospital length of stay, facilitate successful and timely transition back to the community and optimize opioid monitoring/weaning post discharge. This presentation will introduce the various pharmacological management techniques employed by the specialists and present novel data with a focus on the successes and challenges of implementing the TPS over the first year.

\section{C}

\section{PSYCHOLOGICAL INTERVENTION FOR POST-SURGICAL PAIN IN THE TRANSITIONAL PAIN SERVICE \\ Aliza Weinrib \\ Toronto General Hospital, Toronto, Ontario}

Factors that to contribute to severe, acute post-surgical pain begin with incision and extend into the intra-and post-operative phases of the perioperative period. These factors which include intra-operative tissue damage, the ensuing inflammatory cascade, and possible nerve damage, require early, intensive treatment with multimodal analgesia. While these powerful medicines control pain after surgery, they do not eliminate pain entirely. The pain that remains is a complex mind-body phenomenon, which is influenced by a patient's mood, attention, expectations, past experience, and other psychological factors. In the Transitional Pain Service, psychological interventions have been designed to target these psychological and emotional factors in order to reduce pain intensity, mood disturbance, pain-related disability, and to help patient's cope with the challenges that arise when they return home. The goal of psychological treatment is to act in combination with the other aspects of our multidisciplinary approach to impact the patient's pain trajectory and prevent persistent, long-term pain and disability after surgery. To that end, we have adapted mindfulness- and acceptance-based pain management strategies to the post-surgical context to treat both acute and persistent pain. These strategies are used in combination with behavioral interventions drawn from Acceptance and Commitment Therapy that highlight personal values and goals during recovery. Case examples will be utilized to illustrate this novel psychological approach to managing acute and persistent pain after surgery.

\section{Session 206: 1:30 - 3:00pm}

\section{2}

\section{CULTURAL VARIABILITY IN PAIN: IDENTIFYING EFFECTIVE} PAIN ASSESSMENT MANAGEMENT STRATEGIES

Chair: Rebecca Pillai Riddell

York University, Toronto, Ontario

Speakers: G Allen Finley, Margot Latimer, Monica O'Neill

Workshop Objective:

This multidisciplinary presentation (anesthesiology, nursing, psychology) sets out to present new research investigating cultural differences in pain expression and the factors that influence this variability.

Learning Objectives:

1. To clarify the challenges involved with understanding individual differences and cultural stereotypes in clinical practice.

2. To inform participants about the cross-cultural factors in pain assessment and management through new research in a Canadian Aboriginal context.

3. To elucidate a novel research framework for understanding cultural variability in acute pain through an examination of caregiver soothing behaviours.

\section{A}

CAPACITY BUILDING IN HEALTHCARE: CROSS-CULTURAL PERSPECTIVES OF PAIN ASSESSMENT AND MANAGEMENT

\section{G Allen Finley}

Dalhousie University, IWK Health Centre, Halifax, Nova Scotia

Pain behaviours are strongly influenced by cultural expectations and learning in early childhood. However, there is little or no evidence that pain experience or perception varies between races or cultures (Finley et al.,
2009). Research in several countries with very different cultures suggest that children experience frequent severe pain from disease and medical procedures, and that these cause similar suffering for their parents and caregivers (Forgeron et al., 2006). Experience working with local experts to improve pain management in hospitals in Northeastern Thailand, Jordan, and Brazil has informed perspectives on pain assessment and management. We will identify difficulties in assessing children's pain and the obstacles induced by the constraints of a busy tertiary care environment in lower resource environments. The presentation will identify successful approaches to implementing effective pain assessment and management strategies in developing nations, and the global and Canadian implications of this work.

\section{B}

\section{CULTURE CLASH: ABORIGINAL PEOPLE'S EXPERIENCES SEEKING PAIN CARE AND HEALTHCARE PROVIDERS' PERSPECTIVES}

\section{Margot Latimer}

Dalhousie University, IWK Health Centre, Halifax, Nova Scotia

Aboriginal people experience high rates of pain conditions (Latimer et al., 2014), yet there appear to be many factors that contribute to their ability to actually receive effective care. Pertinent factors include the manner in which Aboriginal people express pain and healthcare providers' ability to assess it based on their culturally safe knowledge. Lingering effects of colonization efforts, language, and cultural beliefs inform preconceived perceptions for both the healthcare provider and the Aboriginal person seeking pain care (Latimer et al., 2014). There is recent evidence that Aboriginal people seek their chronic pain care in the Emergency Department (Browne et al., 2011), where negative stereotypes can be problematic. Both patients and health providers develop a set of beliefs and assumptions, and this may influence the quality of pain care delivered in any setting. Aboriginal people have reported feeling distrust and discrimination in seeking health care and there is evidence that health care providers' assessment of the pain of people from cultures different than their own is lower (Latimer et al., 2014). Dr. Latimer and Ms. Paul will review research examining Aboriginal people's experiences seeking health care, what is known about healthcare providers' experiences and beliefs regarding delivering pain care to this population and some of the educational facilitators and barriers that may impact safe pain care.

\section{C}

\section{UNDERSTANDING THE RELATIONSHIP BETWEEN CULTURE AND INFANT PAIN BEHAVIOUR: ARE CAREGIVER BEHAVIOURS THE MECHANISM?}

\section{Monica $\mathbf{O}^{\prime}$ Neill}

\section{York University, Toronto, Ontario}

Infants are vulnerable to improper pain management due to their inability to verbally communicate pain (Pillai Riddell \& Racine, 2009). The complexity of assessing pain in infancy is exacerbated when compounded by cultural differences in pain expression, assessment, and management. Although past studies have examined individual relationships between culture and caregiver pain management behaviours (Vinall et al., 2011), or culture and infant pain expression (Rosmus et al., 2000), the interplay of these variables has not been examined in one unified model. After providing a review of research in the field to date, this talk will present data from a new study examining if caregiver soothing behaviours are the mechanism by which culture predicts infant acute pain (O'Neill, Pillai Riddell et al., submitted). An innovative approach to defining culture was used that incorporated an objectively derived individualism-collectivism rating of the caregiver's self-identified heritage culture and also the caregiver's actual strength of cultural identification. Infant and caregiver behavior from the 12-month wave of the OUCH Cohort (Pillai Riddell et al., 2011; $n=393$ ) was analyzed. Results demonstrated that caregivers with stronger identification with individualistic cultures tended to show greater emotional availability, which in turn predicted lower infant pain expression at 1 and 2 minutes post-needle. These caregivers also showed more proximal soothing behaviours, which in turn predicted higher infant pain at 1 minute only. This presentation will discuss the implications of the novel finding that caregiver culture impacts infant pain indirectly and present a novel theoretical framework to stimulate audience-led discussion. 
Session 207: 1:30 - 3:00pm

\section{3}

\section{UNRAVELING THE THREAD OF NATURE VIA NURTURE - THE IMPACT OF SOCIAL RELATIONSHIPS AND CONTEXT ON GENE EXPRESSION AND PAIN RESPONSE}

Chair: Ian Weaver

Dalhousie University, Halifax, Nova Scotia

Speakers: Ian Weaver, Marsha Campbell-Yeo, Loren J Martin

Workshop Objective:

We propose a symposium to discuss the important influence of social relationships and environmental context on pain response and gene expression. In addition to our findings, we will discuss issues related to the feasibility and conduct of animal as well as human studies examining these questions. Our goal is to engage clinicians with pain neuroscientists to address how basic and clinical scientists can best address these complex questions.

Learning Objectives:

1. Upon completion of this session, attendees will be able to discuss the effect of early mother-offspring interactions controlled using psychological and physical stress modelling on later genome integrity and gene expression programs that underlie normal cognitive and emotional development.

2. Upon completion of this session, attendees will be able to understand the potential role of maternal contact provided during early pain on epigenetic marks on specific neural networks mediating pain and stress responses.

3. Upon completion of this session, attendees will be able to discuss the influence of social and environmental stressors on stress and pain response.

\section{A}

\section{EFFECTS OF EARLY ADVERSITY ON MATERNAL CARE AND EPIGENETIC MECHANISMS MEDIATING LONG-TERM DEVELOPMENT: A NEW ROLE FOR THE CHROMATIN- REMODELLING PROTEIN ATRX}

\section{Ian Weaver}

\section{Dalhousie University, Halifax, Nova Scotia}

Mounting evidence indicates that the maintenance of chromatin architecture is essential for normal human development. The ATRX gene, a chromatin-remodeling protein expressed in neurons early in development, is important for genomic integrity to buffer the constant attack on genetic material from numerous endogenous and exogenous agents (e.g. stress). Employing a new model of gestational psychological stress, with well-established models of physical stress, we have shown that maternal behaviour mediates the effects of gestational psychological stress on neural ATRX gene expression in the offspring, which is associated with alterations in DNA methylation, DNA damage signaling and stable individual differences in learning, memory and anxiety-related and social behaviour. These results and those from cross-fostering studies support the possible involvement of ATRX in the sensitization of neurons to stress hormones and the programming of somatic behaviour in response to maternal care. Since disruption of ATRX contributes to developmental silencing of imprinted genes, we hypothesize that the effects of mother-offspring interactions during the first week of postnatal life on ATRX expression and function influences both genome integrity and gene expression programs that underlie normal cognitive and emotional development. Understanding mechanisms involved in the impact of neonatal nurturing addresses perhaps the most challenging question in development: How are experiences occurring in early life rendered permanent? In the case of genome stability and sustained changes in gene expression in brain cells, we can begin to understand the neurobiological basis for individual differences in emotion, personality and social cognition that profoundly contribute to pain perception and behavior.

\section{B}

\section{EPIGENETIC IMPACT OF MATERNAL SKIN-TO-SKIN CARE DURING EARLY PAIN EXPOSURE IN HEALTHY AND PRETERM INFANTS \\ Marsha Campbell-Yeo \\ Dalhousie University, Halifax, Nova Scotia}

The quality of positive attachment in parent (typically mother)-infant bonding has profound influences on long-term emotional and psychological health. Related to this application, mothers have been shown to play a role in how the offspring react to pain and stress. For example, maternal contact has analgesic effects on newborns1 Animal models of maternal care also show mothers buffer the pain experience, that increased maternal licking and grooming (LG) reduces stress responsively, while tactile stimulation via suckling and skin contact with the dam reduces pain responsiveness. In vivo and ex vivo evidence suggests that neonatal touch can cause persistent changes by epigenetic reprogramming of gene expression patterns and permanently alter subsequent reactivity to stressors. To date, these studies have primarily focused on rodent model systems. The question then becomes whether human skin-to-skin contact might directly alter the development and function of neuroendocrine systems that regulate pain and stress responses in humans as well as the gene expression. Preliminary findings from two randomized trials conducted to determine the potential impact of early repeated procedural pain-related stress in relation to epigenetic programming of pain/stress-related genes in the blood of healthy term infants (considered normal baseline) and preterm infants (< 37 weeks gestation at birth) and the role maternal skin contact compared to $24 \%$ sucrose as interventions to relieve pain/stress will be discussed2. Understanding the maternally-induced epigenetic marks on specific neural networks mediating pain and stress responses is key to understand the long term outcomes and the protective function of maternal care.

\section{C}

\section{THE MODULATION OF PAIN BY SOCIAL AND ENVIRONMENTAL STRESS}

\section{Loren J Martin}

\section{University of Toronto, Toronto, Ontario}

The modulation of pain by stress and emotion is now widely recognized as important for adaptation. Stress, depending on its nature, duration and intensity can exert profoundly complex changes on pain states typified by either a reduction (analgesia) or exacerbation (hyperalgesia). In humans, psychological factors such as stress, anxiety and expectation play an important role in shaping pain perception, and in the clinic, when pain is anticipated, patients often report heightened pain sensations. Thus, behaviours associated with pain may not be intrinsic to the stimulus of pain, but may be a response to external stressors such as social and/or environmental reinforcers. This talk will focus on the use of novel animal models for the study of stress-induced hyperalgesia and recent efforts in our lab to translate these findings to people. In particular we find that familiarity between conspecifics is necessary for a form of empathy and that increased stress hormones prevent the expression of empathy between strangers. Additionally, we also have shown that mice and people become sensitized to their environment when they have had an aversive pain experience within that environment. This sensitization persists for at least 24 hours, is only present in males of both species and is dependent on the stress response. These models provide a new means for studying the relationship between stress and pain by examining the influence of social and environmental stressors in both mice and people. 


\section{4}

EXPLORING NOVEL MECHANISMS OF MUSCULOSKELETAL PAIN

Chair: Jason J McDougall

Dalhousie University, Halifax, Nova Scotia

Speakers: Jason J McDougall, Brian Cairns, Laura Stone

Workshop Objective:

The aim of this symposium is to highlight emerging neurophysiological mechanisms responsible for the generation and maintenance of musculoskeletal pain using preclinical animal models of disease.

Learning Objectives:

1. Hear about the emerging evidence indicating that osteoarthritis pain has a neuropathic component.

2. Learn about the cross communication between glial cells and sensory nerves in the maintenance of chronic muscle pain.

3. Have an appreciation of the relationship between intervertebral disc degeneration and the development of low back pain.

\section{A}

\section{NEUROPHYSIOLOGICAL EVIDENCE OF A NEUROPATHIC COMPONENT OF OSTEOARTHRITIS PAIN}

\section{Jason J McDougall}

\section{Dalhousie University, Halifax, Nova Scotia}

It has recently been hypothesised that there is a neuropathic component of OA which would explain why a number of classical analgesics fail to alleviate $\mathrm{OA}$ pain in some patients. This presentation will highlight the prevailing clinical and preclinical evidence which show that OA joints exhibit signs of peripheral neuropathy and that OA patients describe neuropathic pain-like symptoms. The effectiveness of neuropathic pain drugs in OA will be discussed. Recent findings showing some of the potential mechanisms involved in OA-related neuropathy and the development of neuropathic pain in preclinical models of disease will also be presented.

\section{B \\ THE ROLE OF SATELLITE GLIAL CELLS IN THE CHRONICITY OF MUSCLE PAIN \\ Brian Cairns \\ University of British Columbia, Vancouver, British Columbia; Aalborg University, Aalborg, Denmark \\ Sensory ganglia contain ganglion neurons and satellite glial cells (SGCs). The ganglion neuron is surrounded by SGCs, with a gap of about $20 \mathrm{~nm}$ between the neuronal and SGC cell membranes. This session will discuss a novel mechanism that may underlie chronic muscle pain: SGC-neuronal cross-talk in the sensory ganglion.}

\section{C}

\section{THE RELATIONSHIP BETWEEN PROGRESSIVE DISC DEGENERATION, PATHOLOGICAL DISC INNERVATION AND BEHAVIOURAL CHANGES IN A RODENT MODEL OF LOW BACK PAIN \\ Laura Stone}

\section{McGill University, Montreal, Quebec}

Dr Stone will present recent pre-clinical findings on the role of the intervertebral discs in low back pain. The relationship between progressive disc degeneration, pathological disc innervation and the emergence of behavioural signs of low back and radicular leg pain will be discussed. The therapeutic benefits of increased physical activity on these parameters will be presented and potential mechanisms underlying the beneficial effects of exercise will be proposed.

\section{5}

PAINLESS SOCIAL MEDIA FOR SCIENTISTS AND CLINICIANS: LESSONS LEARNED

Chair: Michael McGillion

McMaster University, Hamilton, Ontario

Speakers: Lynn Cooper, Rob McEwan, Michael McGillion

Workshop Objective:

Social media is becoming a core strategy to communicate health information and disseminate latest research findings. Yet, there remains ambiguity about social media in terms of how to use it effectively, despite continued pressure for researchers and clinicians to have a strong social media presence in the public domain. This symposium will be of interest to researchers and clinicians who wish to understand more about effective social media engagement.

Learning Objectives:

1. Understand how social media platforms can be used to disseminate health research to patients and healthcare professionals.

2. Understand how social media platforms can be used to engage and converse with patients, healthcare professionals, and members of the public.

3. Understand how social media can be used to drive end-user traffic to research and health information websites.

\section{A}

SOCIAL MEDIA FOR PROMOTING KNOWLEDGE EXCHANGE AMONG INTERESTED MEMBERS OF THE PUBLIC

\section{Lynn Cooper}

Canadian Pain Coalition

The use of social media for public/patient outreach and engagement concerning pain and pain management issues will be presented from the perspective of a person living with pain, as well as that of a national pain organization. Topics discussed will include the current media climate, opportunities, caveats, and lessons learned regarding the use of Facebook, Twitter and other social media platforms. Key emphasis will be placed on techniques for improving access to medically-vetted education and resources, promoting knowledge exchange among interested members of the public, increasing public awareness of pain issues, advocacy for improved pain management, and instigation of positive change in societal attitudes and health systems. Lessons learned will be presented keeping central the ultimate goal of improved quality of life for people of all ages living in pain, their families and caregivers. The experiences of the Canadian Pain Coalition and its member organizations will be shared.

\section{B}

HEALTH-FOCUSED SOCIAL MEDIA, "DOS" AND "DON'TS" FOR CONTENT CREATION AND USER ENGAGEMENT

Rob McEwan

Argyle Communications

Research and medical information has traditionally been delivered exclusively from healthcare professionals or through traditional media channels (i.e., television). New social media platforms such as Facebook and Twitter are seeking to change this as complex medical information in the form of articles, website links, videos, as well as online conversations are now taking place and are shared across vast online networks of followers. Creating a social media account is easy but maintaining an active and engaged network can be challenging. This presentation will discuss five "Dos" and five "Don'ts" for researchers, healthcare professionals and organizations with, or looking to create, social media platforms. These tips will help with: creation of strong content, effective distribution of content, and consistent end-user engagement. Using the case example a new health-focused social media platform, @CardiacPainRC, we will discuss these tried and tested methods and lend advice about how to strengthen online knowledge platforms and how to drive end-user traffic and engagement, effectively. 
25C

PERFORMANCE METRICS OF A WEB-BASED CARDIAC PAIN KNOWLEDGE DISSEMINATION PLATFORM FUELED BY SOCIAL MEDIA

Michael McGillion

McMaster University, Hamilton, Ontario

As survival rates from coronary artery disease (CAD) increase, the prevalence of persistent cardiac pain-related conditions is on the rise. As a prototype for large-scale, web-based knowledge dissemination, Cardiacpain. net was designed to raise awareness of the latest research on persistent forms of cardiac pain. The multimedia design features, social media-based dissemination strategies, and patient engagement techniques employed by the Cardiacpain.net team will be presented, followed by discussion of standard and customized 12-month dissemination metrics. Linear and Poisson regression analyses will also be presented to examine the influence of social media type, design features, region of origin, and operating systems on customized dissemination metrics.

\section{Session 210: 4:00 - 5:30pm}

\section{6}

\section{INNOVATIVE CANCER PAIN METHODS}

Chair: Jordi Perez

Alan Edwards Pain Management Unit, McGill University Health

Centre, Montreal, Quebec

Speakers: Paul Hrkal, Catherine Smyth, Jordi Perez

Workshop Objective:

During this symposium three pioneering different strategies to achieve adequate pain control in cancer patients will be presented.

Learning Objectives:

1. Measure the effectiveness of specialized palliative care approaches. Development and testing potential models for the provision of palliative care. Validating existing measures of palliative care effectiveness and creating new ones. A particular focus on the early involvement of palliative care services in the outpatient setting.

2. Complex cancer pain management using therapeutic options above the WHO Analgesic Ladder (e.g. epidural and spinal catheters for refractory cancer pain) Indications and contraindications of spinal drug delivery in cancer pain cases Ambulatory management of spinal drug delivery systems in advanced cancer pain patients.

3. Interdisciplinary approaches to cancer pain management. Pros and cons Description of an interdisciplinary model of cancer pain clinic Outcomes in terms of pain relief and other symptoms improvement.

\section{A}

\section{AN INTRODUCTION TO INTEGRATIVE AND NATURAL COMPLEMENTARY CANCER CARE WITH A SPECIAL FOCUS ON CANCER RELATED PAIN}

\section{Paul Hrkal}

Pain and Wellness Centre, Vaughan, Ontario

In recent years, more patients are interested in natural and alternative cancer therapies to complement their conventional cancer care. Dr Paul Hrkal will introduce the emerging evidence supporting the benefits of using diet, exercise and certain natural substances as part on an integrative cancer treatment plan. Dr. Hrkal will discuss natural approaches such as intravenous vitamin $\mathrm{C}$ infusions and other natural therapies as coadjuvant treatments during chemotherapy and radiation therapy. The most common questions and concerns about the safety and synergy with conventional therapies will be discussed. A special focus of this presentation will be on natural strategies to prevent and address cancer related pain, namely chemotherapy induced neuropathy. The role of the mitochondria in the pathophysiology of neuropathy will be explored as well as innovative approaches to address mitochondrial dysfunction in chronic pain.

\section{B}

INTRATHECAL DRUG DELIVERY IN CANCER PAIN AT END-OF-LIFE: EXPERIENCE OF THE OTTAWA HOSPITAL COMPLEX CANCER PAIN PROGRAM

\section{Catherine Smyth}

The Ottawa Hospital, University of Ottawa, Ottawa, Ontario

Pain is still a common and feared symptom of cancer with prevalence rates of $64 \%$ in patients with advanced disease and more than one third of these rating their pain as moderate or severe. Unfortunately conventional medical management informed by guidelines and expert opinion might still leave $10-15 \%$ of patients with inadequate pain control at end of life. The Ottawa Hospital Cancer Centre has approached refractory cancer pain management with an interdisciplinary complex cancer pain program trialing options above the World Health Organization (WHO) ladder, such as intrathecal analgesia. This speaker will focus on the development and implementation of the intrathecal program in Ottawa, including specialized care in the community. The speaker will also review the benefits and harms of intrathecal analgesia in 46 patients that received intrathecal catheters for cancer pain management from 2011-2013 at The Ottawa Hospital. Informed by this Quality Improvement initiative, there will be discussion of the necessary supports required to manage patients pre- and post-insertion of IT catheter.

\section{C}

\section{THE INTERDISCIPLINARY APPROACH TO ASSESS AND MANAGE CANCER PAIN \\ Jordi Perez}

Alan Edwards Pain Management Unit, McGill University Health Centre, Montreal, Quebec

Cancer-related symptom management is best achieved by interdisciplinary teams. McGill University Health Centre (MUHC) started an interdisciplinary Cancer Pain Clinic (CPC) in 2011. This initiative includes coordinate treatment provided simultaneously by three physicians (palliative care, anaesthesiologist and radiation oncologist) and a nurse specialist in oncology and palliative care. The treatment is complemented with rapid access to physiotherapy, occupational therapy and psychosocial oncology when deemed necessary.

The speaker will present a retrospective analysis of the initial clinical response of cancer pain patients seen at least three times at MUHC's CPC. In brief, following interdisciplinary cancer pain care, results show the following: 1) Significant and meaningful pain relief; 2) Improved pain-related limitations; 3) Significant relief of other cancer-related symptoms; 4) A trend to prescribe and consume less opioid medication

A detailed description of treatments offered at MUHC's CPC will be provided with special attention to how to combine pharmacologic and nonpharmacologic approaches. A discussion of pros and cons of the MUHC's $\mathrm{CPC}$ interdisciplinary cancer pain approach will be provided after reviewing the current evidence of this treatment.

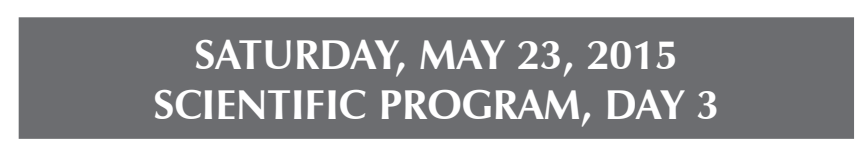

\section{Plenary Session: 9:00 - 9:45am}

\section{8}

\section{PAIN CATASTROPHIZING: TIME FOR V2.0?}

Speaker: Jennifer A Haythornthwaite

The Center for Mind-Body Research, Johns Hopkins University

School of Medicine, Baltimore, Maryland, USA

For more than two decades pain catastrophizing has been shown to have generally negative effects on various important clinical outcomes in a wide array of models ranging from laboratory pain processing in healthy volunteers to long-term pain in patients following surgery. The neurobiology of pain catastrophizing includes both peripheral and central processes that in part 
account for the increased pain sensitivity frequently observed in both healthy volunteers and clinical samples, while laboratory data underscore the role that situational pain catastrophizing plays in increasing pain sensitivity. Clinical data suggest associations with outcomes that include pain-related impairment, analgesic use, and persistence of pain over months and years. This talk will present some of the most recent research on pain catastrophizing with a discussion of what is missing, highlighting factors to be addressed in future research. Some key measurement issues include the utility of dispositional vs. situational measures and the value of manipulating the referent when measuring pain catastrophizing. The magnitude of the effect of catastrophizing on multiple clinical outcomes is generally modest and future work needs to continue to identify factors that mitigate the negative effects of pain catastrophizing. Psychological interventions can effectively reduce catastrophizing and we need to test which clinical approaches maximize clinical outcomes in targeted high risk subgroups and investigate the potential benefits of risk reduction interventions within the spectrum of pain management approaches that include pharmacological and surgical treatments. The next generation of pain catastrophizing studies provides exciting opportunities to extend our knowledge of how, when, and in whom we can improve clinical outcomes in groups at risk for persistent pain and pain-related disability.

Learning Objectives:

1. Identify two pathophysiological mechanisms linking pain catastrophizing to clinical outcomes

2. Compare the strengths of different measures of pain catastrophizing

3. Discuss a surgical model in which reducing pain catastrophizing pre- or postoperatively might improve clinical outcomes

\section{Distinguished Career Award Lecture: 9:30am - 10:00am}

\section{9}

\section{WHAT WE HAVE LEARNED AND WHAT WE STILL DON'T KNOW ABOUT VISCERAL PAIN}

\section{Speaker: Fernando Cervero}

Anaesthesia Research Unit, McGill University, Montreal, Quebec

Visceral pain is the most common form of pain produced by disease and one of the most frequent reasons for patients to seek medical care. For a long time, visceral pain was simply regarded as a variety of somatic pain, produced by similar mechanism and treated like any other form of pain. Yet in the past decades we have made considerable progress in our understanding of visceral pain mechanisms, and have realized that it is mediated by different and sometimes unique mechanisms both in the periphery and at the CNS level. We have unraveled the properties of visceral nociceptors and other sensory receptors involved in pain signaling, the pathways and neurons that transmit these signals throughout the brain and the regions of the brain involved in visceral pain perception. Yet, much remains to be discovered, particularly the pathogenesis of those forms of internal pain without apparent peripheral damage or the relationship between stress, the emotional state of the individual and the perception of visceral pain.

Session 301: 11:00am - 12:30pm

\section{0}

\section{ONE YEAR LATER: AN UPDATE ON THE ECHO ONTARIO CHRONIC PAIN PROJECT}

Chair: Ruth Dubin

Chronic Pain Committee

Speakers: Joanna G Katzman, Mandy McGlynn, Andrea Furlan

Workshop Objective:

The ECHO model is being adopted throughout North America and worldwide for many complex chronic medical conditions. ECHO Ontario is the first replication in Canada, with a mandate restricted to the province of Ontario This symposium aims to share the model with Canadian Pain Society attendees who may wish to adapt the model to their jurisdiction.
Learning Objectives:

1. To recognize the ECHO principles that lead to wider dissemination of specialist knowledge to primary care.

2. To understand how case-based, 'learning-by-doing' and creation of virtual communities of practice demonopolizes knowledge and improves patient outcomes.

3. To evaluate potential applications of an ECHO model in other regions for many different complex chronic conditions.

\section{A \\ EXPANDING HEALTHCARE ACCESS THROUGH EDUCATION: PROJECT ECHO}

\section{Joanna G Katzman}

University of New Mexico School of Medicine, UNM Pain Center, Project ECHO Pain, Albuquerque, New Mexico

Project ECHO (Extension for Community Healthcare Outcomes) is an evidence based model that provides high quality medical education for common and complex diseases through telementoring and co-management of patients with primary care clinicians. In a one to many knowledge network the ECHO model helps to bridge the gap between primary care clinicians and specialists by enhancing the knowledge, skills, confidence and practice of primary care clinicians in their local communities. As a result patients in rural and urban underserved areas may receive best practice care without long waits or having to travel long distances. Telementoring occurs through ongoing consultations via case-presentations during the videoconferences. The ECHO model was approved in 2014 by the Ministry of Health in Ontario for Chronic Pain and Opioid Stewardship. ECHO Pain provides pain education for primary care clinicians. The program improves pain assessment skills, opioid prescribing practices, confidence, and attitudes in a community of practice while providing no-cost continuing medical education. A new replication tool was developed by the UNM Project ECHO Pain team in collaboration with the US Army Medical Command to ensure a high fidelity replication of the model. The "Hub Readiness Replication Model" was created to: 1-Ensure fidelity of the Four-Point ECHO Model, 2-Create consistency in the replication process, and 3- Be adaptable for use by all ECHO disease-specific clinical and nonclinical ECHO replication programs. This replication tool has the potential to improve the fidelity of ECHO replication efforts around the world.

\section{B}

ADDRESSING THE BARRIERS TO CHRONIC PAIN MANAGEMENT: BUILDING CAPACITY AND CREATING VIRTUAL INTERPROFESSIONAL TEAMS THROUGH TELEMEDICINE

\section{Mandy McGlynn}

Toronto Rehab Hospital, University Health Network, Toronto, Ontario

A number of barriers stand in the way of effective chronic pain management. Common obstacles faced by clinicians include access to the appropriate resources and gaps in knowledge. Further, chronic pain is ultimately best managed by an interprofessional team, yet many facilities lack this opportunity for collaboration. Ontario's Extension for Community Healthcare Outcomes $(\mathrm{ECHO})$ Project is a model for providing education, expertise and consultation via telemedicine/videoconferencing. This presentation will start by introducing ECHO's innovative hub-and-spoke architecture. It will then walk you through the flow and structure of a typical weekly session, describing the curriculum, identifying the team players at both the hub and spoke and providing a sense of the kinds of complex chronic pain cases reviewed during these sessions. Although many health care practices lack access to teams for collaboration, more family practices are moving to interprofessional service delivery models, a development highlighted by this ECHO project. The presentation will explore the interprofessional representation that exists not only at the hub but also at the spokes, and demonstrate how the teaching and learning flows in both directions. The collaboration is truly interprofessional: there is no hierarchy and no one profession dominates the discussion. ECHO is a fabulous initiative that addresses challenging barriers to effective pain management and acts as a force multiplier to expand the community of professionals available to collaborate on chronic pain management. 
30C

REPLICATION OF A SUCCESSFUL MODEL: THE EXPERIENCE AND CHALLENGES OF THE ECHO ONTARIO

\section{Andrea Furlan}

University of Toronto, Faculty of Medicine, Toronto, Ontario

ECHO Ontario Chronic Pain and Opioid Stewardship launched in June 2014 to address the specific needs of our provincial population. With the vision of better chronic pain management for primary care providers and in close collaboration with the University of New Mexico ECHO Pain, ECHO Ontario secured funding to develop core infrastructure, personnel, and outcome evaluations. This presentation will discuss the adaptation of the $\mathrm{ECHO}$ model to Ontario, outline research initiatives underway to measure the impact of ECHO Ontario, and report preliminary results to date. ECHO Ontario measures impact at three different levels: primary care provider, patient, and system. We aim to increase primary care provider capacity by creating sustainable networks of interprofessional care, increasing knowledge and confidence, informing attitudes regarding opioid and addictions management, and reducing rural isolation in underserved communities. On the patient level, we aim to measure changes in care, pharmacological and nonpharmacological management, and functional improvements. On the systems level, we are collaborating with the Ministry of Health to assess patient economic impact. Adaptability and fidelity to the ECHO model in other chronic medical conditions will also be discussed.

\section{Session 302: 11:00am - 12:30pm}

31

NETWORK META-ANALYSIS: A PAIN(LESS) PRIMER

Chair: Jason Busse

McMaster University, Hamilton, Ontario

Speakers: Sohail Mulla, Sun Makosso Kallyth, Jason Busse

Workshop Objective:

Network meta-analysis is an increasingly popular approach to evidence synthesis that allows researchers to estimate the relative benefits and harms of therapies that have not been tested directly against each other in clinical trials. This symposium aims to introduce the audience to network meta-analysis, specifically in context of pain prevention and management research.

Learning Objectives:

1. To present an overview of the evolution of evidence synthesis methods in clinical research, with an emphasis on optimizing evidence-based management of patients with pain.

2. To introduce the audience to network meta-analyses, and alert them to methodological challenges for pain research.

3. To illustrate a network meta-analysis of treatment for fibromyalgia.

\section{A}

\section{THE EVOLUTION OF EVIDENCE SYNTHESIS METHODS IN CLINICAL RESEARCH}

\section{Sohail Mulla}

\section{McMaster University, Hamilton, Ontario}

A key facet of evidence-based medicine is the use of systematic summaries of the current best evidence to inform the care of individual patients. Systematic reviews are often accompanied by meta-analyses, which provide pooled estimates of effects for healthcare interventions. Meta-analyses are traditionally restricted to treatments that have been directly compared in clinical trials (e.g. opioid vs. placebo). For many pain conditions, however, there are several interventions that have not been compared against one another. This paucity of direct evidence makes it difficult to estimate treatments' relative benefits and harms, thereby decreasing the utility of the published evidence for patients, clinicians and policy-makers. In the absence of direct evidence comparing two treatments - say A and B - it is possible to calculate the indirect relative effects of $A$ versus $B$ by way of a common comparator - say $\mathrm{C}$ - against which both $\mathrm{A}$ and $\mathrm{B}$ have each been tested. In the last decade, researchers have developed innovative synthesis methods that allow combining evidence from direct and indirect comparisons. The resulting approach is termed network meta-analysis. Given their advantages over traditional meta-analyses, researchers are increasingly using network meta-analyses to synthesize evidence for treatment of painrelated conditions.

\section{B}

HOW TO CONDUCT, REPORT, AND USE NETWORK METAANALYSES FOR PAIN RESEARCH

Sun Makosso Kallyth

McMaster University, Hamilton, Ontario

Network meta-analysis is an increasingly popular statistical method that facilitates estimation of relative benefits and harms of treatments that have not been tested directly against each other in clinical studies. This presentation will provide a conceptual overview of network meta-analyses, including key assumptions related to their validity. It will showcase state-of-the-art methods to depict the state of the evidence (addressing a research question) in a graphical format, and to rank treatments according to their relative merits. This presentation will draw upon the pain literature to explain important topics, and discuss challenges and future research.

\section{C}

MANAGEMENT OF FIBROMYALGIA: A NETWORK METAANALYSIS OF RANDOMIZED CONTROLLED TRIALS

\section{Jason Busse}

\section{McMaster University, Hamilton, Ontario}

Fibromyalgia is a syndrome characterized by chronic widespread pain and excessive tenderness at 11 of 18 specific muscle-tendon sites, for which no clear cause can be found. Approximately 3.3\% of the general population in Canada suffer from fibromyalgia, with women affected 10 times more often than men. Treatment directed towards fibromyalgia is highly variable, and no review has evaluated all interventional studies for fibromyalgia, which limits attempts to make inferences regarding the relative effectiveness and safety of treatments. Our group is conducting a network meta-analysis of all randomized controlled trials evaluating therapies for fibromyalgia. We acquired eligible studies through systematic searches of multiple electronic databases. Eligible studies randomly allocated patients presenting with fibromyalgia or a related condition to an intervention or a control. Teams of reviewers, independently and in duplicate, screened titles and abstracts of identified citations, reviewed the full texts of potentially eligible trials, and extracted information from eligible studies. We used the recommendations by the Initiative on Methods, Measurement, and Pain Assessment in Clinical Trials to assess treatment effects across multiple outcome domains. Of 15,466 potentially eligible articles, we retrieved 763 in full text, of which 342 trials published in 12 languages proved eligible. Our results are helping us identify which therapies show evidence of effectiveness, and the relative effectiveness of these treatments. Our review will be the first to evaluate all treatments for fibromyalgia, provide relative effectiveness of treatments, and prioritize patient-important outcomes with a focus on functional gains.

\section{Session 303: 11:00am - 12:30pm}

\section{2}

\section{WORRY, FEAR, AND PHOBIA: A CROSS-CONTEXTUAL APPROACH TO CHILD FEAR AND ANTICIPATORY DISTRESS TO PAINFUL MEDICAL PROCEDURES}

Chair: Nicole Racine

York University, Toronto, Ontario

Speakers: Rebecca Pillai Riddell, C Meghan McMurtry, Jill Chorney Workshop Objective:

After a brief orientation to the field of fear and anxiety in relation to pain in childhood by the chair, presenters will review the current state of research in this area and synthesize the key factors that are related to anticipatory distress, the role of caregiver variables, and key directions for evidence-based treatment and intervention. The symposium will conclude with a participatory audience question and answer period. 
Learning Objectives:

1. Update audience knowledge on the child, caregiver, and contextual factors that predict anticipatory distress to painful medical procedures in children from infancy to adolescence.

2. Present experimental evidence using diverse methodologies to explore fear and anxiety to painful medical procedures in children.

3. Stimulate discussion regarding the equivocal role parent factors have been shown to play in anxiety related to pediatric medical procedures.

\section{A}

THE ANTICIPATORY MEDICAL PROCEDURE DISTRESS MODEL: UNDERSTANDING DEVELOPMENTAL PATHWAYS TO PEDIATRIC FEAR PRIOR TO PAINFUL PROCEDURES

\section{Rebecca Pillai Riddell}

\section{York University, Toronto, Ontario}

Results from a recent large-scale systematic review on the factors predicting anticipatory distress to painful medical procedures in children (Racine, Pillai Riddell et al., submitted). Using PRISMA standards to guide the review, 7084 abstracts were reviewed and 82 studies were included. The final studies all explored factors that predict anticipatory distress in infants to adolescents and a variety of medical procedures including surgeries, routine acute painful procedures (e.g. immunization and venipuncture), and procedures for chronic illness (e.g. lumbar puncture and bone marrow aspiration). A synthesis of these articles has led to a number of new insights regarding the predisposing, precipitating, perpetuating, and present factors that predict anticipatory distress and fear to pain in children. Based on the results from the review, the Anticipatory Medical Procedure Distress (AMPD) model will be presented and discussed as a conceptual framework for understanding the development of anticipatory distress. Finally, exciting new research from the $\mathrm{OUCH}$ Cohort examining individual child and parent factors from the child's first year of life that predict the development of anticipatory distress to immunization at the preschool age will be presented and interpreted in the context of the AMPD model.

\section{B}

\section{NASTY NEEDLES? CHILDREN'S PERCEPTIONS AND EXPERIENCES OF NEEDLE PROCEDURES}

\section{Meghan McMurtry}

\section{University of Guelph, Guelph, Ontario}

Fear of needles is common across the lifespan. Needle fear can range from mild to severe. At the extreme end of the spectrum, individuals present with impairments in functioning, and/or avoidance of needle procedures or even related situations such as general medical care; this is considered to be representative of a "needle phobia". Across a number of needle procedures, fear has shown a positive correlation with pain experienced. The current talk will summarize our research program examining school-aged children (5-12 years) and adolescents' (13 years and older) perceptions and experiences of needle procedures. These studies employed a variety of methodologies including: clinical pain (venipuncture), experimental tasks (vignettes), and questionnaires. The severity of pain and fear that children report experiencing during needles compared with other situations will be reviewed. Children's perceptions of their experiences will be compared to other caregivers' proxy ratings including parents as well as clinicians working with children with a chronic illness. Descriptions of the aspects of needle procedures children report as fear-inducing will be detailed as well as their rankings of different needle procedures (e.g., venipuncture, immunization injection, dental injection) in terms of fear. Proposed relationship(s) between children's fear and experience of pain will be briefly reviewed, including consideration of whether levels of fear relate to when children desire intervention for their pain. Consideration of data indicating the importance of managing needle fear in children will ultimately lead to an overview of the evidence-based treatment for high levels of needle fear in children and adolescents.

\section{$32 \mathrm{C}$}

PREDICTORS OF CHILDREN'S PREOPERATIVE ANXIETY

Jill Chorney

Dalhousie University, Halifax, Nova Scotia

Induction of anesthesia has been identified as one of the most distressing aspects of pediatric surgery (Chorney \& Kain, 2009). Higher preoperative anxiety in children has been associated with negative outcomes, such as increased postoperative pain and analgesic use, more difficultly performing induction, greater incidence of emergence delirium, and greater time spent in the recovery room (Kain, Mayes, Caldwell-Andrews, Karas \& McClain, 2006). Consequently, identifying what factors are associated with child preoperative anxiety is important in order to better understanding who is at risk and how those risk factors might best be mitigated. This talk will review literature and present data on predictors of children's fear at induction of anesthesia. Data from a larger study that included 93 children (2-10 years) undergoing elective surgery at the IWK Health Centre, one parent, and their treating anesthesiologist will also be presented. In this study, parents completed measures of their child's temperament, past medical history, and their own anxiety. Before leaving for the operating room, the treating anesthesiologist provided a prediction of children's anxiety at induction.

Observational measures of child preoperative anxiety were also collected. Child preoperative anxiety was predicted by child age, aspects of temperament, previous medical visits, and anesthesiologist estimates; however, child preoperative anxiety was not associated with parent anxiety. Results of this study are largely consistent with much of past research but raise some questions about the relation between parent and child anxiety in this medical context. 
CPS POSTERS

WEDNESDAY MAY 20, 2015

P1

\section{USE OF NEUROPATHIC PAIN QUESTIONNAIRES IN PREDICTING THE DEVELOPMENT OF FAILED BACK SURGERY SYNDROME FOLLOWING LUMBAR DISCECTOMY FOR RADICULOPATHY}

\section{Mohammed F Shamji}

Toronto Western Hospital, Toronto, Ontario

POSTER AIM: Failed back surgery syndrome (FBSS) describes neuropathic pain that occurs when extremity symptoms in lumbar disease persist despite structurally corrective spinal surgery. It is unclear whether specific preoperative pain characteristics predict which patients are prone to this postoperative persistence of their disabling symptoms.

METHODS: This prospective study analyzed surgical patients with painful radiculopathy secondary to lumbar degenerative disease. Clinical parameters included general demographic information, preoperative and postoperative clinical examination, self-reported pain and disability scores, and neuropathic pain scoring. The neuropathic pain screening tests used in this study were the Douleur Neuropathique 4 (DN4) and Leeds Assessment of Neuropathic Symptoms and Signs (LANSS), with correlation tested for ordinal score and screen positivity. Multiple logistic regression analysis was used to define predictors of postoperative symptomatology.

RESULTS: Among 250 surgical radiculopathy patients, 12\% were classified with FBSS with only modest relief of leg pain. The condition was highly associated with abnormal preoperative screens for neuropathic pain, but not gender, smoking status, or preoperative pain severity (multiple logistic regression, $\alpha=0.05$ ). Good correlation was seen between the two screening tests used in this study for both absolute ordinal score (Spearman's $\rho=0.84, p<0.001)$ and thresholding for terming the patient as having neuropathic pain features (Spearman's $\rho=0.48, \mathrm{p}<0.001$ ).

CONCLUSIONS: While FBSS was more common among younger and female patients, it occurred with low overall frequency in this population. Higher neuropathic pain screening scores correlated strongly with likelihood of significant postoperative leg pain. Further work is required to develop more accurate prognostication tools for radiculopathy patients undergoing structural spinal surgery.

\section{P2}

PELVIC GIRDLE MYOPATHY CAUSED BY ISOTRETINOIN: CASE REPORT AND DISCUSSION OF PATHOPHYSIOLOGY Muhammad Umair Khan ${ }^{1}$, A Bruce Janati

${ }^{1}$ Dow University of Health Sciences, Karachi, Pakistan

POSTER AIM: Isotretinoin is the mainstay therapy for severe and nodulocystic acne. Myalgia and muscle stiffness have been reported in $16-51 \%$ of patients treated with isotretinoin, while elevated serum creatine kinase (CK) levels have been found in up to $41 \%$. In this paper, we report, for the first time, the occurrence of pelvic girdle myopathy in a teen who received isotretinoin therapy.

METHODS: This was clinical and laboratory based case study.

RESULTS: Histopathology and other laboratory data were consistent with an inflammatory myopathy based on a vasculitic process.

CONCLUSIONS: Our data indicate that the myopathy caused by isotretinoin is an inflammatory process based on vasculitis, resulting in ischemic muscle necrosis.

\section{P3}

\section{MATERNAL SENSITIVITY, MATERNAL SOOTHING BEHAVIOURS, AND INFANT PAIN DURING INFANT IMMUNIZATION}

Nicole Atkinson ${ }^{1}$, R Pillai Riddel, NM Racine, H Garfield, S Greenberg

${ }^{1}$ York University, Toronto, Ontario

POSTER AIM: This study examined whether infants who have caregivers assessed to have either extreme high or extreme low emotional availability differ in their pain scores at two, four, six, and twelve months, and whether caregivers who have either extreme high or extreme low emotional availability differ in their enacted soothing behaviours.

METHODS: Analyses were conducted with parent-infant dyads at four immunization appointments: two-months, four-months, six-months, and twelve-months.

RESULTS: T-tests and Cohen's $d$ indicated significant differences in infant pain scores, with increasing effects of parental sensitivity across age and across the appointment, and in enacted soothing behaviours of high and low sensitivity parents across age.

CONCLUSIONS: By using the extremes of sensitivity, and by examining effect sizes of all significant relationships, the current study confirmed the clinical significance of parental sensitivity in the pain context, suggesting that more sensitive parents help to alleviate their infants' distress.

\section{P4}

\section{CHARACTERISTICS OF CHRONIC NON-CANCER PAIN (CNCP) PATIENTS PRESCRIBED OPIOIDS POST} PUBLICATION OF CANADIAN GUIDELINE FOR SAFE AND EFFECTIVE USE OF OPIOIDS AT THE POINT OF ENTRY TO A TERTIARY CARE PAIN CLINIC

\section{Ahana Chatteriee ${ }^{1}$, Nivan Zoheiry, S Fatima Lakha,} Angela Mailis-Gagnon

${ }^{1}$ Toronto Western Hospital, Toronto, Ontario

POSTER AIM: To document changes in the opioid prescribing patterns of community physicians after the publication of the Canadian guideline for safe and effective use of opioids in 2010.

METHODS: Retrospective cross-sectional study of consecutive patients referred to the tertiary care pain clinic by community physicians (June 2013January 2014). Demographics, pain data and opioid treatment were collected using chart review. Patients were classified by diagnosis: pure biomedical condition accounting for pain (group I); combination biomedical and psychological conditions (group II) and psychological conditions in the absence of detectable pathology (group III), as well as by opioid use: non opioid users (NOU); and low opioid user (LOU), or high opioid users (HOU), with a daily morphine-equivalent dose (MED) of $200 \mathrm{mg}$ or more respectively.

RESULTS: Half of the cohort results were available for analysis $(\mathrm{N}=126)$. Half of all patients (51.6\%) were prescribed opioids, with $1 / 5$ exceeding 200 mg MED daily. In group I, $47.8 \%$ were opioid users with $12 \%$ been HOU. In groups II and III, $56 \%$ and $66.7 \%$ of patients were opioid users, with $25.8 \%$ and $33.3 \%$ respectively been HOU. Group II and III HOUs had a mean MED of $607.25 \mathrm{mg} /$ day versus $234.37 \mathrm{mg} /$ day in group I HOUs. CONCLUSIONS: Our results so far are nearly identical to those obtained from the cohort of 2006-2009 (Can Fam Physician 2011;57:e97-105), prior to the publication of the guideline. Cumulative results of the full cohort will be presented with face to face comparison of raw data collected in both pre- and post-guideline publication studies.

\section{P5}

\section{A PROPOSAL FOR COMPETENCY BASED EDUCATION IN ULTRASOUND GUIDED PAIN INTERVENTIONS}

Geoff A Bellingham ${ }^{1}$, Colin RJ Phillips

${ }^{1}$ Western University, London, Ontario

POSTER AIM: The use of ultrasound (US) guided interventional pain procedures has become increasingly popular. Transition to a competency based education model to teach this skill set requires sufficient exposure for US image acquisition and visualization of needle insertion and injection. Published recommendations for US-guided training in pain medicine do 
not provide estimates on the number of training images and procedures to perform. (1) Our goal is to propose these estimates based on existing pain medicine literature to begin the design of a competency framework for Pain Medicine Residency training.

METHODS: Our objective was to search the existing literature on the teaching of US-guided pain interventions, including peripheral and axial structures as well as musculoskeletal applications. This included articles from radiology, rheumatology, physiatry, and chronic pain literature. RESULTS: One feasibility study provided a learning curve for US-guided intra-articular injections to the sacroiliac joint. Success increased from $60 \%$ to $93.5 \%$ when the number performed increased from 30 to 60 blocks in the hands of experienced radiologists. (2)

CONCLUSIONS: There a paucity of literature that informs the number of image acquisition scans and procedures to construct a competency based educational program. Estimates for training should be obtained from evidence for teaching US-guided regional anesthesia, which depends on the quality of didactic sessions, learner variability, and quality of evaluation and feedback. (3) Block performance skill acquisition based on available regional anesthesia literature could be estimated to require 30 procedures. $(4,5)$. The determination of competence will be a dynamic process, based on a strong simulation program and curriculum (6).

\section{REFERENCES}

1. Narouze SN, et al. The American society of regional anesthesia and pain medicine, the European society of regional anesthesia etcâ $€$ ! . RAPM 2012;37(6):657-663.

2. Pekkafali MZ, et al. Sacroiliac joint injections performed with sonographic guidance. J Ultrasound Med 2003;22:553-559.

3. Nix CM, et al. A scoping review of the evidence for teaching ultrasound-guided regional anesthesia. RAPM 2013;38(6):471-480.

4. Rodrigues de Oliveira G, et al. Learning curves and mathematical models for interventional ultrasound basic skills. Anesth Analg 2008;106:568-73.

5. Reg Anesth Pain Med. 2012 May-Jun;37(3):334-9. doi: 10.1097/ AAP.0b013e $3182475 \mathrm{fba}$.Ultrasound-guided regional anesthesia: how much practice do novices require before achieving competency in ultrasound needle visualization using a cadaver model.

6. Niazi AU, et al. Ultrasound-Guided Regional Anesthesia Performance in the Early Learning Period. RAPM. 2012;37(1):51-54.

\section{P6}

DEVELOPMENT OF CARDIOVASCULAR INDICES OF ACUTE NEEDLE PAIN RESPONDING IN PRETERM AND TERM BORN INFANTS

\section{Jordana A Waxman ${ }^{1}$, A Pinhasov, P Tablon, R Pillai Riddell}

${ }^{1}$ York University, Toronto, Ontario

POSTER AIM: Few studies have examined the development of cardiac responses to needle pain, hindering our ability to understand and manage infant pain in term and preterm infants. The current systematic review aims to chart and compare the development of cardiovascular responses to acute needle pain in term born and preterm infants over the first year of life.

METHODS: A systematic search was conducted in MEDLINE, EMBASE, PsychINFO, and CINAHL. After removing duplicate articles, 6994 studies were reviewed for inclusion. Search results were limited to evidence-based methodologies, publication years (1970+), and age group (0-3 years). Fortyfive studies were first separated by gestational age at birth. These groups were then qualitatively synthesized based on age at measurement and cardiovascular measure (heart rate $[\mathrm{HR}]$ in beats per minute $[\mathrm{bpm}]$, heart rate variability (HRV; low frequency [LF], high frequency [HF], LF/HF ratio).

RESULTS: Across the first three months of life, although variable, median HR after needle pain was consistently higher in preterm compared to term born infants. In the first 4 days of life, both groups had decreased LF and HF HRV, and increased LF/HF ratio in response to needle pain. At 4-14 days, both groups had variable HRV responses to needle pain, however, the LF/HF ratio decreased.

CONCLUSIONS: Longitudinal research on the development of cardiovascular responses to needle pain is necessary to understand how term and preterm infants' autonomic nervous system responds to pain over time. Research in this area will lead to empirically-based evaluation and treatment of infant pain.

\section{P7}

\section{ADDRESSING SLEEP PROBLEMS IN INDIVIDUALS WITH CHRONIC PAIN}

\section{May Caprio ${ }^{1}$, L Cundiff, W Davis, D Buna}

${ }^{1}$ Island Health, Victoria, British Columbia

POSTER AIM: Chronic pain interferes with sleep and the lack of sleep interferes with the ability to tolerate pain thus creating a vicious cycle. The objective of this study was to evaluate the impact of a group program on sleep in patients with chronic non-malignant pain who were part of a tertiary referral program.

METHODS: Sixty-three participants over the age of 18 were provided a sleep group program that consisted of eight hours over four weeks. Participants were educated on the basic science of sleep and pain, how lifestyle affects sleep (exercise, light, caffeine, nicotine, alcohol and food), personal strategies for management of sleep in the context of chronic pain, sleep hygiene, cognitive behaviour therapy for insomnia and how medication affects sleep. Evaluation included self-report measures of sleep, pain, mood and daily functioning that was carried out at pretest, post-test and three month follow-up. Repeated measures analysis of variance was conducted to identify significant changes at three evaluation points.

RESULTS: There was a significant $(\mathrm{p}<0.05)$ decrease from pretest to posttest on three of the four measures including sleep, pain and mood. There was a trend in the same direction at pretest to post-test on the measure of daily functioning. Improvements were maintained on all measures three months later.

CONCLUSIONS: Individuals with chronic pain can benefit from group interventions that address sleep in the context of chronic pain. Pain programs need a better understanding of the interaction between sleep and chronic pain to provide comprehensive and effective interventions.

\section{P8}

\section{THE RELATIONSHIPS BETWEEN CHILD AND PARENT} PRESCHOOL IMMUNIZATION ANXIETY Hannah Gennis ${ }^{1}$, L Campbell, R Pillai Riddell, H Garfield, S Greenberg

${ }^{1}$ York University, Toronto, Ontario

POSTER AIM: Examine the interrelationships between child behavioural anxiety pre-needle, parent anxiety pre- and post-needle, and parent report of child anxiety pre- and post-needle during preschool immunizations.

METHODS: Parents and 4-5 year old children from a longitudinal cohort were videotaped during their preschool immunization $(n=155)$. Videotapes were coded for child behavioural anxiety pre-needle (FLACC, Merkel et al., 1997). Parents self-reported their own anxiety pre- and post-needle, as well as how much anxiety they thought their child was experiencing preand post-needle (all on a 10-point scale).

RESULTS: Child behavioural anxiety pre-needle was related to parent report of child anxiety post- needle $(r=0.31, p<0.01)$. Parent self-report of anxiety pre-needle was related to parent report of child anxiety pre-needle $(\mathrm{r}=0.33, \mathrm{p}<0.001)$, parent report of child anxiety post-needle $(\mathrm{r}=0.24$, $\mathrm{p}<0.01)$, and parent self-report of anxiety post needle $(\mathrm{r}=0.60, \mathrm{p}<0.001)$. Parent self-report of anxiety post-needle was related to parent report of child anxiety pre-needle $(\mathrm{r}=0.24, \mathrm{p}<0.01)$ and parent report of child anxiety post-needle $(r=0.51, p<0.001)$. Parent report of child anxiety pre-needle was related to parent report of child anxiety post-needle $(r=0.35, p<0.001)$. CONCLUSIONS: Parent report of their own anxiety pre- and post-needle was related to their reports of child's anxiety pre- and post-needle. Children's behavioural displays of anxiety pre-needle were only related to parent report of child anxiety post-needle, not to parent report of child anxiety pre-needle. 
P9

\section{CHRONIC SCROTAL PAIN SYNDROME: CLINICAL CHARACTERISTICS OF THIS COMMON BUT POORLY UNDERSTOOD CONDITION}

Hassan Mustaneer ${ }^{1}$, A Gordon, K Jarvi, D Paneduro, T Smeenk, S Lau ${ }^{1}$ Wasser Pain Centre, Mount Sinai Hospital, Toronto, Ontario

POSTER AIM: The goal of this study was to study the clinical characteristics of a large cohort of men with chronic scrotal pain syndrome(CSPS). METHODS: A retrospective chart review of 80 patients with CSPS who presented to the Multidisciplinary Orchialgia Clinic (MOC) at Mount Sinai Hospital was conducted. Patients' medical history, physical examination, and results of investigative and treatment modalities prior to presenting to our clinic were reviewed.

RESULTS: The mean age of pain onset was 38.06 years $(S D=13.44)$ and the mean pain severity was $6.34(\mathrm{SD}=2.33)$. The average reported pain duration was 4.42 years and most patients $(80 \%)$ reported that their pain began between 21 and 50 years of age. The pain was described as being in the scrotum only in $26.25 \%$, and radiated to adjacent areas, mainly the inguinal region, thighs, and abdomen in $62.5 \%$. The epididymis was tender in $76.25 \%$ of patients, more than twice the prevalence of tenderness being found in the testes $(36.25 \%)$, with most men reporting tenderness in the head of the epididymis $(\mathrm{n}=55)$. The most common identified cause was vasectomy $(22.5 \%)$, although the majority of patients did not have an identifiable etiology (52.5\%). Approximately $46 \%$ of patients reported neurological signs and symptoms.

CONCLUSIONS: Most patients diagnosed with CSPS have tenderness principally in the epididymis and nearly half of patients present with features of neuropathic pain. Commonly used investigative approaches are not useful for diagnosing the etiology of CSPS.

\section{P11}

INCREASED LEVELS OF FRACTALKINE IN THE TRIGEMINAL GANGLION SENSITIZE TEMPORALIS MUSCLE NOCICEPTORS TO MECHANICAL STIMULATION

\section{Brian E Cairns ${ }^{1}$, Xu-Dong Dong ${ }^{1}$, M O'Brien, P Gazerani}

${ }^{1}$ University of British Columbia, Vancouver, British Columbia

POSTER AIM: Fractalkine (CX3CL1) is a unique chemotactic cytokine that activates the $\mathrm{CX} 3 \mathrm{C}$ receptor-1 (CX3CR1). Fractalkine signalling contributes to activation of satellite glial cells (SGCs) in the dorsal root ganglia induced following peripheral inflammation Intra-ganglionic injection of fractalkine produces behavioral evidence of mechanical sensitization within 30 minutes. It is not known whether fractalkine also contributes to pain processing in the trigeminal ganglion (TG).

METHODS: A total of 29 male Sprague-Dawley rats were used. Fast blue dye was injected into the temporalis muscle of 5 rats 7 days prior to euthanization and perfusion with paraformaldehyde. The TG was removed, sliced with a cryostat (10- $\mu \mathrm{m}$ sections), and visualized with a confocal microscope. CX3CR1 and glutamine synthetase antibodies were used to assess CX3CR1 expression and identify SGCs, respectively. In 24 anesthetized rats, single unit electrophysiological recording of TG neurons (TGNs) that innervate the temporalis muscle was undertaken to examine the effect of intra-ganglionic microinjection of fractalkine $(0,5,50 \mathrm{ng}, 3 \mathrm{ul})$ on their mechanical threshold.

RESULTS: The mean expression of CX3CR1 by temporalis ganglion neurons and their associated SGCs was $43 \pm 4 \%$ and $36 \pm 9 \%$, respectively. Reduction of TGNs' mechanical threshold ( $\mathrm{P}<0.05$, RMANOVA, compared to control) by intra-ganglionic injection of fractalkine (50 ng) presented as early as 30 minutes after injection and lasted up to 3 hours.

CONCLUSIONS: Fractalkine may act either directly on TGNs and/or by activation of SGCs to induce a prolonged mechanical sensitization in temporalis muscle nociceptors. The role of fractalkine in craniofacial pain pathogenesis and the potential of targeting the CX3CR1 merit further study.

\section{P12}

MECHANICAL ALLODYNIA FOLLOWING DISC HERNIATION REQUIRES INTRANEURAL MACROPHAGE INFILTRATION AND CAN BE STRATEGIES LIMITING MACROPHAGE ACTIVITY

\section{Mohammed F Shamji ${ }^{1}$, YS Tu, MW Salter}

${ }^{1}$ Toronto Western Hospital, University of Toronto, Toronto, Ontario POSTER AIM: Disc-herniation radiculopathy arises from both mechanical compression and biochemical inflammation of apposed neural elements. The mechanisms giving rise to chronic pain in patients with non-compressive disc herniation remain unclear. This study demonstrates the role of inflammatory activation in generating a painful neuropathy phenotype after placement of heterotopic disc tissue onto rodent sciatic nerve.

METHODS: C57BL/6 mice underwent mid-thigh exposure of the sciatic nerve, with control animals exposed only and experimental animals having placement of littermate tail nucleus pulposus (NP) onto the nerve. Animals were evaluated throughout one week for mechanical allodynia, thermal hyperalgesia, cold allodynia, and gait stability. At sacrifice, immunohistochemistry was performed to identify perineural and intraneural macrophage and lymphocyte presence. The experiment was repeated under conditions of macrophage depletion or antagonized neurotrophin function.

RESULTS: Mice exposed to NP stimulation demonstrated mechanical allodynia, thermal hyperalgesia, and cold allodynia compared with controls. Intraneural macrophage infiltration was observed in this group, alongside autoreactive lymphocytes at the disc-nerve interface. This behavioural phenotype was completely blocked with macrophage depletion or antagonized neurotrophin function.

CONCLUSIONS: Non-compressive disc herniation generates pain behaviour in this animal disease model, alongside intraneural macrophage migration. Strategies to decrease perineural inflammation or maintain integrity of the blood-nerve-barrier may be effective in treating painful disc-herniation radiculopathy.

\section{REFERENCES}

1. Kawaguchi S, Yamashita T, Yokogushi K, et al. Spine (2001). Shamji MF, Allen KD, So S, et al. Spine (2009). Shamji MF, Setton LA, Jarvis W, et al. A+R (2010).

\section{P13}

\section{WHAT WORKS? COPING AND EMOTION REGULATION ON MENTAL HEALTH IN WOMEN WITH IC/BPS}

Laura Katz ${ }^{1}$, DA Tripp, JC Nickel, A Krsmanovic, A Muere, LK Carr, R Mayer, RM Moldwin

\section{${ }^{1}$ Queen's University, Kingston, Ontario}

POSTER AIM: Interstitial Cystitis / Bladder Pain Syndrome (IC/BPS) is a chronic pelvic pain syndrome associated with pain, urological symptoms and poor mental health. Although IC/BPS coping strategies have been identified, these variables have not been evaluated within a single model. The aim of this study was to reduce multiple coping and outcome variables into latent constructs, and to model their pathways onto mental health. METHODS: IC/BPS patients (female; $n=195$ ) from urology clinics completed questionnaires. Exploratory (EFA) and confirmatory factor analyses (CFA) were employed to determine construct measurement. Structural equation modeling (SEM) was used examine the pathways between coping and mental health.

RESULTS: The EFAs and CFAs reduced the variables into the following constructs: illness-focused coping (IFC; guarding, resting, asking for assistance), wellness-focused coping (WFC; exercising, self-talk, relaxing, seeking social support), emotion regulation (ER; task persistence, activity engagement, pain willingness, reappraisal, suppression) and mental health (satisfaction with life, optimism/pessimism, depression, negative and positive affect). SEM demonstrated good model fit, and a significant pathway between WFC $(0.24, \mathrm{p}<0.01)$ and ER $(0.84, \mathrm{p}<0.01)$ on mental health, but not IFC $(-0.10, \mathrm{p}=0.24)$.

CONCLUSIONS: Results from this study demonstrate that WFC and positive emotion regulation strategies are associated with better mental health outcomes. Psychosocial treatments should focus on wellness-focused behavioural coping strategies, pain acceptance as well as increased cognitive 
reappraisal, and decreased cognitive suppression. Future research should evaluate the mechanisms of these coping strategies within a pain management program.

\section{P14}

CHILD SEX DIFFERENCES IN CAREGIVERS' AND HEALTH PROFESSIONALS' VERBALIZATIONS DURING PRESCHOOL IMMUNIZATIONS

\section{Hannah Gennis ${ }^{1}$, N Racine, R Pillai Riddell, H Garfield, S Greenberg} ${ }^{1}$ York University, Toronto, Ontario

POSTER AIM: Examine caregivers' and health professionals' use of procedural and non-procedural verbalizations during preschool immunizations based on child sex; Examine child sex differences in distress behaviour postneedle; Assess the relationships between caregivers' and health professionals' verbalizations pre-needle and child distress behaviour post-needle.

METHODS: 202 children between 4 and 5 years of age ( girls $=95$; boys $=107)$ and their caregivers from the OUCH cohort were videotaped during preschool immunizations. Videotapes were coded using the CAMPIS- $\mathrm{R}$ for caregiver and health professionals' use of non-procedural and procedural (positive/neutral and negative) verbalizations pre-needle, and child non-verbal and verbal distress behaviour postneedle (cry, scream, physical resistance, verbal pain, verbal emotion, and verbal resistance duration). Reliability was conducted on $20 \%$ of cases and was 0.78 for verbal behaviours.

RESULTS: Child sex did not influence caregivers' use of procedural or non-procedural verbalizations. Health professionals used more positive/ neutral procedural verbalizations with girls, $\mathrm{F}(1,200)=7.26$, $\mathrm{p}<0.01$, and girls screamed longer post-needle, $F(1,200)=7.94, p<0.01)$. There were no significant relationships between caregiver or health professional behaviour pre-needle and child distress behaviour post-needle, however there was a trend for a positive relationship between parent positive/neutral procedural talk and child verbal pain $(\mathrm{p}<0.1)$.

CONCLUSIONS: There was preliminary evidence of child sex differences in child distress behaviour post-needle and in health professionals' use of positive procedural talk, with increased use for girls. Future research should examine the sequential relationships between caregiver and healthcare provider verbalization and child distress during preschool immunization.

\section{P15 \\ PAIN FOLLOWING UNILATERAL TOTAL KNEE \\ ARTHROPLASTY: A RANDOMIZED CONTROLLED TRIAL EXAMINING THE ANALGESIC EFFECTIVENESS OF A COMBINED ADDUCTOR-CANAL PERIPHERAL NERVE BLOCK WITH PERIARTICULAR INFILTRATION VS ADDUCTOR-CANAL NERVE BLOCK ALONE VS PERIARTICULAR INFILTRATION ALONE \\ Mona Sawhney ${ }^{1}$, H Medhian, R Bowry, B Kashin, M Bent, G Ip, L Jussaume, J Proudman, K Clarke \\ ${ }^{1}$ Queen's University, Kingston, Ontario \\ POSTER AIM: Total knee arthroplasty (TKA) is a painful surgery but it requires early mobilization for successful joint function. Therefore, effec- tive pain management is essential for rehabilitation. Multimodal analgesia including: spinal anesthetic, nerve blocks, periarticular infiltration, opi- oids, and co-analgesics have been shown to effectively manage post-opera- tive pain. One of the criticisms of nerve block is the potential to impair quadriceps muscle strength which limits mobility. Both adductor canal (AC) and peri-articular infiltration (PI) have been shown to manage pain without impairing motor function. However, it is unclear which technique is most effective. The purpose of this 3 arm trial was to examine the effect of both AC+PI vs AC vs PI. The primary outcome was pain on walking at post-operative day (POD)1. \\ METHODS: Following Ethics Board approval, patients undergoing uni- lateral TKA were approached to participate in this trial. Inclusion criteria included: 18 years or older, ASA I-III, able to speak and read English. Patients were excluded if they had a contraindication to regional anesthe- sia/local anesthetics, chronic pain not related to their knee, were using}

opioids for 3 months or longer, or had a peripheral neuropathy. The sample size was calculated based on the primary outcome, and with a $\hat{I} \pm 0.5$ and $15 \%$ attrition rate, a sample of 159 participants was required. Eligible and consenting participants were randomized into 1 of the 3 groups. On the day of surgery, the participant was admitted to the 'block room' where they received either AC block with $30 \mathrm{~mL}$ of $0.5 \%$ Ropivicaine or sham block. PI was performed intra-operatively with a $110 \mathrm{~mL}$ solution of Ropivacaine $300 \mathrm{mg}$, morphine $10 \mathrm{mg}$, ketorolac $30 \mathrm{mg}$, in normal saline. Those patients randomized to AC only received normal saline.

RESULTS: A total of 159 participants consented and 144 completed the trial. The mean age was 67 years, and $63 \%$ were female. On POD1 participants who received $\mathrm{AC}+\mathrm{PI}$ reported statistically lower pain on walking (3.3) as compared to those who received AC (6.2) or PI (4.9). Participants who received AC reported statistically higher pain scores at rest and knee flexion as compared to those who received AC+PI or PI. On POD2 participants who received AC+PI reported statistically less pain on walking (3.3), as compared to those who received AC (6.2) or PI (4.9). On POD2 there was no difference between the groups for pain at rest, or flexion. Participants who received AC used more IV PCA on POD 0. There was no difference between the groups regarding distance walked.

CONCLUSIONS: Participants who received both AC + PI reported statistically less pain on walking on POD1 and 2. There was no difference between the groups on distance walked, however, this was only reported 1 time per day and did not capture distance walked over a 24 hour period if the participant walked multiple times.

\section{P16}

THE PRODROMAL SYMPTOMS SCREENING SCALE [PS-SS]: PSYCHOMETRIC ASSESSMENT

Sheila O'Keefe-McCarthy ${ }^{1}$, S-L Guo

${ }^{1}$ Ross Memorial Hospital, Lindsay, Ontario

BACKGROUND: Canadians each year seek medical emergency treatment for report of myocardial ischemia-related chest pain. A considerable number of people experience unrecognized warning signs - prodromal symptoms - of an imminent myocardial ischemic event. Prodromal symptoms (PS) are specific or non-specific symptoms experienced days to months prior to a cardiac event. The Prodromal Symptoms Screening Scale is a nine item measure to evaluate PS in individuals with coronary heart disease (CHD).

AIM: To assess the psychometric properties of the PS-SS in men and women with CHD.

METHODS: As part of an observational, prospective, descriptive-correlational study, participants completed the PS-SS by individual interview upon admission to a community rural emergency visit for report of acute coronary syndrome. Data Analysis: Internal structure of the PS-SS was examined by principal component factor analysis with oblique rotation. Factors were abstracted based on two criteria: eigenvalues $\geq 1$ and factor loading $\geq 0.3$.

RESULTS: The PS-SS presented a two-factor structure, with accepted eigenvalues greater than 1 (factor I and II had eigenvalues of 2.23 and 1.41 and explained $24.7 \%$ and $15.6 \%$ of variance, respectively. Together the factor structure explained $40.4 \%$ of the variance. Kaiser-Meyer-Olkin measure $(\mathrm{KMO})$ of sampling adequacy was $0.64(\mathrm{p}<0.001)$. Factor 1 included: Prodromal aches or pain, dizziness, chest pain and other prodromal symptoms, with an acceptable factor loading range of 0.46 to 0.77 . Factor 2 included: Prodromal fatigue, sleep disturbance, headaches, shortness of breath, and anxiety, with factor loading range between 0.40 to 0.75 . Pearson's correlation confidence was $0.28(\mathrm{p}=0.002)$ between Factor 1 and Factor 2.

CONCLUSIONS: Preliminary psychometric evaluation of the PS-SS indicates it is a valid and reliable measure to use to screen PS in men and women with CHD. 
P17

INFLUENCE OF CANCER PAIN-RELATED BELIEFS ON TAWAINESE CANCER PATIENTS' PAIN-RELATED EXPERIENCES

Shu-Liu Guo ${ }^{1}$, D Howell, J Watt-Watson, M McGillion, YC Sung

${ }^{1}$ University of Toronto, Toronto, Ontario

BACKGROUND: Evidence suggests that patients' cancer pain-related beliefs may be contributing to pain management. There is insufficient understanding of this with in Taiwanese cancer outpatients. Purpose: To examine the contribution of cancer pain-related beliefs on the pain experience of Taiwanese cancer outpatients.

METHODS: In a cross-sectional, descriptive study, adult cancer outpatients completed: a) Chinese version of Illness Perception Questionnaire (IPQ-CaCP), b) Beliefs Medicines Questionnaire (BMQ), c) Medication Adherence Report Scales (MARS-5), and d) Taiwanese version of Brief Pain Inventory-Short Form (BPI-T). Data Analysis: Hierarchical linear regression was used to determine the independent contribution of these beliefs and analgesic use on patient's pain experience.

RESULTS: One hundred and fifty-one patients (lung 44\% and colorectal cancer 56\%) completed this survey. The mean age was 63.8, SD-11.2; males $49 \%$. Of the $151,>55 \%$ agreed that cancer pain was chronic and unpredictable with negative emotional representation. Participants (64\%) endorsed that treatment could improve pain but $47 \%$ believed that they had personal control to influence their pain. Although $64 \%$ agreed that analgesics were necessary for managing pain, $51 \%$ had reservation about analgesic use. Hierarchical linear regression identified that significant contributors to worst pain intensity were more serious consequence beliefs and less treatment control beliefs $(\beta=0.22,-0.22$, respectively, $R 2=0.27$, $\mathrm{F}=2.89, \mathrm{p}<0.01$ ). Significant predictors to pain interference included serious consequence beliefs, age, pain duration, physical performance, and worst pain intensity $(\beta=0.26,-0.2,-0.12,-0.28$, and 0.3 , respectively, $\mathrm{p}<0.05$. Overall, the independent variables explained $66 \%$ of the variance in pain interference $(\mathrm{R} 2=0.6)$

CONCLUSIONS: Cancer patients' pain-related beliefs are complex and a subjective experience. Cancer pain-related beliefs should be incorporated early into the assessment. Education and patient-focused interventions provided for individuals afflicted with cancer.

\section{P18 \\ CANADIAN PAIN COALITION REPORT CARD ON PAIN: SYSTEMATIC REVIEW OF MULTIDISCIPLINARY CHRONIC PAIN SERVICES IN CANADA}

Samantha R Fashler ${ }^{1}$, L Cooper, LC Burns, S Razavi, L Goldberg, E Oosenbrug, J Katz

${ }^{1}$ York University, Toronto, Ontario

POSTER AIM: We reviewed the published literature evaluating multidisciplinary chronic pain centres across Canada to determine the professional disciplines involved, availability to the public, and services offered.

METHODS: A systematic literature review was conducted using MEDLINE, PsycINFO, and CINAHL. Search criteria were applied to identify surveys of chronic pain management centres. Of the 1674 unique articles identified by the search strategy, 25 satisfied our criteria. Of these, three surveyed Canadian pain centres.

RESULTS: Two articles consisted of Canada-wide surveys. The third evaluated services available in Quebec. The most recent survey was conducted seven years ago. In total, 172 chronic pain clinics across Canada were surveyed. Across the surveys, mean clinic wait times ranged from four weeks to nine or more months. The most commonly treated pain conditions included low back pain, neuropathic pain, headache pain, abdominal pain, complex regional pain syndrome, neck pain, and fibromyalgia. A wide variety of health care professionals worked in pain clinics; most prominently general practitioners, anesthesiologists, and psychologists. Most clinics used a variety of interventional, physical, and psychological treatments. In one survey, it was determined that there was one pain clinic per 258,000 Canadians.

CONCLUSIONS: We identified only three surveys of chronic pain centres in Canada. Since approximately 20\% of Canadians live with chronic pain, the data indicate that there is only one pain clinic for every 51,600 sufferers.
Moreover, since the most recent survey was done more than 7 years ago, there is a need for updated information about professional composition, availability, and locations of chronic pain clinics across Canada.

\section{P19}

\section{EDS HETEROGENEITY: A FORAY INTO THE DIAGNOSTIC DILEMMA}

Howard Meng1, L Pink, D Paneduro, A Gordon

${ }^{1}$ Wasser Pain Management Centre, Mount Sinai Hospital, Toronto,

Ontario

POSTER AIM: The purpose of this chart review is to better identify symptoms of Ehlers Danlos Syndrome Hypermobility Type (EDS HT) in order to inform the development of a diagnostic questionnaire for future research and improve treatment options available for this condition.

METHODS: Forty-eight patient charts were reviewed retrospectively. Patients suspected of having EDS HT were diagnosed based on history and clinical signs and symptoms. Demographic information, hallmark characteristics of EDS, and EDS-associated symptoms and diagnoses were extracted from patient charts.

RESULTS: Ninety-four \% of patients presenting to the clinic with EDS were female and most have not yet received genetic testing $(85.4 \%)$. The majority of patients did not have a formal documented Beighton score (64.6\%) although hypermobility was common (88.2\%) among those who had a score $(35.4 \%)$. Some of the most frequently reported symptoms included gastrointestinal $(77.1 \%)$, cardiac $(35.4 \%)$, musculoskeletal (100\%), cutaneous $(37.5 \%)$, bladder $(52.1 \%)$, and vulvovaginal $(41.7 \%)$. Additional prevalent symptoms included psychiatric (70.8\%), fatigue (64.6\%), persistent headaches (79.2\%), and sleep disturbances (85.4\%). Postural orthostatic tachycardia syndrome $(8.3 \%)$ and joint dislocations (29.2\%) were less commonly identified.

CONCLUSIONS: Although EDS HT is characterized by the absence of typical cutaneous manifestations, these symptoms were common in our sample. While one of the primary sources of chronic joint pain is thought to be recurrent joint dislocations, these symptoms were not commonly reported. This study highlights the challenge of diagnosing EDS HT and will guide future prospective research initiatives and the development of a diagnostic tool to facilitate early detection and prompt referral to specialists.

\section{P20}

\section{CAN A NURSE INITIATED PAIN PROTOCOL (NIPP) IMPROVE PAIN MANAGEMENT IN THE ED?}

Janice Muir ${ }^{1}$, G Ashly, A Dewar, P Munro, B Parappilly, A Kinkade, J Marsden, E Grafstein

${ }^{1}$ Providence Health Care, University of British Columbia, Vancouver, British Columbia

POSTER AIM: To determine the impact of a Nurse Initiated Pain Protocol (NIPP) on Emergency Department (ED) patient's pain outcomes and pain documentation.

METHODS: A pre and post intervention comparison study using retrospective chart audits followed by a focus group interview.

RESULTS: Charts of 150 ED patients CTAS level II to V from May 2012 were compared to 150 charts from May 2013 after a NIPP was implemented. The mean wait time to first analgesic in 2009 was 102 minutes. Between May 2012 and May 2013 the mean wait time dropped from 91.5 to 85.6 minutes. Within this sample, the NIPP was used only 4 times but the mean wait time dropped to 51.8 minutes. In 2013 73\% of ED patients with pain received analgesics an improvement from the $45 \%$ of ED patients who received analgesics in the 2009 chart audit. The focus group identified system issues, culture of the ED, NIPP limitations, the lack of confidence of nurses regarding pain assessment, and inadequate pain reassessment as themes.

CONCLUSIONS: The NIPP can decrease the wait time for first analgesic, improve comfort and decrease stress on patients, families and health care professionals. Nurses, and other health care professionals need to understand the importance of the use of balanced analgesia, pain assessment and reassessment. Patients and families need to understand the value of acetaminophen and an NSAID like ibuprofen, in lessening the need for stronger analgesics. And finally, system changes to promote the use of a NIPP in the 
ED need to be made to improve the ease of accessibility to the NIPP. It should be noted that since the completion of this research the Vancouver Coastal Health Region has initiated a regional Nurse Initiated Pain Protocol for mild/moderate pain for all ED's and urgent care settings in 2014.

\section{P21}

COMPARING APPLES TO ORANGES? APPROPRIATENESS OF THE USE OF THE FACES PAIN SCALE - REVISED AND A NUMERICAL RATING SCALE FOR PARENTS' RATINGS OF THEIR CHILDREN'S PAIN

\section{Katelynn E Boerner ${ }^{1}$, KA Birnie, M Noel, L Caes, M Petter,} CT Chambers

${ }^{1}$ Dalhousie University, IWK Health Centre, Halifax, Nova Scotia

POSTER AIM: The Faces Pain Scale-Revised (FPS-R) is a well-validated self-report measure of children's pain, depicting faces gradually increasing in pain expression. Although not validated for this purpose, the FPS-R is often used to obtain parent ratings of children's pain. This has been criticized for potential confusion between observed facial expressions of pain and the child's subjective pain intensity. This study explored accuracy of parental pain estimates comparing the FPS-R and an 11-point numerical rating scale (NRS).

METHODS: Healthy children ( $n=48,8-14$ years old) completed the cold pressor task while their parent observed. Parents provided ratings of catastrophizing about their child's pain. Parents (FPS-R and NRS) and children (FPS-R) independently rated the child's pain. Accuracy was defined as parents choosing the same FPS-R face as the child, or the number on the NRS $( \pm 1)$ that corresponded with the child's FPS-R rating.

RESULTS: Parental accuracy was slightly greater with the FPS-R (45.8\%) than the NRS (41.7\%). Underestimation of children's pain was the most frequent error with the FPS-R $(31.3 \%)$ and overestimation the most frequent error with the NRS (33.3\%). Parental state pain catastrophizing was correlated with parent pain ratings, but did not predict congruency with child ratings for the FPS- $\mathrm{R}$ (Block $\left(\chi^{2}(1, \mathrm{~N}=47)=0.45, \mathrm{p}=0.50\right)$ or the NRS (Block $(\chi 2(1, \mathrm{~N}=47)=0.43, \mathrm{p}=0.51)$.

CONCLUSIONS: Although both parent measures resulted in different errors, they were comparable in accuracy for rating children's pain. The FPS-R has an advantage with ease of comparison with children's own ratings and appropriateness with young children. Advantages and disadvantages of the FPS-R and NRS will be discussed.

\section{P22}

PROCEDURAL PAIN IN NEONATAL UNITS IN KENYA A PROSPECTIVE STUDY

O'Brien M Kyololo $^{1}$, BJ Stevens, D Gastaldo, J Songok

${ }^{1}$ University of Toronto, Toronto, Ontario; Moi University, Eldoret, Kenya

POSTER AIM: To (a) determine the nature and frequency of painful procedures during the first seven days of admission, and management practices implemented, and (b) explore the factors that influence the nature and frequency of painful procedures in two neonatal units in Kenya.

METHODS: A validated checklist was used to document painful procedures performed on neonates during the first seven days of admission to two neonatal units in Kenya. Descriptive statistics were computed for demographic characteristics and the nature and frequency of (a) painful procedures and (b) analgesia administered. Linear regression modelling was used to explore the influence of neonatal, situational, and organizational factors on the nature and frequency of painful procedures and analgesia use. $\mathrm{P}$-value $<0.05$ was specified as statistically significant.

RESULTS: Two hundred neonates, 100 from each unit, were followed between June and September 2014. Over the hospitalisation period (mean 3.56 days, SD 1.95; range 1-7 days), the neonates underwent a total of 1479 painful procedures, 1084 skin-breaking and 395 non skin-breaking, for an average of 1.34 (SD 1.57; range 0-12) procedures for each day of hospitalisation. Most procedures were performed during the first two days of life (Mean =2.27, SD 1.69; range 0-12). The three most prevalent procedures, intramuscular injection, venepuncture for bloodwork, and peripheral venous cannula insertion, constituted nearly two thirds of the total procedures $(n=932,63 \%)$. Birth weight, gestational age, place of birth, and severity of illness did not influence nature and frequency of painful procedures. Neonates that were hospitalized in a lower level (level I) neonatal unit were likely to experience less number of painful procedures than those in a higher level (level II) unit $\left(\mathrm{I}^{2}=-0.522,95 \% \mathrm{CI}-0.891\right.$ to $-0.152 \mathrm{p}=0.006)$.

CONCLUSIONS: Neonates undergo numerous skin-breaking and nonskin-breaking procedures that are painful during the first days of life. Most of the procedures are moderate to severely invasive and none are accompanied by analgesia. A multi-sectoral approach is required to improve the sub-optimal pain management in neonatal units in Kenya.

\section{P23}

\section{META-ANALYSIS OF PAIN, HEALTH AND PSYCHOLOGICAL OUTCOMES IN CHILDREN OF PARENTS WITH CHRONIC PAIN}

Kristen Higgins ${ }^{1}$, KA Birnie, CT Chambers, AC Wilson, L Caes, AJ Clark, M Lynch, J Stinson

${ }^{1}$ Dalhousie University, IWK Health Centre, Halifax, Nova Scotia

POSTER AIM: Children of parents with chronic pain may have poorer outcomes compared to children of healthy parents. This review builds on previous work by using a broader search strategy, being more inclusive of different study designs and including a meta-analysis.

METHODS: A systematic search of electronic databases was conducted for published articles in English examining pain, health, psychological, or family outcomes in offspring of parents with chronic pain.

RESULTS: Fifty-four eligible articles were identified, with 18 quantitative $(n=12)$ or population-based $(n=6)$ studies reporting data appropriate for meta-analysis. They include offspring from birth to adulthood and represent parents with varying chronic pain diagnoses (e.g., mixed pain samples, headaches, back pain). Increased pain complaints were found in children of mothers $(Z=5.55, p<0.00001)$, fathers $(Z=2.46, p=0.01)$, or both parents with chronic pain $(Z=4.06, p<0.0001)$. Children of parents with chronic pain had greater externalizing $(\mathrm{SMD}=0.66,95 \% \mathrm{CI}[0.35,0.98]$, $\mathrm{Z}=4.10, \mathrm{p}<0.00001)$ and internalizing problems $(\mathrm{SMD}=0.73,95 \% \mathrm{CI}$ $[0.50,0.97], Z=6.15, p<0.00001)$, and poorer family outcomes $(\mathrm{SMD}=0.49$, $95 \%$ CI $[0.18,0.80], Z=3.11, p=0.002)$. No significant group differences were found on social competence $(Z=1.06, p=0.29)$ or teacher-reported externalizing problems $(Z=1.64, p=0.10)$. Newborns of mothers with chronic pain were more likely to have adverse birth outcomes, including low birthweight, preterm delivery, caesarian, etc.

CONCLUSIONS: Children of parents with chronic pain are at increased risk for poorer pain, health, and psychological outcomes compared to children of healthy parents.

\section{P24}

INITIAL CLINICAL RESPONSE TO METHADONE IN CANCER PAIN PATIENTS

Jordi Perez ${ }^{1}$, S Olivier, J Borniard

${ }^{1}$ Alan Edwards Pain Management Unit, Montreal General Hospital, Montreal, Quebec

POSTER AIM:

1) To describe the population of ambulatory patients seen at McGill University Health Centre's Cancer Pain Clinic that received Methadone as analgesic.

2) To present the outcomes within the first five days of treatment.

METHODS: Retrospective chart review of patients' initial response to methadone. Research variables:

1) Demographic: Age, gender, cancer status, quality of pain, average pain severity

2) Methadone initiation: aim of treatment (full rotation vs. coadjuvant), test dose done in clinic (yes/no), initial dose (total daily $\mathrm{mg}$ )

3) Initial response: short-acting opioids consumption, perceived efficacy, side effects and action taken after phone assessment

RESULTS:

1) Demographics: Gender: $59 \% \sigma^{\pi}-41 \%$ \%; Age: $64.4 \pm 12.8$ years: Cancer Status 30.1\% local $/ 59.5 \%$ advanced; Neuropathic pain? $69 \%$ yes $/ 26 \%$ no; Average pain severity $7.44 \pm 1.95$ 
2) Initial methadone trial: Aim of treatment: $76.2 \%$ rotation $/ 23.9 \%$ coadjuvant; Test dose given in clinic? In $21.4 \%$ of cases; initial dose of methadone: $8.7 \pm 7.06 \mathrm{mg} /$ day

3) Initial response: Efficacious? $64.3 \%$ yes, $26.2 \%$ no, 9.5\% unclear; short acting opioid consumption: $45 \%$ decreased, $23.8 \%$ unchanged, $9.5 \%$ increased; side effects: $69 \%$ none $/ 31 \% \geq 1$ (drowsiness); action taken after consultation: Discontinuation: $2.4 \%$, Dose decreased: $16.7 \%$, dose unchanged: $33.3 \%$, dose increased $45.2 \%$

CONCLUSIONS: During the first week of treatment, ambulatory patients receiving methadone reported good effect, less or unchanged opioid consumption and little side effects. Further data sub analysis and a correlational investigation will be presented during the conference.

\section{P25}

PREDICTING CHILD PAIN OUTCOMES FROM CHILD AND PARENT PAIN CATASTROPHIZING, ANXIETY AND DISTRESS Kathryn A Birnie ${ }^{1}$, CT Chambers, J Chorney, CV Fernandez, PJ McGrath

${ }^{1}$ Dalhousie University, IWK Health Centre, Halifax, Nova Scotia

POSTER AIM: Catastrophic thinking by parents or children is known to influence children's pain; however, little is known about how both parents and children's pain catastrophizing and anxiety/distress interact to influence children's pain responding.

METHODS: 172 children (89 girls) aged 8-12 years $(M=10.02 ; S D=1.38)$ and a parent (136 mothers) completed measures of trait and state pain catastrophizing (PCS-C; PCS-P), trait anxiety (SCARED; BAI), and state distress. After interacting while the child completed the cold pressor task, parents and children rated the child's pain intensity (FPS-R) and unpleasantness (11-point NRS). A series of step-wise hierarchical multiple regressions were used with child variables entered first for self-reported pain outcomes and parent variables entered first for parent-rated pain outcomes. RESULTS: Child variables accounted for $37.8 \%$ of variance in pain intensity $(\mathrm{R}=0.62 ; \mathrm{F}(4,167)=25.39, \mathrm{p}<0.01)$ and $51.0 \%$ of variance in pain unpleasantness $(\mathrm{R}=0.71 ; \mathrm{F}(4,167)=43.46, \mathrm{p}<0.01)$, with no added impact of parent variables to either model. Only child state pain catastrophizing contributed significantly to these predictions $(\mathrm{p}<0.01)$. Parent variables accounted for $22.7 \%$ of variance in parent-rated pain intensity $(R=0.478$; $\mathrm{F}(5,166)=9.77, \mathrm{p}<0.01)$ and $30.5 \%$ of variance in pain unpleasantness $(\mathrm{R}=0.552 ; \mathrm{F}(5 ; 166)=14.55, \mathrm{p}<0.01)$. Only parent state pain catastrophizing $(\mathrm{p}<0.01)$ remained a significant predictor once child variables were entered. Child variables accounted for an additional $8.0 \%$ of variance in parent-rated pain intensity and $6.1 \%$ of variance in parent-rated pain unpleasantness, driven by children's state pain catastrophizing $(\mathrm{p}<0.01)$.

CONCLUSIONS: Children's state pain catastrophizing was the strongest predictor of child pain outcomes and contributed significantly above parental characteristics to parent-rated pain outcomes.

\footnotetext{
P26

DOES CULTURE INFLUENCE THE EXPERIENCE OF NECK PAIN?

Howard Vernon ${ }^{1}$, W Goodman, RR Weaver, M Monticone, G Guzy, M Bakhtadze, M Bednarikova, J Crawford, N Kesiktas ${ }^{1}$ Canadian Memorial Chiropractic College, Toronto, Ontario

POSTER AIM: According to the US Burden of Disease Collaborations, neck pain is the 4th leading cause of years lived with disability in the US. This status remains unchanged from 1990 to 2010. The findings of the WHO Global Burden of Disease Report indicate that, in 1990, neck pain was the 25th leading cause of global-adjusted disability years, while, in 2010, it rose to the 21st position (above, eg., lung cancer, other musculoskeletal disorders). Given this world-wide prevalence, it could be assumed that neck pain is experienced similarly by most people globally. It has been shown that culture, ethnicity and race importantly influence the access and provision of health care within Western countries creating disparities amongst different groups of people within these countries. With respect to neck pain more specifically, there are a number of studies which report differences in the attitudes toward and care of people with whiplash injuries. These studies purport to show that culture is an important determinant in the...
}

METHODS: Ten subjects with chronic neck pain, male or female, ages 18-65 were recruited in clinics in each national sites: Cracow, Poland; Ankara, Turkey; Moscow, Russia; Milan, Italy; Prague, Czech Republic and Toronto, Canada. Exclusion criteria were: pain lasting less than 6 weeks; presence of arm pain with neck pain (cervical radiculopathy); diagnosis of any pathology other than mechanical neck pain associated with joint or muscular dysfunction. While a mixed-methods approach was used which included interviews for the qualitiative data, only the quantitative data are presented here. All subjects completed a demographic and clinical baseline form as well as the NRS-101 for pain severity, the Neck Disability Index for self-rated disability, the McGill Pain Questionnaire for pain quality and severity and the NeckPix instrument for assessing fear-avoidance beliefs related to neck pain. A demographic and clinical baseline form was completed by all subjects except those in the Turkish site. All measures were normal...

RESULTS: Significant correlations were found among all four pain measures, with the strongest being between MPQ and NDI $(r=0.798$, $p$-value $=$ 0.000). However MPQ data were missing for all records from Poland. The ANOVA tests found significant differences for pain measures among the sites. (For Pain Severity, p-value = 0.014; for NDI, p-value = 0.004; for MPQ, $\mathrm{p}$-value $=0.005$; and for NeckPix, $\mathrm{p}$-value $=0.016$.) Regression models were all significant for NDI, MPQ, and NeckPix, respectively, using dummy variables for country, and controlling for pain severity. (All models' p-values $<0.02$.) In those models, significant country coefficients for elevated pain measures were: for NDI, Russia ( $\mathrm{p}$-value $=0.028$ ); for MPQ, Russia ( $\mathrm{p}$-value = 0.032); and for NeckPix, Poland ( $p$-value = 0.026). For MPQ, Turkey was also found to be associated with decreasing MPQ ( $p$-value $=0.036$ ). Duration of pain (in months) was the only demographic factor tentatively identified as possibly influencing the above results.

CONCLUSIONS: The choice of quantitative instruments applied in this study was successful in demonstrating distinctions amongst the cultures investigated. While the sample size at each site was small, these findings give preliminary support to the notion that culture does have some influence on the experience of neck pain. Further research into this phenomenon is warranted and could provide a basis for more culturally-grounded approaches to the assessment and management of neck pain.

\section{P27}

INVESTIGATING THE ANALGESIC EFFECT FOR STRUCTURAL DERIVATIVES OF THE NOVEL ANALGESIC ISOVALINE

\section{Timothy Fung ${ }^{1}$, E Puil, BA MacLeod}

${ }^{1}$ University of British Columbia, Vancouver, British Columbia

POSTER AIM: The purpose of this study was to establish a structure activity relationship for the analgesic effect of R-isovaline in order to create new analgesics with better therapeutic ratios than current analgesics. A range of compounds with representative structural modifications were made to the $\mathrm{R}$-groups of $\mathrm{R}$-isovaline and will be tested to determine their analgesic efficacy and side effects.

METHODS: The analgesic effects of the different compounds were assessed using the formalin foot assay. Saline or $500 \mathrm{mg} / \mathrm{kg}$ compounds were administered intraperitoneally (i.p.) 15 minutes prior to the administration of $5 \%$ formalin into the right hind paw of the mouse (except R-isovaline which was administered 45 minutes prior). Mouse paw licking over an hour was recorded on video and subsequently analyzed by a blinded observer.

RESULTS: The total time the mouse spent licking the affected paw between 15-45 minutes after the administration of formalin was compared between treatment and saline. Unpaired t-tests found a statistically significant difference $(\mathrm{p}<0.05)$ in $\mathrm{R}$-isovaline $(210.5 \pm 28.4 \mathrm{~s}, \mathrm{n}=6)$, S-isovaline $(208.6 \pm 27.9 \mathrm{~s}, \mathrm{n}=5)$, and 1-amino-1-cyclobutane carboxylic acid $(195.4 \pm 43.1 \mathrm{~s}, \mathrm{n}=5)$ compared to their respective saline controls $(318.3 \pm$ $28.4 \mathrm{~s}, \mathrm{n}=6,293.3 \pm 21.3 \mathrm{~s}, \mathrm{n}=6$, and $343.8 \pm 40.4 \mathrm{~s}, \mathrm{n}=5$ ). No statistically significant difference was found between saline and $R-\alpha$-methylphenylalanine, $\mathrm{R}$ - $\alpha$-propargylalanine, and $\mathrm{R}$ - $\alpha$-allylalanine.

CONCLUSIONS: This study has identified two structural relatives of R-isovaline, S-isovaline and 1-amino-1-cyclobutane carboxylic acid, which have an analgesic effect. No analgesic effect was observed for R- $\alpha$ methylphenylalanine, $\mathrm{R}$ - $\alpha$-propargylalanine, and R- $\alpha$-allylalanine. 


\section{P28}

REPORTING OF IMMPACT-RECOMMENDED CORE OUTCOME DOMAINS AMONG TRIALS ASSESSING OPIOIDS FOR CHRONIC NON-CANCER PAIN

Sohail Mulla ${ }^{1}$, LC Lopes, S Schandelmaier, M Kamaleldin, S Hsu, JJ Riva, PO Vandvik, L Tsoi, T Lam, S Ebrahim, BC Johnston,

L Olivieri, L Montoya, R Kunz, A Scheidecker, DN Buckley,

DI Sessler, GH Guyatt, JW Busse

${ }^{1}$ McMaster University, Hamilton, Ontario

POSTER AIM: The Initiative on Methods, Measurement, and Pain Assessment in Clinical Trials (IMMPACT) has recommended that clinical trialists evaluating treatments for chronic pain should consider reporting nine patient-important outcome domains. We examined the extent to which clinical trials evaluating the effect of opioids for chronic non-cancer pain (CNCP) report each of the outcome domains recommended by IMMPACT.

METHODS: We systematically searched electronic databases for Englishlanguage studies that randomized patients with $\mathrm{CNCP}$ to receive an opioid or a non-opioid control. In duplicate and independently, reviewers established the eligibility of each identified study, and recorded all reported outcome domains from eligible trials. We conducted a priori regression analyses to explore factors that may be associated with reporting the core outcome domains recommended by IMMPACT.

RESULTS: Among 165 eligible trials, reporting of IMMPACT recommended outcome domains was variable. Recently published trials were more likely to report the effect of treatment on physical functioning, emotional functioning, role functioning, sleep and fatigue, and participant disposition. Trials for which the corresponding author was from North America were more likely to report treatment effects on physical functioning, and participant ratings of improvement and satisfaction with treatment. Trials published in journals with higher impact factors were more likely to report treatment effects on emotional function, but less likely to report participant ratings of improvement and satisfaction with treatment.

CONCLUSIONS: Most IMMPACT domains showed an increased rate of reporting over time, although many patient-important outcome domains remained unreported by over half of all trials evaluating the effects of opioids for CNCP.

\section{P29 \\ PREDICTORS OF SUCCESSFULLY MANAGING DEPRESSIVE SYMPTOMS AMONG CHRONIC PAIN PATIENTS}

Sohail Mulla ${ }^{1}$, EG Hapidou, SM Kallyth, DN Buckley, JW Busse ${ }^{1}$ McMaster University, Hamilton, Ontario

POSTER AIM: Chronic pain patients often present with depressive symptoms or depression is a co-morbid disorder for individuals with chronic pain. The Chronic Pain Management Unit (CPMU) within Hamilton Health Sciences (Ontario, Canada) offers a four-week, multimodal, interdisciplinary, group-based treatment program for chronic noncancer pain patients. The purpose of our study was to explore patient characteristics collected at admission into the CPMU that are associated with successfully managing their depressive symptomology.

METHODS: We examined all patient records that the CPMU has electronically maintained since 2006. We defined successful management of depressive symptoms as a minimal clinically important difference (MCID) on the Centre for Epidemiological Studies-Depression (CES-D) scale from discharge to admission. We conducted a priori multiple logistic regression analyses to explore factors that may be associated with successful management.

RESULTS: A total of 896 patients attended the CPMU program from January, 2006 to January, 2014. On average, patients were 44.0 years old and suffered from pain for 4.5 years. Typical patients were female $(51.7 \%)$, born in Canada (78.1\%), married (65.5\%), and unemployed (64.0\%). Of 686 patients with complete outcome information, 399 (58.2\%) successfully managed their depressive symptoms. Our complete case analysis $(n=500)$ found that the odds of achieving an MCID on the CES-D are 1.273 times greater in older patients than in younger patients $(95 \%$ confidence interval: $1.049-1.546 ; \mathrm{p}=0.015)$. We did not find any statistically significant associations with other admission variables.
CONCLUSIONS: Older patients are more likely to successfully manage their depressive symptoms than younger patients.

\section{P30}

DEVELOPMENT OF A PAINLESS INJECTION DEVICE APPLYING PRESSURE, VIBRATION AND TEMPERATURE William McKay ${ }^{1}$, R Gusztak, T Bolton, A Frost, A Wang, B Kushneriuk, J Cowen, D Chen

${ }^{1}$ University of Saskatchewan, Saskatoon, Saskatchewan

POSTER AIM: Pain and distress are associated with needle sticks. We quantify combined pressure $(\mathrm{P})$, vibration $(\mathrm{V})$, and warming (T: temperature) applied to the skin to decrease needle pain. P, V, and T were studied independently previously; this study combines their optimal levels.

METHODS: With Ethics approval, 21 healthy adults were taught the verbal rating scale for pain, (0: no pain; 10: the worst pain possible), and seated so that the investigator could see the monitor but the subject could not. An Electronic Von Frey Hair (EVFH) was applied to the deltoid, slowly increasing force until the subject rated a 3/10 pain. Baseline measurements were completed twice on both arms. A force transducer attached to a copper foot plate with a hole for the EVFH measured P. The optimal P (400 torr) was applied for $20 \mathrm{~s}$ prior to and during the application of the EVFH until 3/10 pain was achieved. This was repeated on alternating shoulders. As with $\mathrm{P}$, optimal V (frequency $100 \mathrm{~Hz}$; amplitude $130 \hat{A}_{\mu}$ ) and T were applied using a variable amplitude vibrator and thermal stimulator and thermode attached to the footplate. The protocol was repeated with simultaneous $P \& V$, then with simultaneous P\&V\&T. Repeated-measures ANOVA was used to compare EVFH forces for the baseline and combined modalities.

RESULTS: All combinations were significantly better than baseline $(\mathrm{P}<0.001)$; $\mathrm{P} \& \mathrm{~V}$ and $\mathrm{P} \& \mathrm{~V} \& \mathrm{~T}$ were better than $\mathrm{P}$ alone $(\mathrm{P}<0.005)$, but not different from each other.

CONCLUSIONS: Combining optimal pressure, vibration, and warming applied to the skin decreases needle pain.

\section{P31}

OBSERVATIONAL TRIAL OF QIGONG AS A COMPLEMENTARY PRACTICE IN A CHRONIC PAIN PROGRAM

Meghan Pike ${ }^{1}$, J Sawynok, M Lynch, AJ Clark, D Marcon

${ }^{1}$ Dalhousie University, Halifax, Nova Scotia

POSTER AIM: Chaoyi Fanhuan Qigong (CFQ) has produced benefit in fibromyalgia. In this study, the effect of CFQ on pain, mood, quality of life, sleep and sense of control are measured.

METHODS: Participants attended a 2-hour class weekly for 6-7 weeks. Demographics, medical history, Health Locus of Control, Attitudes Toward Complementary and Alternative Therapies, Brief Pain Inventory, Profile of Moods State, Satisfaction and SF-12V2 Quality of Life Survey were collected at baseline and after 6-7 weeks. ANOVA was used to compare outcomes to baseline. A thematic analysis was used to evaluate qualitative information.

RESULTS: The first cohort ( $\mathrm{n}=17$ ) consisted of $12 / 5$ female/male subjects with chronic pain, average age 56.7. Subjects attended an average of 6.6 CFQ classes and averaged 70 minutes per day of self-practice. At six weeks compared to baseline no significant change in scores for average pain level, mood or quality of life were found. A statistically significant decrease in pain interference scores was found and six patients reported a higher internal Health Locus of Control score. Qualitative comments indicated improvements in asthma, digestion and quality of sleep.

CONCLUSIONS: This preliminary analysis revealed a statistically significant decrease in pain interference scores after six weeks of CFQ practice. CFQ is a practice that takes time to learn, changes in average pain score, mood and function scores may become significant after these patients are enrolled for a longer period. Improvements in internal locus of control suggest CFQ practice may provide some patients with improved sense of control over health. 
P32

PREVALENCE OF OPIOID USE AND CHARACTERISTICS ASSOCIATED WITH OPIOID USE PROFILE AMONG CHRONIC NON-CANCER PAIN PATIENTS ATTENDING A MULTIDISCIPLINARY PAIN TREATMENT FACILITY: A QUEBEC PAIN REGISTRY STUDY

Hichem Saidi ${ }^{1}$, G Pagé, M Ware, M Choinière

${ }^{1}$ Centre de recherche du centre hospitalier de l'universite de Montreal, Montreal, Quebec

POSTER AIM: The goals of the present study were to:

1) Determine the proportion of patients with chronic non-cancer pain (CNCP) who (a) were using opioids before initiating treatment at a multidisciplinary pain treatment facility (MPTF) and (b) started using opioids following this initial visit;

2) Characterize the opioid use profile (OUP) of CNCP patients over the first year of attending a MPTF; and

3) Identify baseline characteristics associated with OUP.

METHODS: Participants were 8,521 patients (mean age $=53.24(\mathrm{SD}=14.4)$; female $=58.3 \%$ ) enrolled in the Quebec Pain Registry since 2008. Patients completed a series of questionnaires before initiating treatment and at 6 months; data is also available on a subset of patients $(n=1,955)$ at 12 months. Univariate multinomial logistic regressions (MLR) followed by multivariable MLR were used to examine the association between biopsychosocial variables and OUP membership.

RESULTS: Thirty-six percent of patients were already using opioids before the initial visit and $15 \%$ initiated opioid therapy following the initial visit. Forty-three percent of patients were classified as non-users (not using opioids), $40 \%$ as non-persistent users (stopped using opioids during the study), and $17 \%$ as persistent users (using opioids throughout the study). The multivariable MLR showed that depression, pain frequency, quality of life, alcohol and cigarette consumption were significantly associated with OUP membership $\left(\chi^{2}[38]=199.66, p<0.001\right)$.

CONCLUSIONS: A significant proportion of CNCP patients use opioids and different biopsychosocial characteristics are associated with OUP. Future research should examine the association between these characteristics and opioid treatment effectiveness.

\section{P33}

LONG-TERM EFFECTIVENESS OF OPIOIDS AMONG CHRONIC NON-CANCER PAIN PATIENTS ATTENDING A MULTIDISCIPLINARY PAIN TREATMENT FACILITY: A QUEBEC PAIN REGISTRY STUDY

\section{Hichem Saidi ${ }^{1}$, G Pagé, M Ware, $M$ Choinière}

${ }^{1}$ Centre de recherche du centre hospitalier de l'universite de Montreal, Montreal, Quebec

POSTER AIM: The goal of the present study was to examine the longterm effectiveness of opioids in terms of reducing pain intensity, pain interference and improving physical (pQofL) and mental (mQofL) healthrelated quality of life of patients with chronic non-cancer pain (CNCP). METHODS: Participants were 1,955 patients (mean age $=53.07(\mathrm{SD}=$ $14.51)$; female $=58.3 \%$ ) enrolled in the QPR (2008-2012) and who completed a series of questionnaires before initiating treatment at a multidisciplinary pain treatment facility (MPTF) as well as 6 and 12 months thereafter. Linear mixed models were used to examine whether sex, age, and opioid use profile (OUP) (non-users, non-persistent users, and persistent users) predicted pain intensity, pain interference, pQofL and mQofL over a one-year period.

RESULTS: In all four models, sex, age and OUP significantly predicted pain intensity, pain interference, pQofL and mQofL (all p-values $<0.05)$. Compared to non-users, persistent opioid users had significantly higher levels of pain intensity and interference and worse pQofL and mQofL across time. A significant interaction between sex and age $(p<0.05)$ showed that among older patients, males had higher levels of pain intensity interference compared to females. A significant sex-ageOUP interaction $(\mathrm{p}<0.05)$ was found for $\mathrm{pQofL}$ and $\mathrm{mQofL}$ such that among persistent users, young males had a better QofL compared to young females.
CONCLUSIONS: These results suggest that long-term use of opioids does not improve patients' pain experience and raise an important question about the role opioids should play in the therapeutic arsenal aimed at managing CNCP.

\section{P34}

ARE BOYS' AND GIRLS' PAIN JUDGED THE SAME? THE IMPACT OF CAREGIVER JUDGMENT OF CHILDREN'S PAIN Meghan Schinkel ${ }^{1}$, KE Boerner, CT Chambers, CM McMurtry ${ }^{1}$ Dalhousie University, Halifax, Nova Scotia

POSTER AIM: Low levels of agreement between parent and child reports of acute pain are well documented. There is some evidence that fathers may be more accurate judges of child pain than mothers. The current study examined the influence of caregiver and child sex on caregivers' judgments of children's pain during venipuncture.

METHODS: 133 caregivers ( 76 female, 57 male) viewed twenty 10 -second video clips of unrelated children ( 10 boys, 10 girls), aged 5-10 years, undergoing venipuncture, and then rated the child's pain intensity using the Faces Pain Scale-Revised (FPS-R). Caregivers' ratings were compared to the child's self-reported FPS-R ratings of pain intensity (exact agreement was considered an accurate judgment).

RESULTS: The impact of caregiver and child sex on caregiver judgment accuracy was analyzed using a $2 \times 2$ mixed analysis of variance (ANOVA). A significant main effect of child sex was discovered, $F(1,131)=39.88$, $p<0.001$, partial $\eta 2=0.23$, with caregivers accurately judging boys' pain significantly more often than girls'. No main effect of parent sex, or parent by child sex interactions emerged. Caregivers underestimated both boys' $(\mathrm{M}=58.36 \%, \mathrm{SD}=22.39)$ and girls' pain $(\mathrm{M}=64.55 \%, \mathrm{SD}=19.15)$ more frequently than they overestimated or accurately judged children's pain.

CONCLUSIONS: The findings suggest that child sex may impact caregiver's assessments of children's pain, with caregivers accurately judging boys' pain more frequently. Further, parents have a tendency to underestimate children's pain, highlighting the importance of also considering children's self-report ratings of pain in clinical settings.

\section{P35}

AN ANALYSIS OF CHILD SELF-REGULATION ACROSS PAINFUL AND NON-PAINFUL CONTEXTS: THE ROLE OF BEHAVIOURAL INHIBITION

Masa Calic ${ }^{1}$, R Pillai Riddell, H Garfield, S Greenberg

1York University, Toronto, Ontario

POSTER AIM: The study explores the relationship between self-regulation competency during a delay of gratification task (waiting for a marshmallow, a non-painful positive stimuli), and during an immunization appointment (waiting for a needle, a painful negative stimuli). The role of behavioural inhibition was hypothesized to mediate the relationship of self-regulation in both contexts.

METHODS: A subsample of 64 preschool children (age ranged 4 to 6 years) was obtained from a larger cohort (The OUCH Cohort; Pillai Riddell et al., 2011). Children and their parents were videotaped during the child's preschool immunization appointment and subsequently participated in a follow-up laboratory assessment. Among the tasks, children participated in the well-known Marshmallow Task (waiting for a marshmallow in order to earn two marshmallows; Mischel, Shoda, \& Rodriguez, 1989) and a well-validated measure of behavioural inhibition (Statue Subtest of NEPSY-II; Brooks, Sherman, \& Strauss, 2009). Self-regulation during the Marshmallow Task was defined as latency to first eat the marshmallow, while the total pre-needle behavioural distress score (FLACC, Merkel et al., 1996) operationalized self-regulation in a pain context. Multiple regression mediation and correlational analyses were conducted. RESULTS: The proposed mediation relationship was not supported; selfregulation in the marshmallow and immunization context was not explained by the level of behavioural inhibition. A significant positive correlation was found between the child's performance on the marshmallow and the behavioural inhibition task.

CONCLUSIONS: According to this analysis, behavioural inhibition did not mediate the relationship between a child's self-regulation in the 
marshmallow and immunization contexts. Moreover, self-regulation in these two contexts was not related, and behavioural inhibition was only related to self-regulation in the positive distress context.

\section{REFERENCES}

Brooks, B. L., Sherman, E. M., \& Strauss, E. NEPSY-II: A developmental neuropsychological assessment. Child Neuropsychology 2009;16(1):80-101.

Merkel, S. I., Voepel-Lewis, T., Shayevitz, J. R., \& Malviya, S. The FLACC: A behavioral scale for scoring postoperative pain in young children. Pediatric Nursing 1996;23(3):29.

Mischel, W., Shoda, Y., \& Rodriguez, M. Delay of gratification in Children. Science 1989;244(4907):933-938.

Pillai Riddell, R., Campbell, L., Flora, D. B., Racine, N., Din Osmun, L., Garfield, H., \& Greenberg, S. The relationship between caregiver sensitivity and infant pain behaviors across the first year of life. Pain, 2011;152(12):2819-2826

\section{P36}

\section{GENETICS AND MIGRAINE: STUDY ON THE} RELATIONSHIP BETWEEN COMT AND MIGRAINE

Tobore Onojighofia ${ }^{1}$, B Meshkin, D Schwarz, B Akindele, J Hubbard, Mary Knauer

${ }^{1}$ Proove Biosciences

POSTER AIM: The objective of this study is to investigate the role of the COMT polymorphism in genetic susceptibility to migraine.

METHODS: 49 subjects across 8 clinical research sites in the US. 24 diagnosed with migraine (ICD9 codes 346 series), mean age (44), males (2), females (22), and 25 controls matched for race, gender and age. Subjects were genotyped using Taqman ${ }^{\circledR}$ SNP Genotyping Assays (Life Technologies, Carlsbad, CA). It consists of a panel of 12 single nucleotide polymorphisms (SNPs) in genes encoding for proteins expressed in the mesolimbic reward pathway. These genes include: 5HT2a, 5-HTTL, COMT, ANKK1/DRD2, DRD1, DRD4, DAT, DBH, MTHFR, OPRK1, GABA-A receptor gamma2, and OPRM1.

RESULTS: A cross tab analysis using IBM SPSS v21 found significant association between only COMT Val158Met (rs4680) and subjects with migraine (COMT: Dominant Model (G/G vs. G/A-A/A). Pearson chisquare $p=0.011$, Two sided Fisher's exact 0.024). COMT wild type $(G / G)$ showed higher prevalence in subjects with migraine compared to controls. Logistic regression found that COMT G/G is more associated with Migraine compared to the controls ( $p=0.016$ OR 5.923)

CONCLUSIONS: This study suggests that COMT Val158Met (rs4680) may play a role in genetic predisposition to migraine. Findings in this study will hopefully help improve understanding on the role of genetics in migraine.

\section{P37}

RISK OF OPIOID ABUSE AND BIOPSYCHOSOCIAL CHARACTERISTICS ASSOCIATED WITH THIS RISK AMONG CHRONIC PAIN PATIENTS ATTENDING A MULTIDISCIPLINARY PAIN TREATMENT FACILITY

M Gabrielle Pagé ${ }^{1}$, H Saïdi, M Ware, M Choinière

${ }^{1}$ Centre de Recherche du Centre hospitalier de l'Université de Montréal, Montreal, Quebec

POSTER AIM: The objectives of this study were to (1) determine the proportion of new patients attending a multidisciplinary pain treatment facility (MPTF) at risk of opioid abuse and (2) examine biopsychosocial factors associated with this risk.

METHODS: Participants were 4,518 patients ( mean age $=53.5(\mathrm{SD}=15.0)$; female $=57.2 \%$ ) who initiated treatment at a MPTF and enrolled in the Quebec Pain Registry between 2012 and 2014. Patients answered a battery of self-report and nurse-administered questionnaires (pain and related psychosocial constructs, including the Opioid Risk Tool) before initiating treatment. A series of univariate multinomial logistic regression analyses followed by a multivariable logistic regression analysis were used to examine the association between biopsychosocial variables and risk of opioid abuse.

RESULTS: Results showed that $82 \%(\mathrm{n}=3686), 13 \%(\mathrm{n}=586)$ and $5 \%$ $(n=246)$ of patients were at low, moderate and high risk of opioid abuse, respectively. The final multivariable multinomial logistic regression model $\left(\chi^{2}[24]=532.9, p<0.001\right)$ showed that patients at high risk of opioid abuse were older, consumed more alcohol, had a longer pain duration, higher levels of depression, worse sleep quality and worse mental health quality of life compared to patients with low or moderate risk of abuse.

CONCLUSIONS: Almost 20\% of patients had a moderate-high risk of opioid abuse; this risk is associated with several psychosocial characteristics. These results point to the importance of assessing risk of opioid abuse in chronic pain patients and to consider more broadly psychosocial factors that can contribute to this risk.

\section{P38 \\ OPIOID PRESCRIBING IN WORKER'S COMPENSATION CHRONIC PAIN PATIENTS IN NEW BRUNSWICK}

R Thomas Evans ${ }^{1}$, DJ French

${ }^{1}$ The Atlantic Pain Clinic, Moncton, New Brunswick

POSTER AIM: Canada and the United States have the highest rates of inappropriate prescription opioid consumption in the world. The goal of the present study was to examine patterns of opioid prescribing in a large sample of Workers' Compensation (WCB) chronic non-cancer pain patients (CNCP) in New Brunswick, Canada.

METHODS: The medical files of 692 WCB patients, managed by 256 family physicians from across New Brunswick, who were referred to interdisciplinary pain management were analysed for key features of opioid prescribing. These features included, opioid agent, release type, overall Morphine equivalent and adherence to the WHO analgesic stepladder. Patient features known to be associated with increased risk for opiate aberrant behavior were also examined.

RESULTS: Narcotic analgesics were prescribed to $44 \%$ of the sample. Codeine $(50 \%)$ and Oxycodone (35\%) were most commonly prescribed. Despite an average pain chronicity of 115 weeks, immediate release/short acting opiates were prescribed in $70 \%$ of cases. Evidence of adherence to the WHO analgesic stepladder was observed in only $13 \%$ of cases. The Morphine equivalent of these prescriptions was less than 30mg in 65\%. Of patients prescribed opioids, $89 \%$ screened (SISAP/CAGE-AID/COMM) at risk for opiate aberrancy, $45 \%$ had a past history of diagnosed Psychiatric illness, $27 \%$ had a past history of substance abuse and $13 \%$ were currently abusing alcohol or other drugs.

CONCLUSIONS: Despite the availability of opiate treatment guidelines for CNCP, the prescription of narcotic analgesics to WCB chronic pain patients in New Brunswick remains concerning.

\section{P39}

\section{FRAMEWORK FOR CHARACTERIZING SERVICES FOR THE MANAGEMENT OF CHRONIC NON-CANCER PAIN IN GLOBAL CITIES}

$\underline{\text { S Fatima Lakha }}{ }^{1}$, P Pennefather, P Ballantyne, HE Badr, M Agboatwalla, A Mailis

${ }^{1}$ University of Toronto, Toronto, Ontario

POSTER AIM: Interest for pain management strategies seem to be universal. Yet, several international organizations have highlighted failures of pain management delivery globally. Since global cities play important roles in co-locating health care resources and mediating local access to globally recognized standards of care, it is expected that a comparison of pain management services in different global city settings will provide insights into those failures.

METHODS: Semi-structured interviews with key-informants are being used to describe clinical services for chronic pain management in selected global cities. Initially, pain clinics in global cities served by the Eastern Mediterranean Region Office (EMRO) of the WHO are compared to those in Toronto. Key-informants are directors of clinics specializing in pain management and located in those cities. So far pain clinics directors in Kuwait city, Karachi and downtown Toronto have been interviewed. RESULTS: Qualitative analysis of interview records are being used to pragmatically explore barriers to delivery of the internationally accepted standards of care. We have used a combination of Donebedian and Logic model frameworks to organize analysis themes related to structure, process, 
and output elements associated with the surveyed pain clinics. Barriers to output quality are related to infrastructure, services, education and research capacity themes.

CONCLUSIONS: There are no published studies of pain management services in the cities served by EMRO. This study provides direct information on how standard pain management services are delivered in that region. Similarities and differences between Toronto and EMRO cities will be discussed.

\section{P40}

CHRONIC NON-CANCER PAIN MANAGEMENT IN TORONTO: A STUDY OF TEACHING-HOSPITAL BASED SERVICES OFFERED IN CHRONIC PAIN CLINICS

$\underline{\text { S Fatima Lakha }}{ }^{1}$, P Pennefather, P Ballantyne, A Mailis

${ }^{1}$ University of Toronto, Toronto, Ontario

POSTER AIM: Chronic non-cancer pain is a significant health problem in Canada. Little organized information exists on services currently available for the treatment of chronic non-cancer pain (CNCP) across Ontario. The aim of the study is to document teaching hospital-based pain clinic structures and services offered for management of CNCP in Toronto.

METHODS: Semi-structured, personal interviews with 4 key informants (clinic directors or one of his/her close collaborators), in charge of CNCP service delivery in 4 teaching hospitals were conducted. Each interview asked broad questions concerning infrastructure, research and professional development education. Strengths and barriers associated with each program were identified. Key informant interviews were audio taped, transcribed, and imported into spread-sheets for thematic analysis.

RESULTS: Preliminary analysis revealed that all clinics provided some form of multidisciplinary services by having a core team comprised of a pain physician, a nurse, and a psychologist/or physical therapist at minimum. The time between an accepted referral and consultation was approximately 2-6 months. If offered treatments are unsuccessful patients are discharged into community care, with no further follow-up. Common barriers for CNCP management include lack of resources (i.e. staff, space and funding), and need for systematic training, collaboration and research. CONCLUSIONS: The present study, first to our knowledge, demonstrated that multiple factors compromise the ability to provide comprehensive pain services even in academic pain clinics in Toronto. The study findings are important for administrators and policy makers interested in addressing the serious problem of chronic pain in Toronto and Ontario in general.

\section{P41}

UNDERSTANDING THE USE OF OVER-THE-COUNTER PAIN TREATMENTS IN ADOLESCENTS WITH CHRONIC PAIN: PERSPECTIVES OF ADOLESCENTS WITH CHRONIC PAIN AND THEIR PRIMARY CAREGIVERS

Lennifer Stinson ${ }^{1}$, F Campbell, L Isaac, S Brown, J Tyrrell, D Ruskin, M Jeavons, A Ayling Campos, C Lalloo, L Harris

${ }^{1}$ The Hospital for Sick Children, Toronto, Ontario

POSTER AIM: Chronic pain in youth aged 12 to 18 can negatively impact all aspects of health-related quality of life, as well as interfere with normative developmental activities. Youth with chronic pain predominantly manage pain in consultation with their primary care physician, while some are referred to specialized multidisciplinary pain clinics. These patients often use over-the-counter (OTC) oral and topical medications to manage pain while they seek specialized treatment. The aim of this investigation was to explore the use, decision-making process and communication about the use of OTC pain medications with health care professionals, in adolescents living with chronic pain and their primary caregiver. METHODS: A prospective, qualitative descriptive design with semistructured, audio taped individual interviews was undertaken with youth with chronic pain and their caregivers. A convenience sample of patientcaregiver dyads was recruited from SickKids chronic pain clinic.

RESULTS: Interviews were completed with children and adolescents $(n=6$, aged 13-18), and their caregivers $(n=7)$. Five themes were explored: (1) Prevalence of OTC medications; (2) Experiences using oral OTC medications; (3) Experiences using topical OTC medications; (4)
Perceived trustworthiness of OTC medications; and (5) Decision-making for use of OTC medications. The most significant decision-making factors were: recommendation from a trusted source (health care provider or personal contact), and potential drug-drug interactions. Healthcare providers had inconsistent involvement in the decision-making process.

CONCLUSIONS: Improved understanding of prevalence of use, the decision making process around use, and how patients and their families communicate about the use of OTC with healthcare providers can help clinicians better personalize treatments and help youth make sound self-care decisions. Next steps include further interviews until saturation is achieved, and future studies to understand the healthcare providers' experiences with OTC. This study can inform the development of better patient education and communication strategies about OTC pain medications.

\section{P42}

VALIDATION OF THE CRITICAL-CARE PAIN OBSERVATION TOOL AND VITAL SIGNS IN RELATION WITH THE SENSORY AND AFFECTIVE COMPONENTS OF PAIN DURING MEDIASTINAL TUBE REMOVAL IN POSTOPERATIVE CARDIAC SURGERY INTENSIVE CARE UNIT ADULTS

\section{Madalina Boitor ${ }^{1}$, JL Fiola, C Gelinas}

${ }^{1}$ McGill University, Montreal, Quebec

POSTER AIM: This study aimed to validate the use of the Critical-Care Pain Observation Tool (CPOT) and vital signs in association with the sensory and affective components of pain during mediastinal tube removal in the postoperative cardiac surgery intensive care unit (ICU) adults.

METHODS: A sample of 125 patients participated in this prospective repeated-measure within-subject study. A total of six assessments of the main study variables (CPOT scores and patients' self-reports of pain intensity and unpleasantness) were completed by trained research assistants while patients were in the ICU. Assessments were done before, during and 15 minutes after the non-nociceptive procedure (i.e. NIBP - non-invasive blood pressure taking) and nociceptive procedure (i.e. MTR - mediastinal tube removal). RESULTS: Discriminant validation of the scale use was supported as higher CPOT scores were obtained during MTR (mean=2.74, SD=1.61) compared to NIBP (mean=0.50, SD=0.747) $(\mathrm{t} 124=14.33, \mathrm{p}<0.001)$. Similarly, higher values were recorded for mean arterial pressure, heart and respiratory rates during MTR compared to NIBP $(\mathrm{p}<0.01)$. During MTR, CPOT scores correlated significantly with self-report of pain intensity $(\mathrm{r}=0.419, \mathrm{p}<0.01)$ and unpleasantness $(\mathrm{r}=0.313, \mathrm{p}<0.01)$ supporting criterion and convergent validation. Conversely, vital signs did not correlate with either self-report.

CONCLUSIONS: Study findings confirmed the validity of the CPOT for the assessment of the sensory and affective components of pain in the postcardiac surgery ICU adults. Vital signs were not specific to pain, and should only be used as cues to begin further assessment of pain using validated tools for this purpose.

\section{P43}

PATIENT MEDICATION USAGE PRE AND POST ADMISSION TO A MULTIDISCIPLINARY INPATIENT
COMPLEX PAIN PROGRAM

Ingrid Fedoroff ${ }^{1}$, M Ong-Lam, A Borowska

${ }^{1}$ St Paul's Hospital, Providence Health Care, Vancouver, British Columbia

POSTER AIM: Chronic pain is a complex interaction of physiological, psychological and psychosocial factors that impact all aspects of functioning in an individual (Turk \& Okifuji, 2002). The 'gold standard' for treatment is multidisciplinary and is often conducted via outpatient services. There are few programs that offer multidisciplinary treatment in an inpatient setting. The aim of this current study is to examine the efficacy of a biopsychosocial model of an interdisciplinary chronic pain inpatient program with respect to medication utilization. Multidisciplinary treatment included medicine, psychiatry, physiotherapy, occupational therapy, and psychology.

METHODS: A retrospective chart review (2 years) of patients admitted to an inpatient complex pain program was conducted.

RESULTS: During this time there were 96 admissions of which 59 (61\%) 
patients were taking opioids. Upon discharge 8/59 (13.5\%) were no longer on opioids. There was an average $11 \%$ reduction in overall opioid dosages (morphine equivalent doses were calculated) upon discharge. Analyses of patterns of types of opioids indicate a shift away from utilizing opioids with a higher abuse potential to ones with a lower abuse potential (i.e., methadone, codeine), greater utilization of longer acting versus shorter acting opioids at discharge and more use of adjuvant (nonopioid) medications. The complex nature of these patients is profiled with respect to pain and psychiatric diagnoses.

CONCLUSIONS: Inpatient treatment can offer a unique environment to make significant medication changes and offer alternative multimodal medical interventions to help reduce pain and hence patient analgesic needs.

\section{P44}

\section{EXPLORING THE RELATIONSHIP BETWEEN SELF-} COMPASSION, CHRONIC PAIN AND WELL-BEING

\section{Mary McCarthy ${ }^{1}$, D Cane}

${ }^{1}$ Capital Health Pain Management Unit, Halifax, Nova Scotia

POSTER AIM: Self-compassion (recognizing and accepting when one is experiencing difficulties and responding to oneself with kindness) may be a significant factor in the relationship between chronic pain and physical and psychological well-being. Higher levels of self-compassion have been found to be related to greater pain acceptance, more positive affect, reduced catastrophizing, and less disability. The present study examined the relationship between self-compassion and pain, pain control, affect, catastrophizing, and pain-related disability, and examined the impact of a pain self-management program on self-compassion. Gender differences in self-compassion were also examined.

METHODS: Sixty-eight individuals with chronic pain attending a pain self-management program completed questionnaires that included the Self-Compassion Scale, the PROMIS-29, the Survey of Pain Attitudes, and the Pain Catastrophizing Scale. A subset of patients ( $n=35)$ completed the same measures four weeks later at the conclusion of treatment.

RESULTS: Results indicated no significant differences between men and women with respect to self-compassion at both the beginning and end of treatment. No change in self-compassion was observed for men completing treatment but a trend toward increased self-compassion was noted for women. At the start of treatment greater self-compassion was associated with less pain, better pain control, less depressive symptomatology, and less pain catastrophizing. Self-compassion was not related to anxiety or painrelated disability.

CONCLUSIONS: The results suggest a relationship between self-compassion pain intensity, pain control, and psychological functioning among individuals with chronic pain. The results also suggest that attending a pain self-management program may be associated with increased self-compassion.

\section{P45}

\section{SELF-COMPASSION: A POTENTIAL FACILITATOR OF} ACTIVITY PACING?

\section{Douglas Cane ${ }^{1}$, M McCarthy}

\section{${ }^{1}$ Capital Health Pain Management Unit, Halifax, Nova Scotia}

POSTER AIM: Although instruction in pacing is typically included in the treatment of chronic pain, studies suggest that individuals encounter difficulty employing pacing. Pacing may require individuals to adopt an approach to activities that is perceived as less satisfactory than previous approaches. Self-compassion, which includes accepting personal shortcomings and difficulties without critical judgment, may be an important quality that enables individuals to better tolerate pacing and use it more frequently. The present study examined the relationships between self-compassion, perceived barriers to pacing, and pacing among individuals with chronic pain.

METHODS: Sixty-eight individuals with chronic pain attending a pain self-management program completed measures of self-compassion (SelfCompassion Scale), perceived obstacles to pacing (Pacing Obstacles Questionnaire), and pacing (Patterns of Activity - Pain Pacing Scale) prior to treatment.

RESULTS: Results indicated that self-compassion was significantly related to both perceived obstacles to pacing and pacing. Individuals who evidenced greater self-compassion identified fewer obstacles to pacing and a greater use of pacing. Regression analyses indicated that self-compassion and perceived obstacles to pacing both contributed independently to the prediction of pacing. Individuals who were less likely to negatively judge themselves for their perceived shortcomings and less likely to perceive shortcomings as unique to them identified fewer barriers to pacing.

CONCLUSIONS: The present results suggest that increased self-compassion may assist individuals to better accept the challenges associated with pacing and more frequently employ it as a pain management strategy. Specifically, a willingness to accept personal limitations without self-criticism and to view these limitations as being shared with others may facilitate the use of pacing.

\section{P46}

DISTRACTION FROM PAIN BY A WORKING MEMORY TASK - EVIDENCE FOR A TRADEOFF BETWEEN PAIN AND TASK PROCESSING

Vanessa Tabry ${ }^{1}$, P Brouillard, M Lussier, J Buhle, P Rainville,

L Bherer, M Roy

${ }^{1}$ Concordia University, Montreal, Quebec

POSTER AIM: Pain disrupts activities to call attention to a source of potential injury. This effect can become problematic, such as in chronic pain, which is nearly systematically associated with executive dysfunctions. Conversely, a recent study has found distraction by a demanding task reduces the intensity of a simultaneous painful stimulus, suggesting a tradeoff between pain and cognitive processing. We propose to examine the tradeoff between task performance and perception of concurrently received pain stimuli, and to further verify whether this tradeoff is correlated with executive functions ability, responsible for optimal cognitive resource allocation. METHODS: 20 young adult participants completed a challenging working memory task while receiving painful thermal stimuli, the parameters of which are individually calibrated for task difficulty and pain intensity beforehand.

RESULTS: Absorption in the challenging task significantly inhibits pain $(t(19)=2.5876, p=0.018)$ compared to the nonexecutive task, and pain has a significant effect on cognitive task performance $(t(19)=2.367, p=0.029)$, compared to warmth. Better task performance is correlated with lower pain on a trial-by-trial basis $(r=-0.223, \mathrm{p}=0.003)$.

CONCLUSIONS: There is evidence for a disruptive effect of pain on task performance and of an analgesic effect of task absorption on pain. The interindividual variability in this tradeoff and its neuropsychological and psychological predictors will be discussed in future analyses.

\section{P47}

\section{FAMILY PHYSICIANS AND TREATMENT OF CHRONIC NON-CANCER PAIN: AN EDUCATIONAL NEEDS ASSESSMENT}

Nancy Julien ${ }^{1}$, H St-Amant, M Ware, A Lacasse

${ }^{1}$ Université du Québec en Abitibi-Témiscamingue, Quebec

POSTER AIM: We aimed to assess educational needs of family physicians practicing in a northern region of the province of Quebec concerning chronic non-cancer pain (CNCP) and its treatment.

METHODS: In 2013, a postal survey was sent to all family physicians $(\mathrm{n}=183)$ in the region of Abitibi-Témiscamingue (Quebec) using an adapted Dillman Total Design Method. Using a 6-point Likert scale, they were asked about their current and desired level of knowledge about CNCP and its treatment, continuing medical educational (CME) activities attended in the past year, and perceived prevalence of CNCP among their clientele.

RESULTS: A total of 93 family physicians returned the questionnaire (response rate: $50.8 \%$ ). Survey respondents estimated the prevalence of CNCP among their clientele to be $25.9 \%( \pm 16.8 \%)$. Among respondents, $87.9 \%$ attended $\mathrm{CME}$ activities about $\mathrm{CNCP}$ and its treatment during the past year. However, $57.1 \%$ estimated that $\mathrm{CNCP}$ and its treatment represented only $1-10 \%$ of the content of those CME activities. The mean current knowledge level about $\mathrm{CNCP}$ and its treatment was 3.1/5 \pm 1.1 , while the mean desired knowledge was $4.4 / 5 \pm 0.9$. Participating physicians thus desired an average $28 \%(1.4 / 5 \pm 0.9)$ increase in their knowledge. A total 
of $91 \%$ of participating physicians desired a $4 / 5$ or a $5 / 5$ knowledge level. CONCLUSIONS: Survey participants in the region of AbitibiTémiscamingue (Quebec) desire more knowledge about CNCP and its treatment. This could be attained by more CME activities.

\section{P48 \\ "METHADONE REGISTRY", A PROSPECTIVE AUDIT OF PATIENTS WITH CHRONIC NON-CANCEROUS PAIN TREATED WITH METHADONE AT THE ALAN EDWARDS PAIN MANAGEMENT UNIT (AEPMU), MCGILL UNIVERSITY HEALTH CENTRE \\ Jordi Perez ${ }^{1}$, Y Shir \\ ${ }^{1}$ McGill University Health Centre, Montreal, Quebec}

POSTER AIM: To prospectively follow chronic non cancer pain (CNCP) patients treated with methadone at the AEPMU for their demographic characteristics, indication for therapy, methadone dose and therapeutic outcome during a five year period.

METHODS: Patients already taking methadone or receiving it for first time will be recruited for this registry and followed during five years. Research variables: 1 . Demographic data (gender, age, working and disability status, substance consumption); 2. CNCP problem (Diagnosis, triggering event and onset and duration); 3. Other concurrent illnesses; 4. Previous analgesic therapies; 5. Indication for methadone and dosing (formulation, posology, total daily dose) (in methadone naïve patients) 6. Current drug pain therapy (opioid and non-opioids, adjuvants) 7. Current non-drug pain therapy (physical, psychological, invasive interventions, CAM) 8. Side effects profile with current therapy 9 . Satisfaction with methadone therapy (if taking) 10. Brief Pain Inventory short form (BPI sf) 11. Short Form Health Survey (SF-12).

RESULTS: During the first three months of the registry we have enrolled 30 patients. Overall the registry has been widely accepted among clinicians and patients. By the time of the CPS conference, authors will present a summary of ongoing results.

CONCLUSIONS: A methadone registry like this one will provide a better understanding of patients' profile and documentation of the outcome of methadone therapy.

REFERENCES

1. Davis MP. Methadone for relief of cancer pain: a review of pharmacokinetics, pharmacodynamics, drug interactions and protocols of administration. Support Care Cancer 2001.

2. Gardner-Nix Oral methadone for managing chronic nonmalignant pain. J Pain Symptom Manage 1996.

3. Peng P. Experience of methadone therapy in 100 consecutive chronic pain patients in a multidisciplinary pain center. Pain Med 2008.

\section{P49}

TETRODOTOXIN FOR MODERATE TO SEVERE CANCERRELATED PAIN: A MULTICENTRE, RANDOMIZED, DOUBLEBLIND, PLACEBO-CONTROLLED, PARALLEL-DESIGN TRIAL

Neil A Hagen ${ }^{1}$, L Cantin, J Constant, T Haller, G Blaise, M Ong-Lam, P du Souich, W Korz, B Lapointe

${ }^{1}$ University of Calgary, Calgary, Alberta

POSTER AIM: This study evaluated subcutaneous tetrodotoxin (TTX) for the treatment of moderate to severe, inadequately controlled cancerrelated pain.

METHODS: Eligible subjects were randomized to receive TTX (30 $\mu \mathrm{g})$ or placebo subcutaneously twice daily for four consecutive days. Efficacy was assessed using primary Pain and Composite (which included Pain and QoL measures) endpoints adjusted for multiplicity. Safety was evaluated using AEs, 12-lead ECG, neurological examinations, and routine clinical tests. RESULTS: 165 subjects enrolled at 19 sites in Canada, Australia and New Zealand, with 149 in the primary analysis. A responder for the pain endpoint was defined as a subject with $\geq 30 \%$ reduction of pain score compared to baseline. Unadjusted responder rate analysis supports a clinical benefit on the primary pain endpoint, significant at the one-sided $5 \%$ level $(\mathrm{p}=0.0460)$ but not at the pre-specified two-sided $5 \%$ level. After accounting for age, daily opioid dose and pain level, the primary pain endpoint produced an estimated responder rate difference of $23.1 \%$, nominal $\mathrm{p}=0.0127$ and $\mathrm{NNT}=4.3$. The median duration of pain response was 12 days with TTX compared to 8 days for placebo $(\mathrm{p}=0.0345)$. The Patient Global Impression of Change supported an analgesic benefit $(\mathrm{p} \leq 0.0003)$. Most common AEs were nausea, dizziness, and oral hypoaesthesia or paresthesia. The majority of AEs reported were mild to moderate, transient, self-limiting, and could be managed with standard supportive care.

CONCLUSIONS: TTX can provide clinically meaningful cancer pain management for moderate to severe cancer pain in addition to opioid therapy or in opioid intolerant patients with a favorable benefit risk profile.

\section{P50}

AN EXAMINATION OF THE ASSOCIATION BETWEEN PAIN AND FAMILY FUNCTIONING USING THE INTERRAI CHILD AND YOUTH MENTAL HEALTH (CHYMH) INSTRUMENT

\section{Natasha Gallant ${ }^{1}$, K Arbeau, C Liu, S Stewart}

${ }^{1}$ University of Regina, Regina, Saskatchewan

POSTER AIM: Research has demonstrated an association between pain and parental/family variables in children and youth (e.g., Palermo \& Chambers, 2005). The present study investigated the influence of parental/ family variables on pain in samples of children and youth receiving mental health services.

METHODS: In this study, $\mathrm{n}=461$ children and youth $4-18$ years old $(\mathrm{M}=11.1, \mathrm{SD}=3.4)$ admitted to eight mental health agencies in Ontario, Canada were examined. Data was collected using the interRAI Child and Youth Mental Health (ChYMH) instrument (Stewart et al., 2014). The following variables were included: pain intensity, family functioning, caregiver wellbeing, and parenting strengths. Analyses were conducted using Kruskal-Wallis and Mann-Whitney U tests.

RESULTS: Results demonstrated that children and youth who experienced higher levels of pain intensity had worse family functioning, caregiver wellbeing, and parenting strengths when compared to those experiencing no pain.

CONCLUSIONS: These results illustrate the importance of examining parental/family indicators when developing pain management interventions for children and youth.

\section{REFERENCES}

1. Palermo TM, Chambers CT. (2005). Parent and family factors in pediatric chronic pain and disability: An integrative approach. Pain, 119, 1-4.

2. Stewart SL, Hirdes JP, Curtin-Telegdi N, et al. (2014). interRAI Child and Youth Mental Health (ChYMH) Assessment Form and User's Manual. Version 1. Washington, DC: interRAI.

\section{P51}

ARE WE MAKING THE GRADE? AN ASSESSMENT OF THE QUALITY OF THE EVIDENCE FOR SKIN-TO-SKIN CONTACT IN REDUCING NEWBORN PAIN

\section{Timothy Disher ${ }^{1}$, B Benoit, C Johnston, M Campbell-Yeo}

${ }^{1}$ IWK Health Centre, Halifax, Nova Scotia

POSTER AIM: To assess the level of evidence for the use of skin-to-skin care (SSC) provided by mothers to reduce procedural pain in preterm infants when compared to standard care.

METHODS: GRADE assessment of the findings of a Cochrane systematic review published by the co-authors. Outcomes include premature infant pain profile (PIPP), and heart rate (HR) during and after the procedure. RESULTS: For PIPP, Level of evidence ranged from moderate at 30 seconds, to very low at 120 seconds. Outcomes at each time point were downgraded as a result of inconsistency, where measures of heterogeneity ranged from $67-79 \%$. The 60,90 , and 120 second time points were additionally downgraded as estimated effect crossed the minimum important difference. HR during and after the procedure were both downgraded for risk of bias and imprecision. CONCLUSIONS: The quality of evidence for the effect of SSC on HR is low and provides no evidence of effect, although there was a promising trend towards lower HR after the procedure. While heterogeneity impairs our ability to confidently estimate the magnitude of effect of SSC care on PIPP scores, the quality of evidence was moderate at 30 seconds, and no studies 
reported any adverse events. Owing to the numerous benefits of skin-to-skin care for infants and mothers in non-pain contexts, we recommend its use as analgesia for a single painful procedure. Future research should seek to understand causes of heterogeneity in PIPP scores, with dose of KC, assessor skill levels, and potential bias from unblinding as the most likely sources.

\section{P52}

TRENDS IN TRAMADOL USE AND ABUSE REPORTED TO THE ONTARIO POISON CENTRE

Emily Austin ${ }^{1}$, M Thompson, H Hudson, D Bernabeo, DJG Johnson, R Dart

${ }^{1}$ Ontario Poison Centre, University of Toronto, Toronto, Ontario

POSTER AIM: Tramadol is a synthetic opioid marketed for treatment of moderate pain. Tramadol may have abuse potential. We describe the incidence of intentional tramadol exposures for which the Ontario Poison Centre (OPC) was consulted between 2011 and 2013.

METHODS: All intentional human exposures to tramadol-containing products for which the OPC was consulted in calendar years 2011 to 2013 were examined to identify trends. Intentional exposures included suspected suicide, misuse, abuse, and unknown. Drug data on numbers of tramadol tablets sold annually in Canada were included.

RESULTS: OPC calls increased yearly from 52,184 in 2011 to 58,712 in 2013. Calls for intentional tramadol exposures increased from 68 to 75 in that time but remained at a constant $0.13 \%$ of all human exposure calls. Suspected suicide was the predominant reason $(76 \%, 86 \%$ and $75 \%$ for 2011, 2012, and 2013 respectively). The percentage of calls relating to abuse and misuse varied. Tramadol exposures related to suspected suicide were also associated with more serious clinical effects (critical care admissions, death). Importantly, the number of prescriptions for tramadol increased yearly during this time, as reflected by an increase in the number of standard dose units sold per 100, 000 population by $12 \%$ in 2012, and 16\% in 2013 .

CONCLUSIONS: OPC experienced an increase in the number of calls relating to intentional tramadol exposures from 2011-2013. Given the increasing number of prescriptions sold, more data about its use and abuse should be monitored as it is not scheduled.

\section{P53}

SYSTEMATIC REVIEW OF CHILDHOOD AND ADOLESCENT RISK AND PROGNOSTIC FACTORS FOR MUSCULOSKELETAL PAIN

Michelle E Tougas ${ }^{1}$, A Huguet, J Hayden, PJ McGrath, C Chambers, JN Stinson

${ }^{1}$ IWK Health Centre, Dalhousie University, Halifax, Nova Scotia

POSTER AIM: Little is known about musculoskeletal pain risk and prognostic factors. This systematic review examined evidence about childhood and adolescent factors associated with onset and persistence of musculoskeletal pain.

METHODS: Electronic databases, references of included studies, and the Pediatric Pain mail list were searched. Data was extracted about study and patient characteristics, prognostic factors, outcome variables, data analysis, and results. The quality of this evidence was evaluated using a modified GRADE framework.

RESULTS: Two reviewers screened 2010 full text articles, including 31 studies reporting 18 cohorts. From the included studies 61 risk factors for onset and 43 prognostic factors for persistence of musculoskeletal pain were explored. Factors were grouped according to the International Classification of Functioning, Disability and Health for Children and Youth. The quality of evidence is low or very low for prognostic factors associated with persistence of musculoskeletal pain. Moderate quality evidence suggests the childhood factors poor regulation of emotions, high body mass and smoking are not significantly associated with risk of musculoskeletal pain onset. Moderate quality evidence from two studies suggests that children and adolescents with headache or abdominal pain may be at increased risk for onset of musculoskeletal pain. Moderate quality evidence suggests that high levels of weekly physical activity predicted onset of low back pain.

CONCLUSIONS: Due to the small number of studies, poor quality of evidence, and heterogeneity of the research, additional well-conducted primary studies are needed to increase confidence in evidence and to explore new childhood risk and prognostic factors for musculoskeletal pain.

\section{P54}

\section{ANXIETY AND PAIN CATASTROPHIZING AS PREDICTORS FOR DULOXETINE EFFICACY IN THE TREATMENT OF FIBROMYALGIA}

Lydia Girard-Tremblay ${ }^{1}$, K Daigle, A Masetto, G Boire, S Marchand, P Goffaux

${ }^{1}$ Université de Sherbrooke, Sherbrooke, Quebec

POSTER AIM: Duloxetine is a SNRI used in the management of fibromyalgia (FM). Although some patients clearly benefit from treatment with duloxetine, many do not experience satisfying symptom relief, and it is not clear why this response difference exists. The aim of this study was to investigate if some individual characteristics could predict response to treatment in FM patients. METHODS: 14 patients with FM participated in this study. Treatment response was assessed by comparing FM symptoms prior to and after a 4-week treatment with duloxetine, using the Brief Pain Inventory, Fibromyalgia Impact Questionnaire, State-Trait Anxiety Inventory and Pain Catastrophizing Scale. Associations between variables were determined using Pearson correlation analysis.

RESULTS: Lower pain catastrophizing states (mainly rumination and helplessness) prior to treatment were associated with greater treatment-induced improvements in pain severity, pain-induced interference and impact of pain on everyday life, as well as higher subjective impressions of change on global health status (GHS). Lower anxiety scores prior to treatment were also associated with a greater reduction of pain severity and lesser negative sideeffects of treatment on sleep quality. Finally, older age was associated with greater treatment-induced reductions in pain severity, greater improvements in pain interference, and higher impressions of change on GHS.

CONCLUSIONS: Patients with lower anxiety and pain catastrophizing scores before treatment were more likely to experience duloxetine as effective. This finding further strengthens the idea that psycho-biological processes are important to consider when seeking to maximise treatment efficacy. Here we show that a less anxious, less catastrophizing mind will benefit most from pharmacological treatment.

\section{P55}

\section{EVOLUTION OF DISABILITY IN WORKERS WITH LOW BACK PAIN IS ASSOCIATED WITH PSYCHOLOGICAL SYMPTOMS}

Jean-Daniel Dubois ${ }^{1}, M$ Piché, M Descarreaux

${ }^{1}$ Université du Québec à Trois-Rivières, Trois-Rivières, Quebec

POSTER AIM: The pathophysiology underlying chronic low back pain (LBP) is largely unknown, yet growing evidence suggests that complex interactions between individuals and the environment are likely responsible for increased susceptibility to chronic pain conditions. As such, numerous factors such as increased psychological symptoms, altered trunk muscle activation, pain thresholds and pain modulation mechanisms have been explored in cross-sectional studies as possible determinants of disability in individuals with LBP. The aim of this study was to determine if these factors measured initially could be associated with disability six months after the initial assessment.

METHODS: Seventy-one workers with past history of LBP were recruited for a longitudinal study including three sessions (initial, three and six months). Experimental sessions (initial and six months) included assessment of thermal pain and tolerance thresholds, pain inhibition and trunk muscle activation during a dynamic task with and without cutaneous heat pain applied to the low back. All sessions included validated questionnaires to examine pain catastrophizing, fear-avoidance beliefs and pain hypervigilance. Clinical pain and disability were also assessed. Principal components were created for psychological questionnaires, pain thresholds and tolerance thresholds as well as for EMG responses. Correlation analyses were conducted to determine the association between these components, and disability levels at six months.

RESULTS: Disability at six months was only associated with the psychological questionnaires component $(\mathrm{r}=0.461, \mathrm{p}<0.001)$. 
CONCLUSIONS: The complex nature of LBP makes it difficult to determine its evolution. Nevertheless, increased psychological symptoms and negative affect are associated with increased disability at 6 months.

\section{P56}

SEX DIFFERENCES IN NEONATAL PAIN RESPONSE: A NARRATIVE SYNTHESIS AND ANALYSIS OF THE CURRENT STATE OF KNOWLEDGE

Britney L Benoit ${ }^{1}$, T Disher, M Campbell-Yeo

${ }^{1}$ Dalhousie University School of Nursing, Halifax, Nova Scotia

POSTER AIM: Neonatal pain assessment and management practices do not consider sex-specific differences in pain response. The aim of this work was to synthesize the current state of knowledge concerning sex-based differences in infant pain response to inform recommendations for future research and practice.

METHODS: A search of key electronic databases (CINAHL, PubMed, PsychINFO) was conducted in November 2014. Key words were: Sex, sex difference, pain, procedure, heel stick or lance, venipuncture, arterial line, nasogastric tube insertion, response, reaction, behavioral response, infant, neonate, preterm, low or very low birth weight. All abstracts were reviewed and 13 English articles that reported differences in acute pain responses for male and female infants were retained.

RESULTS: There was significant variability across different painful procedures (heel lance, venipuncture, intramuscular injection), assessment measures (behavioral, physiologic, cortical), and gestational ages (24-42 weeks), with 7 studies reporting sex-based differences in pain response. Of the $13(\mathrm{n}=823)$ studies, two examined sex differences as the primary outcome and two controlled for baseline differences in factors known to influence pain reactivity (e.g., gestational age, previous painful procedures) between male and female infants. Across gestational ages, a trend existed in infant girls demonstrating increased behavioral responses and infant boys demonstrating more robust physiologic responses.

CONCLUSIONS: Limited evidence suggests that there may be sex differences in neonatal pain response. There is a need for further research aiming to identify potential differences in immediate response, response to interventions, and later development following pain exposure to inform pain assessment and management practices in infants.

\section{P57}

\section{SPINAL CORD STIMULATION ALTERS CORTICAL PAIN} EVOKED RESPONSES IN NEUROPATHIC PAIN PATIENTS

\section{Cecile de Vos ${ }^{1}$, JG Niso Galan}

\section{${ }^{1}$ Medisch Spectrum Twente, Enschede, Netherlands}

POSTER AIM: Spinal cord stimulation (SCS) provides significant pain relief in $65 \%$ of patients with otherwise intractable neuropathic pain. However, its working mechanisms remain largely unknown and it is therefore also unknown why little to no pain relief is obtained in 35\% of the patients. This pilot study investigates whether SCS modulates cortical processing of painful stimuli in order to better understand SCS working mechanisms.

METHODS: Patients with unilateral neuropathic pain and effective SCS were recruited. Intra-epidermal electrical stimulation (IES) was applied via a needle electrode designed to stimulate Adelta fibres exclusively (1). Thirty electrical pulses (duration: $500 \mu$ duration, amplitude: 4 times sensation threshold) were applied to the painful and the homologous nonpainful body sides, with SCS switched ON and OFF. The inter-stimulus interval was varied between 3.5-10 s. 64-channel EEG (sampling rate $5 \mathrm{kHz}$ ) was recorded continuously during stimulation. Data processing and calculation of the evoked potentials (EP) were performed post hoc using Brainstorm Software (2).

RESULTS: So far, three patients participated and all sensed the IES in all conditions. In general, IES applied to the non-painful side evoked clear vertex EPs and IES applied to the painful side evoked attenuated EPs. The amplitude of the vertex EP was increased when SCS was switched ON compared to when SCS was switched OFF, irrespectively of the side stimulated. CONCLUSIONS: SCS modulates cortical processing of peripherally applied painful stimuli indicating that SCS leads to altered electrophysiological activity at both spinal and supraspinal levels.

\section{REFERENCES}

1. Inui K, Tran TD, Hoshiyama M, Kakigi R. Preferential stimulation of Adelta fibers by intra-epidermal needle electrode in humans. Pain 2002;96:247-52

2. Tadel F, Baillet S, Mosher JC, Pantazis D, Leahy RM. Brainstorm: A user-friendly application for MEG/EEG analysis. Computational intelligence and neuroscience, 2011;8. Available from: http://neuroimage.usc.edu/brainstorm

\section{P58}

\section{TRANSBORDER TRAFFICKING OF PRESCRIPTION OPIOIDS IN CANADA AND THE UNITED STATES}

\section{Stevan G Severtson ${ }^{1}$, JL Green, N Dasgupta, MS Ellis, TJ Cicero,} RC Dart

${ }^{1}$ Rocky Mountain Poison and Drug Center, Denver Health and Hospital Authority, Denver, Colorado, USA

POSTER AIM: This study uses surveillance data to examine cross-border trafficking of prescription opioids manufactured in Canada and the United States (US).

METHODS: Reports from two surveillance programs were analyzed: the StreetRx Program, a website that uses crowdsourcing to assess the street price of prescription and illicit drugs, and the Researchers and Participants Interacting Directly (RAPID) Program, a survey administered to a sample of treatment-seeking individuals in the US who are dependent on opioids. Cross-border trafficking into Canada was assessed using reports of oxymorphone, which is manufactured in the US and not available in Canada. Cross-border trafficking into the US was assessed using reports of extended release (ER) oxycodone products manufactured in Canada.

RESULTS: In US StreetRx, there were 169 reports of Canadian manufactured crushable ER oxycodone products between June 2012 and October 2014. These accounted for 7\% of ER oxycodone reports in the US. The median price per milligram was $\$ 1.00$. There were 4 OxyNEO reports with a median price per milligram of $\$ 0.60$. In Canadian StreetRx there were two reports of oxymorphone during the same time period. In RAPID, the most frequently reported product from Canada was OxyContin/OxyNEO, with 22 of 256 respondents (9\%) obtaining or using.

CONCLUSIONS: Illicit trade of prescription opioids across the border between Canada and the US does exist. Further research is needed to examine the extent to which this illicit trade persists.

\section{P59}

\section{CAREGIVER CULTURE, CAREGIVER BEHAVIOURS, AND INFANT IMMUNIZATION PAIN AT 2 MINUTES POST- NEEDLE AT 12 MONTHS OF AGE}

\section{Monica C O'Neill ${ }^{1}$, R Pillai Riddell, H Garfield, S Greenberg}

${ }^{1}$ York University, Toronto, Ontario

POSTER AIM: To examine how caregiver behaviors mediate the relationship between caregiver heritage culture and infant pain 2 minutes post-needle.

METHODS: A subsample of infants $(\mathrm{N}=364)$ with immunization data at 12 months of age was selected from a longitudinal cohort following healthy caregiver-infant dyads during routine immunizations over the first year of life. Infant facial expressions of pain were coded at 2 minutes post-needle (NFCS; Grunau \& Craig, 1987). Caregiver behaviours were coded for emotional availability (EAS; Biringen, 2008) and proximal soothing (MAISD; Cohen et al., 2005). Heritage culture was operationalized by a novel index created from an objectively derived 'individualism' rating assigned to the caregiver's self-identified heritage culture and the caregiver's strength of identification with their self-identified heritage culture (i.e., the Heritage Culture Identification and Individualism Index [HCIII]).

RESULTS: A mediation analysis revealed there was a significant indirect effect of HCIII scores on infant pain at 2 minutes, through caregiver emotional availability $(\mathrm{B}=-0.0017 ; 95 \%[-0.0045,-0.0006])$, but not through proximal soothing $(B=0.0005 ; 95 \%[-0.0001,0.0017])$.

CONCLUSIONS: Higher HCIII scores (i.e., meaning caregivers had a high identification with a heritage culture that was high on individualism) 
predicted greater emotional availability, which in turn predicted lower infant pain expression at 2 minutes post-needle. The indirect effect of HCIII scores on infant pain through caregiver proximal soothing was nonsignificant. The present findings further our understanding of the mechanism by which caregiver culture (and identification with that culture) impacts infant acute pain expression.

\section{P60}

INTERDISCIPLINARY MANAGEMENT OF SUBACUTE LOW BACK PAIN IN PRIMARY CARE: PRELIMINARY EVALUATION OF A NOVEL PROGRAM IN QUEBEC

\section{Mark Ware ${ }^{1}$, S Ahmed, O Eilayyan, R Visca}

\section{${ }^{1}$ McGill University, Montreal, Quebec}

POSTER AIM: Patients who do not respond to conventional back pain management algorithms six weeks after the onset of acute pain present an opportunity to identify barriers and implement treatment aimed at preventing the development of chronic low back pain. We present a preliminary evaluation of a novel interdisciplinary approach to subacute low back pain (pain between 6 weeks and 12 months of onset) implemented in a primary care setting.

METHODS: A six month interdisciplinary (physician, psychologist, physiotherapist and nurse) program was developed based on chronic care models (Wagner), self-management principles and best practice criteria for diagnosing and treating mechanical low back pain. The program has been implemented in four primary care centres across Quebec. Evaluation was based on pain, disability and quality of life measures using the Brief Pain Inventory (BPI), Oswestry Disability Index (ODI) and SF12v2 respectively, administered at baseline, one, three and six month intervals.

RESULTS: Up to 30 September 2014, 152 patients seen at the four sites (mean age 51.3y (SD 4.7); 71\% female). Mean baseline was moderate (VAS intensity 5.2 (SD 2.0; VAS interference 5.4 (SD 2.6)). Moderate to severe risk of chronicity (StarT BACK scale) was present in $75 \%$ of patients. Preliminary analysis suggests improvement in pain intensity, interference, disability and mood, and qualitative analysis suggests satisfaction from patients and caregivers.

CONCLUSIONS: The program is ongoing and these preliminary data suggest that improvements in a broad range of outcomes are being observed. Ongoing program evaluation with respect to practitioner and patient satisfaction will help inform program development and wider implementation.

\section{P61}

\section{CLINICIANS' KNOWLEDGE OF THE CURRENT DIAGNOSTIC CRITERIA FOR MYOFASCIAL PAIN SYNDROME}

Liza Grosman-Rimon ${ }^{1}$, J Katz, H Clarke, S Ahmed, D Kumbhare

${ }^{1}$ Toronto Rehabilitation Institute, Toronto, Ontario

POSTER AIM: Myofascial pain syndrome (MPS) is the most common chronic pain disorder (prevalence: $\sim 15 \%$ in medical clinics to $\sim 85 \%$ in pain centres). However, even this common disorder is underdiagnosed and therefore undertreated. Students in Canadian medical schools have a deficiency in pain education, suggesting they do not receive adequate training in evaluating musculoskeletal conditions (e.g., MPS). The aim of this study was to compare practicing clinicians' knowledge of current diagnostic criteria for MPS.

METHODS: 119 clinicians completed a 14-item survey of current diagnostic criteria for MPS; including 7 correct and 7 incorrect statements. Items were rated on a Likert scale (1 (absolutely agree) to 7 (absolutely disagree)). Non-parametric test (Bonferroni-corrected) were used to compare 6 clinician groups (family physicians- $(n=48)$, physical medicine/rehab specialists- $(n=37)$, rheumatologists- $(n=13)$, emergency room $(E R)$ physicians- $(n=12)$, and anesthesiologists specializing in chronic pain- $(n=9)$. A p value $\leq 0.01$ was considered statistically significant.

RESULTS: Clinician responses differed from the true responses for correct (true responses $=1$, corresponding to "absolutely agree") and incorrect items true responses $=1$, corresponding to "absolutely agree"). Median scores (interquartile range $[\mathrm{IQR}]$ ) for correct items were 3 (2-4); 3 (2-4);
$3(3-4) ; 4(3-5) ; 2$ (2-3) for family physicians, physical medicine/rehab specialists, rheumatologists, ER physicians, and anesthesiologists, respectively. Anesthesiologists scored better than the others. ER physicians scored worst. Median scores (IQR) for incorrect items were $4(3-5)$; 4 (4-6); 4 (3-5); 4 (3-5); 4 (3-6), respectively. Physical medicine/rehab specialists scored slightly than family physicians rheumatologists, ER physicians, but not anesthesiologists.

CONCLUSIONS: The clinicians showed a lack of knowledge of MPS diagnosis. Future studies should examine the benefit of incorporating adequate training into the medical school curriculum.

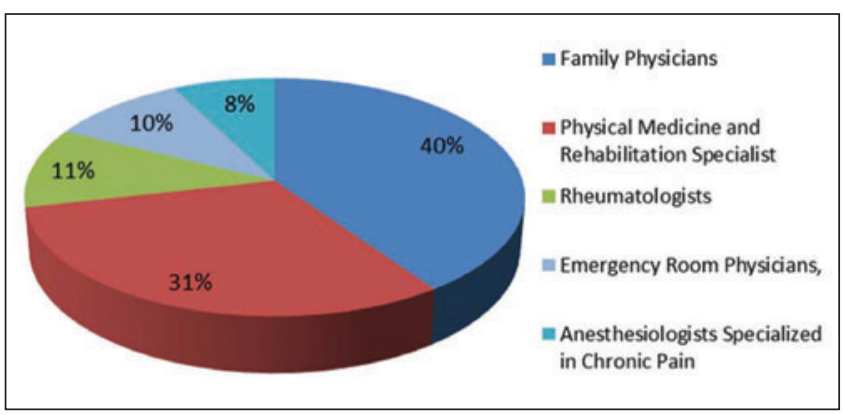

Figure 1) The percentages of each group of participants in the survey

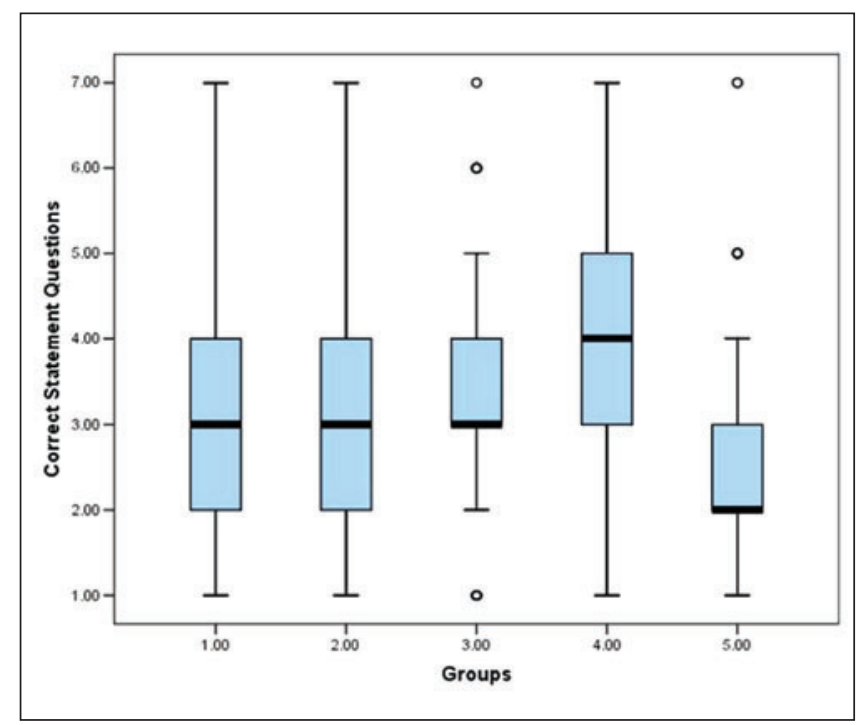

Figure 2A) Participants' median responses to the correct questions. The $x$ axis represents groups: family physicians (1), physical medicine and rehabilitation specialists (2), rheumatologists (3), emergency room physicians (4), and anesthesiologists specializing in chronic pain (5). The y axis represents responses: absolutely agree (1), strongly agree, (2) agree, (3) neutral (4), disagree (5) strongly disagree (6) absolutely disagree (7). The horizontal line in the centre of each box indicates the median. The top and the bottom borders of the box mark the 75th and 25th percentiles, respectively. Whiskers mark the 90 th and 10 th percentiles. A circle represents extreme outliers beyond the 90 th and 10 th percentiles. 


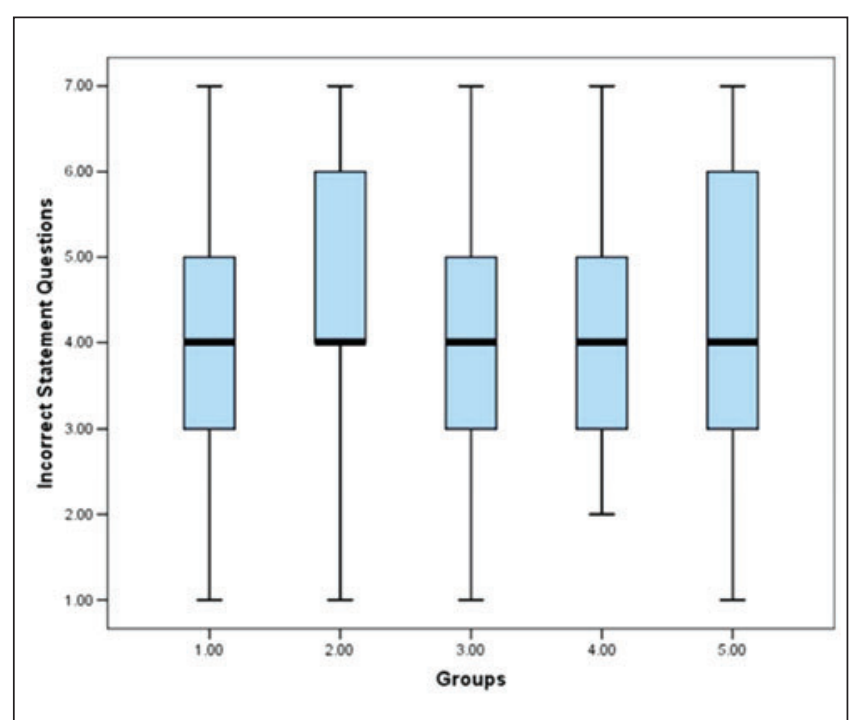

Figure 2B) Participants' medians responses to the incorrect questions. Family physicians (1), physical medicine and rehabilitation specialists (2), rheumatologists (3), emergency room physicians (4), and anesthesiologists specializing in chronic pain (5). The $x$ axis represents groups: family physicians (1), physical medicine and rehabilitation specialists (2), rheumatologists (3), emergency room physicians (4), and anesthesiologists specializing in chronic pain (5). The y axis represents responses: absolutely agree (1), strongly agree, (2) agree, (3) neutral (4), disagree (5) strongly disagree (6) absolutely disagree (7). The horizontal line in the centre of each box indicates the median. The top and the bottom borders of the box mark the 75th and 25th percentiles, respectively. Whiskers mark the 90 th and 10 th percentiles. A circle represents extreme outliers beyond the 90 th and 10th percentiles.

\section{P62}

\section{A PILOT STUDY: CIRCULATING BIOMARKERS IN MYOFASCIAL PAIN SYNDROME}

\section{Liza Grosman-Rimon'1, WL Parkinson, D Kumbhare}

${ }^{1}$ Toronto Rehabilitation Institute, Toronto, Ontario

POSTER AIM: An altered milieu of biomarkers has been reported to be associated with myofascial pain syndrome (MPS) with evidence of inflammation in the vicinity of active myofascial trigger points (MTrP). Importantly, patients with active MTrP, also have elevated levels of inflammatory biomarkers in remote uninvolved sites, suggesting a more generalized inflammation. We hypothesized that in patients with MPS circulating markers of inflammation will be elevated. The objective of our study was to assess the systemic levels of inflammatory markers and growth factors in patients with active MPS.

METHODS: The levels of systemic inflammatory markers macrophage inflammatory proteins (MIP-1a), macrophage-derived chemokine (MDC), granulocyte-macrophage colony-stimulating factor (GM-CSF), tumor necrosis factors-a (TNF-a), and growth factors fibroblast growth factor (FGF-2) and Fms-like tyrosine kinase 3 ligand (FLT-3L) were measured in 50 patients with MPS within the first week of symptoms and in 15 healthy controls, using Antibody-immobilized beads on a Luminex analyzer. Independent $\mathrm{t}$-tests were performed to examine the differences between the groups for each biomarker. Data are presented as mean \pm SEM. A p value $\mathrm{d} 0.01$ was considered statistically significant.

RESULTS: We found that the inflammatory markers MIP1-a $(22.53 \pm 3.57$ vs. $8.84 \pm 2.23, \mathrm{p}<0.01), \operatorname{MDC}(1411.85 \pm 76.95$ vs. $28.41 \pm 18.21, \mathrm{p}<0.01)$, GM-CSF $(88.03 \pm 14.20$ vs. $21.52 \pm 7.73, \mathrm{p}<0.01)$, TNF-a $(55.82 \pm 13.69$ vs. $11.56 \pm 5.16, \mathrm{p}<0.01)$ and growth factors FGF-2 $(237.91 \pm 19.27$ vs. $42.19 \pm 3.33, \mathrm{p}<0.01)$, FLT $-3 \mathrm{~L}(114.05 \pm 18.98$ vs. $14.11 \pm 3.20, \mathrm{p}<0.01)$ were elevated in patients with MPS compared to controls.

CONCLUSIONS: Future studies should address the mechanism for the increase in biomarkers. Investigating biomarker levels in MPS may be important for both diagnosis and treatment of MPS.

\section{P63}

BALANCING COMPLEXITY AND RISK: EXPERIENCES OF PAIN MANAGEMENT FOR MEN LIVING WITH HEMOPHILIA Susan Tupper ${ }^{1}$, PJ Downe, J Nilson, M Sims, K Brose, R Card, T Schlosser, N Hodgson 1Saskatoon Health Region, Saskatoon, Saskatchewan

POSTER AIM: Investigate the pain experience of adult men living with hemophilia, understanding of pharmaceutical treatment options, and information seeking about pain management.

METHODS: Two focus groups were conducted with 9 male participants enrolled through purposeful sampling of the Saskatchewan Bleeding Disorders Program. Focus groups followed brief education sessions on pharmaceutical pain management and medical marijuana. Participant ages ranged from 17 to 47 years. Data were analyzed using inductive thematic analysis by two independent researchers; composite categories and themes were developed.

RESULTS: Four primary themes were generated: pain experiences, risk, challenges, and information exchange. Pain is a common experience related to bleeds, persistent pain from arthropathies, and treatment (e.g. surgeries, factor injections). Men were concerned about side effects and toxicity of over-the-counter and prescribed medications. Even with a prescription, concern over negative social perceptions affected the use of marijuana. Negative physician attitudes led some participants to obtain marijuana for pain relief through illegal means. Challenges include limited knowledge of care providers, lack of pain management services, negative provider perceptions about drug seeking, and concerns of participants about treatment risks. Participants identified a need for education for the public and general practitioners about hemophilia and pain management, research on safe and effective pain management for people with hemophilia, and advocacy for better access to pain services. Information about pain management was primarily...

CONCLUSIONS: Pain is a complex co-morbidity associated with hemophilia that is a challenge for men and their care providers to manage effectively.

\section{P64}

THE RELATIONSHIP BETWEEN PREOPERATIVE PAIN-RELATED FEAR-AVOIDANCE AND POST-OPERATIVE PAIN AND PHYSICAL ACTIVITY AFTER PEDIATRIC MAJOR SURGERY

Brittany N Rosenbloom ${ }^{1}$, MG Page, F Campbell, L Isaac, J Stinson, S Razavi, K Chin, J Katz

${ }^{1}$ York University, Hospital for Sick Children, Toronto, Ontario

POSTER AIM: The development and maintenance of pediatric chronic post-surgical pain (CPSP) likely follows a Fear-Avoidance Model of chronic pain and disability. However, the fear/avoidance component of this model has not been comprehensively examined in children. The aim of this study was to evaluate the relationships among pediatric pain, pain-related fear/ avoidance, and physical activity in the days after major surgery.

METHODS: A prospective observational study was employed to follow 37 children $[24.3 \%$ male; mean age $=13.9(\mathrm{SD}=2.68)]$ perioperatively. Preoperatively, children completed questionnaires to examine pain [Numeric Rating Scale (NRS)], pain anxiety [Child Pain Anxiety Symptoms Scale (CPASS)], and fear of movement [Tampa Scale for Kinesiophobia (TSK)]. In-hospital activity was measured continuously for 3 days post-operatively using a non-invasive activity monitor (ActiCal).

RESULTS: Higher preoperative scores on both the CPASS $(\mathrm{r}(34)=$ $-0.35, \mathrm{p}=0.038)$ and TSK $(\mathrm{r}(34)=-0.37, \mathrm{p}=0.025)$ predicted significantly less activity on Day 1 (but not Days 2-3). Moreover, Day 1 movement-evoked pain (NRS-M) was significantly negatively correlated $(\mathrm{r}(34)=$ $-0.42, \mathrm{p}=0.009)$ with Day 1 activity (but not later). Interestingly, neither CPASS $(r(34)=0.20, p=0.698)$ nor TSK $(r(34)=-0.058, p=0.735)$ scores predicted mean NRS-M on Day 1 (or later).

CONCLUSIONS: The results demonstrate that higher levels of preoperative pain anxiety and fear of movement as well as movement-evoked pain on Day 1 after surgery was associated with less physical activity on Day 1. These results provide construct validity for the TSK in the pediatric post-surgical hospital setting. 


\section{P65}

A 2014 UPDATE OF THE PSYCHOMETRIC PROPERTIES, INTERPRETABILITY AND FEASIBILITY OF SELF-REPORT PAIN INTENSITY MEASURES FOR USE IN CLINICAL TRIALS IN CHILDREN AND ADOLESCENTS: A SYSTEMATIC LITERATURE REVIEW

Lennifer Stinson ${ }^{1}$, M Napoleone, C Nguyen, L Yohannes,

V Kazazian, B Chan, C Emanuele, S Luca, J Foster

${ }^{1}$ The Hospital for Sick Children, Toronto, Ontario

POSTER AIM: The aim of this study was to update the results of 2006 literature review that evaluated self-reported pain intensity measures for children and adolescents.

METHODS: Two separate searches were conducted in six databases for: (1) self-reported measures of single-item ratings of pain intensity and (2) Numerical Rating Scale measures, for children aged 3-18 years. Measures were excluded if they were disease specific and previously included in the 2006 review. Results of the search were divided among eight reviewers to screen for relevance. Two reviewers then assessed the potentially relevant studies for inclusion independently. Disagreements that arose were resolved by discussion including a third reviewer.

RESULTS: The overall database search resulted in 13,346 articles found (after duplicate removal). Screening for eligibility resulted in four new single-item measures to be included in the updated review: (1) Numerical Rating Scale, (2) Verbal Numerical Rating Scale, (3) Computer Face Scale and (4) Colour Analogue Scale. The four scales were determined to be psychometrically sound in terms of reliability, feasibility and responsiveness, but had differences in interpretability and feasibility. Similar to the previous review, no single scale was found to be optimal for use with all types of pain and across age groups.

CONCLUSIONS: This update in self-report pain intensity measures can be used to: (1) make recommendations on pain intensity measures to be used in the assessment of pediatric acute, recurrent, and chronic pain treatment strengthen and (2) guide future research in the use of pain scales for the pediatric population.

\section{P66}

\section{SUCCESSFUL DISSEMINATION OF THE STANFORD CHRONIC PAIN SELF-MANAGEMENT PROGRAM IN SOUTH-EASTERN ONTARIO}

Ruth E Dubin ${ }^{1}$, D Deptuck, A Young, C King Van-Vlack, M O'Leary, J Webb

\section{${ }^{1}$ ECHO Ontario}

POSTER AIM: In 2005, two of the authors (RED, CKVV) led the development of an exercise/education intervention for chronic pain (Y-PEP) which incorporated the Chronic Pain Self-Management Program (CPSMP) developed by Dr. Sandra Lefort (later licensed as the Stanford CPSMP). The program ran for 4 years in south-eastern Ontario (SELHIN), but despite our best efforts there was very limited expansion of the program. The Ministry of Health of Ontario started supporting Stanford selfmanagement programs in 2011, after which RED and CKVV completed the CPSMP Master trainer program. This led to networking opportunities in our region and rapid dissemination of the program.

RESULTS: Between 2006 and 2010, only three localities ran CPSMP's. By 2014, > fifteen sites provided CPSMP (some towns had multiple sites). By mid-2014 nearly 200 chronic pain sufferers had attended. Outcomes analysis (2012-2014) revealed that participants' confidence in performing regular activities, and in undertaking physical activity increased after attending the program. Effects were greater in those who had higher pain scores $(>5 / 10)$. The SELHIN now has 34 workshop leaders and 3 Master trainers.

CONCLUSIONS: Self-management is a core component of chronic pain management. The combination of advocacy, networking, and support from Ministry of Health - funded agencies achieved regional dissemination of the CPSMP, with improved functioning in attendees.

\section{P67}

PRODROMAL SYMPTOMS - A COMPLICATED CLINICAL CHALLENGE

Sheila O'Keefe-McCarthy, Linda Ready ${ }^{1}$

${ }^{1}$ City of Kawartha Lakes Family Health Team, Kawartha Lakes, Ontario

POSTER AIM: Prodromal symptoms (PS), indicative of myocardial ischemia are often not recognized as cardiac in nature. PS are transitory symptoms that may occur days to months prior to a myocardial infarction. The most prevalent PS reported by men and women included: unusual chest and arm pain, dyspnea, fatigue, dizziness, headache, increased sleep disturbance and anxiety. Purpose: To illustrate a typical diabetic follow up of a patient who presented with varied PS.

METHODS/RESULTS:

Assessment: A 65 year old female presented with a one month history of recurrent, unprovoked nausea and vomiting, intermittent chest discomfort and three week history of insomnia. Differential diagnoses included: cholecystitis, gastro-esophageal reflux or diabetic gastroparesis. Internal medicine consult suggested that symptoms were likely gastro-intestinal in nature and recommended an exercise stress test and echocardiogram.

Investigations: Echocardiogram revealed normal systolic function with mild diastolic dysfunction. Exercise stress test indicated moderate antero-septal and apical ischemia. Diagnostic cardiac catheterization revealed 99\% stenosis of the left anterior descending artery and $70 \%$ of the proximal right coronary artery.

Diagnosis: Obstructive coronary artery disease (CAD).

Treatment: A percutaneous coronary intervention with drug eluting stents was emergently performed.

CONCLUSIONS: PS, transient in nature, are cardinal signs or 'a red flag' to take note of that are reflective of suspicious myocardial ischemia. Mounting evidence documents that myocardial ischemia leading to infarction, specifically within the diabetic-cardiovascular population, may occur in the absence of pain. In fact, up to $30 \%$ of episodes of myocardial ischemia have been silent or painless, related to development of diabetic autonomic neuropathy. Routine pre-emptive screening of PS in clinical practice should be included and may serve to identify those patients at increased risk for CAD. Moreover, PS screening would support early diagnostic cardiac workup in order to decrease cardiovascular pain-related disability, poor health-related quality of life and reduce early death.

\section{P68}

AN INTERDISCIPLINARY APPROACH TO MOVING PATIENTS FORWARD IN MANAGEMENT OF CHRONIC PAIN

Joanne Stewart ${ }^{1}$, Jennifer Moore, Darlene Davis

${ }^{1}$ Capital District Health Authority Pain Services, Halifax, Nova

Scotia

POSTER AIM: All new patients referred to the Nova Scotia Capital District Health Authority (CDHA) pain clinics are invited to attend an educational program that includes an overview of pain management services available. By attending this program, known as "Moving Forward", patients are informed of wait times for clinic appointments and provided information on community and pain clinic resources that can enhance a self-management approach to managing chronic pain. The goal is for patients to complete a pain selfmanagement program(s) prior to their first pain clinic appointment.

METHODS: Facilitated by a pain clinic RN and Health Educator counselor, the program consisted of monthly, one hour drop-in educational sessions held at two different locations in Metro Halifax. A didactic method of teaching was utilized with time allotted at the end for questions. Sessions informed patients of chronic pain management philosophies, what to expect from the first pain clinic appointment, treatments and other strategies. A significant emphasis was placed on pain self-management programs. A telephone survey was part of the program evaluation. Ethics committee approval was not required for this project (considered part of ongoing QA).

RESULTS: 73 out of 107 participants responded to a telephone survey. Results demonstrated only a small number of participants became actively involved in self-management programs while waiting for their first clinic appointment.

CONCLUSIONS: Analysis of survey results will inform future practices, educational style and program content. 


\section{P69}

OPTIMIZING PAIN MANAGEMENT FOR THE HIGHLY OPIOID TOLERANT (H.O.T.) PATIENT HAVING SURGERY

Lynn Langille ${ }^{1}$, P Hammond, G Launcelott, D Milne, D Davis

${ }^{1}$ Capital District Health Authority, Halifax, Nova Scotia

POSTER AIM: The goal of this initiative is to improve perioperative pain management by developing a process for early identification of the highly opioid tolerant patient.

METHODS: Ethics Committee approval was not required as this project was considered a Quality Assurance initiative. A resource tool was created to assist pre-admission nurses and anesthesiologists in consistently identifying individuals who met the definition of high grade opioid tolerance. Once patients were identified, the Acute Pain Service Case Management Coordinator began a new process of completing a comprehensive pain assessment in the preoperative setting. Assessment findings formed the basis of a holistic pain management plan that included collaboration between nursing and anesthesiologist members of the Acute Pain Service. RESULTS: A consistent definition of high grade opioid tolerance among the multidisciplinary team. The adoption of the acronym H.O.T., (highly opioid tolerant) was established leading to further consistency with the definition and a familiarity with the initiative now known as the "H.O.T. project". The preoperative comprehensive pain assessment by APS nurses has allowed the patient to be engaged, set realistic goals and experience reduced anxiety through a clearer understanding of what to expect for pain management in the postoperative setting. Enhanced communication has resulted in an individualized pain management plan of care.

CONCLUSIONS: The collaboration among health care team members has resulted in multidisciplinary approach to opioid tolerant perioperative pain management. The results of this initiative support future research and strategies that provide better care to patients who meet the definition of highly opioid tolerant.

\section{P70 \\ PERCEIVED INJUSTICE AND RATINGS OF PAIN, DEPRESSION AND ANGER IN UNIVERSITY STUDENTS UNDERGOING AND EXPERIMENTAL PAIN INDUCTION PROCEDURE: SCALE DEVELOPMENT AND VALIDATION}

Esther Yakobov ${ }^{1}$, T Vrinceanu, MJL Sullivan

${ }^{1}$ McGill University, Montreal, Quebec

POSTER AIM: Perceived injustice in the chronic pain context has been conceptualized as an appraisal process reflecting the severity and irreparability of pain-related loss, blame, and unfairness. Research accumulated to suggest that perceived injustice is associated with greater pain severity, pain behavior, and mental health difficulties in individuals with various painful conditions. Research to date has proceeded from the assumption that perceptions of injustice arise from an incident, injury or illness that is associated with loss or suffering. However, perceptions of injustice may also reflect a trait-like dimension characterized by a propensity to experience stressful situations as a violation of justice principles. The aim of the present research was to validate a measure of perceived injustice in healthy individuals during an experimental pain induction procedure (cold water immersion).

METHODS: The study sample consisted of 70 (30 women, 40 men) healthy individuals recruited through advertisements on McGill University classifieds webpage. Participants were screened for medical conditions during a phone interview. Participants completed measures of perceived injustice, pain catastrophizing, and state mood questionnaires before and after the cold water immersion. During the 60 second immersion, participants were asked to rate their pain. Consenting participants were also videotaped during the cold water immersion with the use of two video cameras. Average water temperature was $5^{\circ} \mathrm{C}$.

RESULTS: The perceived injustice scale had high internal consistency $(\mathrm{r}=0.89 ; \mathrm{p}<0.001)$. It was found that individuals who scored high on a measure of perceived injustice also reported higher ratings of pain, anger, depression, anxiety, and pain catastrophizing. Marginal association between perceived injustice and protective pain behavior was also observed.

CONCLUSIONS: This was the first study to examine whether perceived injustice is associated with pain outcomes and negative mood states in healthy individuals. The results of the present study suggest that the propensity to perceive life experiences as unfair might underlie a trait-like characteristic present before the onset of pain, and influence how individuals interpret their painful condition.

\section{P71}

DEVELOPMENT OF AN ONLINE, THERAPIST-ASSISTED ADMINISTRATION OF ACCEPTANCE AND COMMITMENT THERAPY FOR CHRONIC PAIN

\section{Whitney Scott ${ }^{1}$, LM McCracken, L Forrester}

${ }^{1}$ King's College London, London, United Kingdom

POSTER AIM: Acceptance and Commitment Therapy (ACT) is associated with clinically meaningful improvements in function and quality of life among individuals with chronic pain. However, the provision of ACT for pain to date has predominantly been in the context of highly specialized tertiary care rehabilitation programmes, thus limiting its accessibility. Therefore, we have undertaken the development of a therapist-assisted Internet administration of ACT for chronic pain to improve its accessibility and cost-effectiveness.

METHODS: The treatment was developed on the basis of previous research on ACT for pain and the online psychological treatment delivery literature. To develop the treatment, three clinical psychologists with expertise in delivering ACT for pain worked in collaboration with a digital media team with extensive experience developing web-based interventions for the National Health Service in the United Kingdom.

RESULTS: All treatment materials will be provided via the 'No Delays' delivery platform. Patients will receive a standardized treatment package of 8 online sessions, which therapists can augment depending on patients' progress. Therapy sessions will take the form of video clips of therapists providing information and guiding patients through experiential exercises, metaphors, and behavioural activation strategies. Following each session, patients will respond to several questions assessing their in-session experiences; therapists will provide email feedback on this information prior to the next session. The content and delivery of the treatment were designed to be interactive and engaging, and to mimic face-to-face ACT for pain as much as possible. CONCLUSIONS: Plans for incorporating patient feedback into treatment refinement and for a small feasibility randomized-controlled trial will be discussed.

\section{P72 \\ GENETICS AND FIBROMYALGIA: STUDY ON THE RELATIONSHIP BETWEEN 5HT2A AND FIBROMYALGIA}

Tobore Onojighofia ${ }^{1}$, B Meshkin, D Schwarz, B Akindele, J Hubbard, Mary Knauer

${ }^{1}$ Proove Biosciences

POSTER AIM: The study objective is to determine if any association exist between genetics and risk of fibromyalgia.

METHODS: 185 subjects across 10 clinical research sites in the US. 94 diagnosed with Fibromyalgia (ICD9 codes 729.1 mean age (49), males (20), females (74),) and 91 controls matched for age, race and gender. Subjects were genotyped using Taqman ${ }^{\circledR}$ SNP Genotyping Assays (Life Technologies, Carlsbad, CA). It consists of a panel of 12 single nucleotide polymorphisms (SNPs) in genes encoding for proteins expressed in the mesolimbic reward pathway. These genes include: 5HT2a, 5-HTTL, COMT, ANKK1/DRD2, DRD1, DRD4, DAT, DBH, MTHFR, OPRK1, GABA-A receptor gamma2, and OPRM1.

RESULTS: A cross tab analysis using IBM SPSS v21 found significant association between only 5-HT2a (rs7997012) and FMS (5HT2a: Dominant Model (G/G vs. G/A-A/A) Pearson chi-square $\mathrm{p}=0.042$, twosided fisher's exact 0.044). Variants of 5-HT2A (G/A-A/A) were significantly more prevalent in subjects with FM compared to controls. Logistic regression found that $5 \mathrm{HT} 2 \mathrm{a}$ variants are more associated with FMS compared to the controls. $\mathrm{p}=0.043$, OR 1.899 .

CONCLUSIONS: This study suggests that 5-HT2a (rs7997012) may play a role in genetic predisposition to FM. Findings in this study will hopefully help further illuminate the role of genetics in FM as well as guide more rational and comprehensive pharmacological approach. 
P73

EVOLUTION OF ALLIED HEALTH AND NURSING ROLES THROUGH PROJECT ECHO ONTARIO PAIN: FROM CLINICAL CARE TO A MENTORSHIP MODEL

Amy Robidas ${ }^{1}$, DW Clark

${ }^{1}$ Toronto Rehab - University Health Netowrk, Toronto, Ontario

POSTER AIM: Chronic pain is recognised as a common and debilitating issue for approximately 20 percent of the Ontario population. Long waitlists, resource scarcity, and geographic challenges can mean patients do not have consistent access to pain specialists. Part of Ontario's Action Plan for Health Care includes giving primary care providers, including family teams, the tools to effectively treat and manage patients with chronic pain within their communities. Project ECHO Ontario Pain $(\mathrm{ECHO})$ is an innovative hub-and-spoke program whose goal is to deliver chronic pain management mentorship to health care providers across the province. The ECHO hubs are staffed by interprofessional teams with chronic pain expertise. In addition to physicians, our teams include a range of allied health and other health professionals, reflecting the evolution in health care toward a team based model of care. Prior to the rollout of ECHO, Toronto Rehab's interdisciplinary pain team provided direct complex pain care in a clinic setting.

\section{P74}

PAIN MANAGEMENT AND REDUCING LENGTH OF STAY IN TOTAL JOINT PATIENTS

\section{Kristine Desjardine ${ }^{1}$, A Rostas}

${ }^{1}$ Queensway Carleton Hospital, Ottawa, Ontario

POSTER AIM: The length of stay (LOS) for primary total hip and knee replacement patients has been steadily decreasing. In 2012 a decision was made to decrease the LOS to 2 days. A strategy was developed to involve all stakeholders and work together to implement the 2 day LOS pathway for total hip and knee. During the implementation process it became evident that patients were more nauseated and sedated on Intravenous patient controlled analgesia pain modality and contributed to a longer length of stay for some patients. A trial was done of the first 50 total joint patients to receive intrathecal and a peripheral nerve block. Previous pain modality practice from Department of Anesthesia was intrathecal and IV PCA.

METHODS: A strategy was developed to involve all stakeholders and work together to implement the 2 day LOS. Stakeholders included: surgeons, anesthesiologists, nursing, rehabilitation, diagnostic imaging, environmental services, nutrition services, social work and pharmacy. Meetings were held on a monthly basis to discuss progress and address barriers. The involvement of stakeholders guaranteed buy-in to the process. A new total joint replacement booklet for hips and for knees and a new clinical pathway was developed by nursing and rehabilitation staff with input from the other health disciplines. A new redesign of the post-operative anesthesia analgesia orders were done. Teaching tools for nursing and rehabilitation also needed to be changed. It was recognized that some patients would not be able to meet the 2 day LOS and would be pre identified in the rehabilitation sessions prior to surgery or by Social Work in the Pre-op Assessment Clinic.

RESULTS: Monthly monitoring shows that patients are less nauseated, less sedated, have improved function and mobility, able to meet the 2 day length of stay for discharge, improved discharge time (earlier in the day) and adequate and improved pain management.

CONCLUSIONS: The 2 day LOS clinical pathway was implemented Spring 2013. The changes to the pain modality and department of anesthesia pain orders for total joint patients occurred in the Fall 2014. Audits completed demonstrate that reducing length of stay and proper pain management ensures patient centered care. Other barriers needed to be addressed are: the timing of post-op X-rays; housecleaning of patient rooms; timing of meal service; and social work flow pre-op.

\section{P75}

MALE-SPECIFIC CONDITIONED PAIN HYPERSENSITIVITY IN MICE AND HUMANS

Loren J Martin ${ }^{1}$, EL Acland, AD Chen, B Kadoura, S Mirali, E Corley, S Tohyama, W Gandhi, P Schweinhardt, JS Mogil

${ }^{1}$ McGill University, Montreal, Quebec

POSTER AIM: Stress is known to induce analgesia or hyperalgesia depending on its nature, intensity and duration. In particular, repeated exposure to stressful stimuli results in stress-induced hyperalgesia (SIH) and produces maladaptive neurobiological changes. However, it is not known how the stress of a painful experience and the environment interact to produce conditioned changes in pain sensitivity.

METHODS: In this study, we administered a tonic pain stimulus to mice and people and measured their responses to acute thermal stimuli. In mice and people, we observe hypersensitivity to acute thermal nociception in the absence of tonic pain when subjects are returned, twenty-four hours later to an original environment in which they received a mild tonic pain. RESULTS: Surprisingly, conditioned thermal hyperalgesia was only evident in male subjects of both species and not females. In male mice, the conditioned thermal hyperalgesia was absent in castrated male mice and could be abolished by blocking the hypothalamic-pituitary axis before reexposure to the original context. Interestingly, male subjects re-exposed to the original testing environment displayed increased behavioral and neurochemical measures of stress. In male mice, the amount of pain behavior induced by the tonic pain stimulus correlated with measures of stress when they were re-exposed to the original tonic pain environment.

CONCLUSIONS: These results are in line with the concept that organisms become sensitized to their environment when they have had an aversive experience within that environment and suggest that this sensitization can persist. This demonstrates that the memory of the environment, or context, contributes to increased pain sensitivity observed on the following day and is likely driven through a combination of testosterone and stress hormones.

\section{P76}

THE PAIN OF OTHERS: EMPATHY'S INFLUENCE ON ANXIETYAND DEPRESSION-LIKE BEHAVIOUR IN FEMALE MICE

Sioui Maldonado-Bouchard ${ }^{1}$, A Klein, JS Mogil

1Department of Psychology, McGill University, Montreal, Quebec

POSTER AIM: The aim of this study is to identify the behavioural and physiological effects of long-term exposure to a familiar conspecific experiencing chronic pain. To do so, the anxiety- and depression-like behaviour and pain behaviour of mice housed in dyads - in which either both, one, or neither have undergone spared nerve injury (SNI) —-were compared.

METHODS: Adult CD-1 mice (7-13 weeks old), female and male, were used. Experimental conditions included both female and male same-sex pairs: $\mathrm{SNI}+\mathrm{SNI}$, sham + SNI, and sham + sham. At arrival, the mice were housed two per cage. No earlier than one week following arrival, baseline behavioural measures were collected. Two weeks following arrival, subjects underwent SNI surgery or sham surgery. Standard, validated tests of anxiety- and depression-like behaviour were conducted with all subjects 2 weeks following surgery, and then at 3-week intervals until the end of the experiment, at 3 months post-surgery. Anxiety-like behaviour was assessed by examining center activity in an open field, and time spent in the open arms of an elevatedplus maze. To assess depression-like behaviour, sucrose preference in the home cage, and immobility in the forced swim test were assessed. In addition, tactile sensitivity was assessed with von Frey filaments using the up-down method. Two (sex) $\times 2$ (housing condition) ANOVAs were conducted.

RESULTS: Female (but not male) shams housed with an SNI conspecific showed decreased activity in the center of an open field 7 weeks after surgery relative to baseline, whereas shams housed with a sham conspecific did not. Seven weeks after surgery, female (but not male) shams housed with an SNI conspecific also spent more time in the closed arms of an elevated plus maze, relative to shams housed with sham conspecifics. No differences were found on measures of sucrose preference or tactile sensitivity across housing conditions. Forced swim test performance and physiological correlates of anxiety and depression-like behaviour will be collected at the end of the experiment. Serum will be collected to examine levels of corticosterone as well as cytokines and chemokine via ELISAs. 
CONCLUSIONS: The current data suggest long-term exposure to a conspecific experiencing chronic pain differentially affects females, resulting in increased anxiety-like signs. In humans, providing long-term care for a relative suffering from a chronic illness, including chronic pain, is associated with depression and anxiety. Beyond the consciously perceived burden and psychological stress experienced by caregivers, the chronic exposure to a relative in pain may contribute to decreased well-being by pre-cognitive mechanisms. The next step will be to identify the mechanism via which exposure to a conspecific experiencing chronic pain modulates behaviour.

\section{P77}

THE FINE LINE BETWEEN GAIN AND LOSS: A GENETIC CHARACTERIZATION OF P2RX7 AND ITS RELEVANCE TO PAIN

Katerina S Lichtenwalter ${ }^{1}$, RN Lichtenwalter, AR Ase, M Niu, P Séguéla, S Komarova, GD Slade, R Dubner, RB Fillingim, JD Greenspan, RK Ohrbach, C Knott, BS Weir, W Maixner, LB Diatchenko

${ }^{1}$ McGill University, Montreal, Quebec

POSTER AIM: P2X7 is a purinergic receptor involved in the pro-inflammatory response. Its cellular function is two-fold: opening of a cation channel and recruitment of pannexin-1 to give entry to large molecules. P2X7 has been implicated in physiological and pathological conditions such as inflammation, bone tissue remodeling, tumourigenesis, depression, and pain. As a highly polymorphic gene, P2RX7 is an attractive target for genetic association studies, especially given the dichotomy between its gain-of-function and loss-of-function SNPs and their divergent roles in pain sensitivity.

METHODS: To refine these roles, we genotyped all P2RX7 missense SNPs with a minor allele frequency $\geq 0.01$ in a cohort of 1,082 chronic cases of temporomandibular disorder (TMD) and 2,144 TMD-free controls in the OPPERA (Orofacial Pain: Prospective Evaluation and Risk Assessment) project. Both groups underwent clinical examination and quantitative sensory testing to evaluate sensitivity to thermal, cutaneous, and deep pressure pain. A recent GWAS on the same cohort further provided us with a comprehensive coverage of the entire P2RX7 gene locus. To determine the effect of all missense polymorphisms tested, we introduced each mutation by site-directed mutagenesis and assayed changes in function using calcium imaging and electrophysiology. We further did regression analysis to uncover associations between individual SNPs and haplotype-informed groups of SNPs and tested phenotypes.

RESULTS: Gain-of-function rs208294 is positively associated with antihistamine consumption. Loss-of-function rs7958316 is negatively associated with IBS, while loss-of-function rs2230913 and rs7958311 are negatively associated with chronic pain.

CONCLUSIONS: A hyperactive P2X7 increases susceptibility to chronic pain and inflammation.

\section{P78}

\section{COMPLETE REVERSAL OF NEUROPATHIC AND} INFLAMMATORY ALLODYNIA IN PREGNANT MICE

\section{Sarah Rosen ${ }^{1}$, A Chen, J Austin, JS Mogil}

${ }^{1}$ McGill University, Montreal, Quebec

POSTER AIM: We have recently observed that administration of intrathecal glial inhibitors only reverse neuropathic and inflammatory mechanical allodynia in male mice, and have no effect in female mice. We have collected evidence suggesting that whereas male mice employ a microglia-dependent mechanism to mediate allodynia, and female mice instead employ $\mathrm{T}$ cells to the same ends. A possible explanation for this sex difference is that males and females have different $T$ cell immune environments: males have a Th2-dominant and females a Th1-dominant immune environment. However, during pregnancy, due to changes in sex hormone levels, females shift to a Th2-dominant immune environment. We wished to investigate the implications of this for microglia-dependence of mechanical allodynia.

METHODS: We used spared nerve injury as a model of neuropathic pain, and complete Freund's adjuvant as a model of inflammatory pain. In addition, all mechanical thresholds were measured using Von Frey filaments. Drugs were administered intrathecally, using a hamilton syringe. Administration of hormones was through an Alzet osmotic pump.

RESULTS: We show here that pregnant female mice do indeed switch to a microglia-dependent mechanism to mediate allodynia. In addition, we observe that in late pregnancy female mice that once suffered from a neuropathic injury have little to no evidence of pain. Female mice with spared nerve injury (SNI) or injected with complete Freund's adjuvant (CFA) progressively lose mechanical allodynia during pregnancy; shortly after delivery the mechanical allodynia returns to pre-pregnancy levels.

CONCLUSIONS: This phenomenon has been reported clinically, but has never been studied in animal models. Current experiments are examining the role of gonadal hormones, T-helper cells, and opioid receptors in these phenomena. Here we show that T-cell deficient, nude pregnant mice do not exhibit a blockade of mechanical allodynia during late pregnancy after SNI, suggesting that T-cells are playing a significant role in the phenomenon.

\section{P79}

\section{SEX DIFFERENCES IN THE INVOLVEMENT OF SPINAL P2X4 RECEPTORS AND BDNF IN PAIN HYPERSENSITIVITY INDUCED BY PERIPHERAL NERVE INJURY}

Josiane CS Mapplebeck ${ }^{1}$, S Beggs, JS Mogil, MW Salter

${ }^{1}$ The Hospital for Sick Children, Toronto, Ontario

POSTER AIM: Chronic neuropathic pain is characterized by mild to severe pain and results in significant human suffering and economic burden. The spinal mechanisms underlying neuropathic pain have been extensively investigated in male rodents, indicating an essential role for $\mathrm{P} 2 \mathrm{X} 4$ receptordriven release of brain-derived neurotrophic factor (BDNF) from spinal microglia in the maintenance of pain hypersensitivity. However, we recently demonstrated that microglia do not mediate pain hypersensitivity in female mice. The role of BDNF in mediating pain hypersensitivity in females remains unknown. Consequently, we investigated the role of spinal BDNF in neuropathic pain in female and male mice.

METHODS: Neuropathic pain was modeled in mice via spared nerve injury. Mechanical sensitivity was measured using von Frey fibers. Drugs were administered via intrathecal injection.

RESULTS: First, we found that intrathecal application of BDNF $(0.5 \mu \mathrm{g} /$ mouse $)$ in naïve mice induces pain hypersensitivity in either sex. Second, we determined that pain hypersensitivity after spared nerve injury was reversed in males but not females through inhibition of TrkB functioning via spinal application of TrkB-Fc $(0.5 \mu \mathrm{g} \times 3$ days $)$ and y1036 $(5.0 \mu \mathrm{g})$. Furthermore, we found that inhibiting P2X4 receptors with TNP-ATP $(5.0 \mu \mathrm{g})$ reversed pain hypersensitivity in males but not in females.

CONCLUSIONS: Our experiments provide evidence indicating that female mice do not use P2X4 receptors or BDNF to mediate neuropathic pain hypersensitivity. Taking into consideration sex differences in the spinal mediation of chronic pain may greatly improve future treatment development.

\section{P80}

\section{OPIOID BLOCKADE DECREASES SUBJECTIVE RATINGS OF RELIEF}

Rebecca Price ${ }^{1}$, W Gandhi, M-E Hoeppli, S Becker, P Schweinhardt ${ }^{1}$ McGill University, Montreal, Quebec

POSTER AIM: To determine whether the endogenous opioid system contributes to pain relief.

METHODS: Thirteen healthy volunteers completed two testing sessions, one with placebo and one with the opioid antagonist, naltrexone. In each session, a $3 \times 3 \mathrm{~cm}$ area of $0.4 \%$ capsaicin cream was applied to the forearm. After $20 \mathrm{~min}$, the cream was removed and a $3 \times 3 \mathrm{~cm}$ thermode (Pathway, Medoc, Israel) was applied to the area. Using individualized temperatures, participants then adjusted the applied warm temperatures to maintain a constant sensation at two target intensities: 170 and 195. They then rated the unpleasantness and average intensity of the painful stimulation. Following the painful stimulation, the temperature decreased to $25^{\circ} \mathrm{C}$, during which time participants either rated relief or pleasantness. Participants completed 2 relief and 2 pleasantness trials at both 170 and 195. Data were analyzed using paired sample t-tests. 
RESULTS: There were no significant differences between unpleasantness ratings, average intensity ratings or applied temperatures during either 170 or 195 trials (all p values $>0.1$ ). During 170 trials, naltrexone significantly decreased ratings of relief $(p=0.038)$ and pleasantness $(p=0.015)$ when the cool temperature was applied. Similarly, naltrexone significantly decreased ratings of relief $(p=0.004)$ and pleasantness $(p=0.007)$ during 195 trials when the cool temperature was applied.

CONCLUSIONS: Despite a non-significant effect on pain, naltrexone decreased ratings of relief and pleasantness during a relieving stimulus.

\section{P81}

A CRITICAL APPRAISAL AND GRADE ANALYSIS OF RCTS OF HYPNOTHERAPY FOR THE TREATMENT OF CHRONIC PAIN Jeffrey H Ennis ${ }^{1}$, K Poole, GL Ennis

${ }^{1}$ McMaster University Medical Centre, Hamilton, Ontario

POSTER AIMS: A 2014 metaanalysis found that hypnotherapy does reduce chronic pain. The authors asked, "How good is the data supporting the findings of this metaanalysis?"

METHODS: A review of literature in Medline, Embase, Psychoinfo Abstract, Proquest, and the Cochrane Institute database was conducted. Only RCTs with hypnotherapy as the intervention and pain reduction as the primary outcome were included,. The analysis involved the Critical Appraisal Methods(CAM) of studies and the GRADE (GR) analysis for the body of research evidence. Each author analyzed the papers with the final appraisal being constructed by discussion amongst the authors until a consensus was reached.

RESULTS: In total, there were 6 RCTs selected. CAM indicated that important measures of function and psychopathology were missing from most papers. Some studies had contaminated controls. The final GR score attained was a 2 out of a maximum of 4 indicating that the quality of the trials upon which the meta-analysis was based, was poor.

CONCLUSIONS: Current research suggests that hypnotherapy is efficacious in the treatment of CNCP. However, the quality of this research is suspect. Future research should pay attention to research design. Controls cannot be contaminated. It is vital that functional status and psychopathology are included as outcomes in these RCTs. This will help to shed light on the efficacy of hypnotherapy for the treatment of CNCP.

\section{REFERENCES}

1. Adachi T, Fujino H, Nakae A, Mashimo T, Sasaki J. A meta-analysis of hypnosis for chronic pain problems: A comparison between hypnosis, standard care, and other psychological interventions. Int J Clin Exp Hypnosis. 2014:62(1):1-28

2. Guyatt GH, Sackett DL, Cook DJ. Users' guides to the medical literature. II.How to use an article about therapy or prevention. B. What were the results and will they help me in caring for my patients? JAMA 1993;271:59-63.

3. Guyatt G, Oxman AD, Schünemann HJ, Tugwell P, Knottnerus A. GRADE guidelines: A new series of articles in the Journal of Clinical Epidemiology. J Clin Epidemiol 2011;64:380-382.

\section{P82}

THE CHRONIC PAIN MYTH SCALE: A NEW FRENCHCANADIAN INSTRUMENT MEASURING KNOWLEDGE, BELIEFS AND ATTITUDES OF THE GENERAL POPULATION REGARDING CHRONIC PAIN, ITS IMPACTS AND ITS TREATMENT

Anaïs Lacasse $^{1}{ }^{1}$ J-A Connelly, $M$ Choinière

${ }^{1}$ Université du Québec en Abitibi-Témiscamingue, Quebec

POSTER AIMS: Develop and validate a new French-Canadian scale measuring knowledge, beliefs and attitudes of the general population regarding chronic pain $(\mathrm{CP})$.

METHODS: The Chronic Pain Myth Scale (CPMS) preliminary items were developed based on different information sources: consultation of healthcare professionals and pain experts, review of existing measurement scales designed for patients or healthcare professionals, and patients' educational and awareness instruments and websites. Items were reviewed by pain experts for content validity and were pretested among individuals from the general population. The final CPMS contained 44 items, which are rated on 5-point Likert scales allowing the calculation of a total score (44-220) and three subscales scores: beliefs regarding people suffering from $\mathrm{CP}$, impacts of $\mathrm{CP}$, and treatment of CP (higher scores represent more positive attitudes). To establish CPMS's psychometric properties, it was administered to 1958 participants in the context of a web-based cross-sectional survey conducted among the general Quebec population.

RESULTS: The CPMS and its subscales showed adequate internal consistency $(\alpha=0.76-0.88)$. There was a statistically significant difference in CPMS scores between participants suffering and not suffering from CP (183.3 vs $179.7 ; \mathrm{p}<0.0001)$ and between those who were or were not healthcare professionals ( 189.6 vs $180.9 ; \mathrm{p}<0.0001$ ), which supports the construct validity of the scale (known-groups technique).

CONCLUSIONS: The description of knowledge, beliefs and attitudes of the general population regarding CP, its impacts and its treatment is important to better plan awareness and education activities. Our results support the internal consistency and the construct validity of the CPMS which could be used for such purposes.

\section{P83}

\section{KNOWLEDGE, BELIEFS AND ATTITUDES ABOUT CHRONIC PAIN, ITS IMPACTS AND ITS TREATMENT: A CROSS-SECTIONAL SURVEY OF THE QUEBEC GENERAL POPULATION}

Anaïs Lacasse $^{1}$, M Choinière, J-A Connelly

${ }^{1}$ Université du Québec en Abitibi-Témiscamingue, Quebec

POSTER AIMS: This study aimed to measure knowledge, beliefs and attitudes of the general population regarding chronic pain (CP), its impacts and its treatment.

METHODS: A web-based cross-sectional survey was conducted among the Quebec population between May and June 2014. Many diffusion platforms were used such as emails, social media, websites, and ads in newspapers.

RESULTS: A total of 1958 participants responded. Participants' mean age was $49.1 \pm 13.1$ years (range: $18-83$ ) and $78.1 \%$ were women. The majority reported suffering from $\mathrm{CP}(70.9 \%)$ and $13.7 \%$ were healthcare professionals (HCP), i.e. nurses, physiotherapists, physicians, psychologists, or pharmacists. Almost half of participants were not aware that CP affects approximately 1 out of 5 adults (43.5\%). A non-negligible proportion of participants agreed with the following statements:

- People with CP become dependent to their medications such as drug addicts (CP patients: $14.3 \%$, No CP: 23.1\%, HCP: 16.4\%),

- The treatment of CP is in the hands of medical professionals and not those of the patient (CP: 7.5\%, No CP: 6.64\%, HCP: 4.5\%),

- Consulting a psychologist is useless unless the person with CP is depressed (CP: 18.0\%, No CP: 14.4\%, HCP: 7.49\%).

Only $52 \%$ of people with CP were aware that they can obtain information and support from patients associations.

CONCLUSIONS: Our results suggest that the frequency of CP is not well known, and that negative attitudes are maintained in the population. An improved recognition of CP as well as a heighten awareness level and increased education for patients, healthcare professionals, and the general public is desirable.

\section{P84}

\section{TRANSLATIONAL CONTROL OF NOCIEPTION VIA 4E-BINDING PROTEIN 1}

A Khoutorsky, RP Bonin, RE Sorge, C Gkogkas, SM Jafarnejad, MH Pitcher, T Alain, L Perez-Sanchez ${ }^{1}$, L Martin, Y De Koninck,

F Cervero, JS Mogil, N Sonenberg

${ }^{1}$ McGill University, Montreal, Quebec

POSTER AIM: Activation of the mammalian target of rapamycin (mTOR) kinase in models of acute and chronic pain is strongly implicated in mediating enhanced translation and hyperalgesia; however, the molecular mechanisms by which mTOR regulates nociception remain unclear. Here we examined the role of mTOR main downstream effector, the eukaryotic initiation factor 4E-binding protein 1 (4E-BP1), in nociception. 
METHODS: We used biochemical, electrophysiological and behavioural techniques to study nociception in 4E-BP1/2 knockout mice.

RESULTS: Here we show that deletion of the 4E-BP1, a major mTOR downstream effector, acting by repressing eIF4E activity and cap-dependent translation, leads to mechanical, but not thermal pain hypersensitivity. Mice lacking 4E-BP1 exhibit enhanced translation of neuroligin 1, a cell-adhesion postsynaptic protein regulating excitatory synapse function, and show increased excitatory synaptic input into spinal neurons, and a lowered threshold for induction of synaptic potentiation. Pharmacological inhibition of eIF4E activity or genetic reduction of neuroligin 1 levels normalizes the increased excitatory synaptic input and reverses mechanical hypersensitivity.

CONCLUSIONS: Translational control by 4E-BP1 downstream of mTOR regulates the expression of neuroligin 1 and excitatory synaptic transmission in the spinal cord, and thereby contributes to enhanced mechanical nociception.

\section{P85}

\section{A META-REGRESSION ANALYSIS OF PLACEBO RESPONSE IN CLINICAL TRIALS OF NEUROPATHIC PAIN}

Alexander H Tuttle ${ }^{1}$, S Tohyama, T Ramsay, J Kimmelman,

P Schweinhardt, GJ Bennett, JS Mogil

${ }^{1}$ McGill University, Montreal, Quebec

POSTER AIM: Increasingly, promising new pharmacological targets for treating neuropathic pain are unable to pass randomized controlled drug trials, calling into question whether placebo-controlled randomized trial (RCT) design may in part explain trial outcome. In an effort to determine if placebo effects in neuropathic pain RCTs are increasing over time, we conducted a literature review of published neuropathic pain placebo-controlled randomized clinical trials.

METHODS: Using EMBASE, Medline, and Cochrane online databases. Specifically, a search for "neuropathic pain" and "drug," limited to randomized clinical trials, yielded a combined total of 1793 articles. We also included an additional 106 articles from a similar meta-analysis conducted by Finnerup et al. (2005), bringing our article total to 1899. First-pass inclusion criteria included: English language only, randomized controlledtrials, neuropathic pain conditions (except for migraine, trigeminal neuralgia, or orofacial pain), systemic drugs only, visual analogue scales or numeric rating scales, and parallel or cross-over trial design. After first-pass screening, 245 articles were included for additional analysis. After secondpass screening, a total of 85 articles were included in our final data set.

RESULTS: Analysis of reported pain measures extracted from placebo and treatment groups yielded several striking correlations between study design and reported placebo-group pain scores. First, placebo ratings (reductions in pain measures) are becoming increasingly stronger over time. However, multiple regression analysis indicates that the size of the study, number of treatment arms, and study duration/number of site visits are the driving correlative factors predicting an increase in placebo response. Finally, the magnitude of the placebo response appears to be limited to North American studies.

CONCLUSIONS: Taken together, our findings are similar to those reported in both the depression and schizophrenia literature. In essence, changes in the magnitude of the placebo response appear to be increasing in more recent studies. However, it is important to note that drug responses are also increasing over time, indicating that the same study design factors may be similarly impacting patient response across trial conditions.

\section{P86}

TOPICAL MANNITOL REDUCES CAPSAICIN-INDUCED PAIN: RESULTS OF A PILOT LEVEL, DOUBLE-BLIND RANDOMIZED CONTROLLED TRIAL

Helene Bertrand ${ }^{1}$, M Kyriazis, KD Reeves, J Lyftogt, D Rabago

${ }^{1}$ University of British Columbia, Vancouver, British Columbia

POSTER AIM: To assess the effects of a mannitol-containing cream in a capsaicin- based pain model.

METHODS: Capsaicin $0.075 \%$ cream was applied to both halves of each participant's upper lip, inducing pain via stimulation of the transient receptor potential vanilloid one (TRPV1, capsaicin) receptor, then removed after five minutes, or when participants reported a burning pain of $8 / 10$, whichever came first. A cream containing mannitol and the same cream without mannitol (control) were then immediately applied, one on each side of the lip, in an allocation-masked manner. Participants self-recorded a numerical rating scale (NRS, 0-10) pain score for each side of the lip per minute for 10 minutes. A t-test was performed to evaluate the pain-score change from baseline between each side of the lip at each recording. Area under the curve (AUC) analysis was used to determine the overall difference between groups.

RESULTS: Participants reached a capsaicin-induced pain level of $7.8 \pm 1.0$ points in $3.3 \pm 1.6$ minutes, that was equal on both sides of the lip. Both groups reported progressive diminution of pain over the 10-minute study period. However, participants reported significantly reduced pain scores on the mannitol half-lip compared to control at 3 through 10 minutes $(\mathrm{p}<0.05)$, and in AUC analysis $(\mathrm{p}<0.001)$.

CONCLUSIONS: Mannitol cream reduced self-reported pain scores in a capsaicin pain model more rapidly than a control cream, potentially via a TRPV1 receptor effect.

\section{P87} BENEFIT OF DEXTROSE PROLOTHERAPY IN PAINFUL
ROTATOR CUFF TENDINOPATHY CASES RECEIVING
PHYSICAL THERAPY: A RANDOMIZED CONTROLLED TRIAL PHYSICAL THERAPY: A RANDOMIZED
Helene Bertrand
', D Reeves, C Bennett ${ }^{1}$ University of British Columbia, Vancouver, British Columbia

POSTER AIM: Rotator cuff tendinopathy (RoCT) is a common, often painful, progressive degenerative condition. Prolotherapy is an injection therapy for chronic musculoskeletal pain. Our objective was to assess the efficacy of concomitant prolotherapy in participants with painful RoCT who receive staged physical therapy.

METHODS: Three arm, blinded (injector [partial], assessor, injection group participants) randomized controlled trial. Seventy four participants with chronic shoulder pain and ultrasound-confirmed rotator cuff tendinopathy. Six therapy sessions along with three, monthly, injection sessions of either painful entheses with $0.1 \%$ lidocaine $/ 25 \%$ dextrose (Entheses Dextrose Group), painful entheses with $0.1 \%$ lidocaine/saline (Enthesis Saline Control), or to $1 / 2$ inch depth above the painful enthesis with $0.1 \%$ lidocaine/saline (Superficial Saline Control). The primary clinical measure was a $0-10$ VAS or Numerical Rating Scale for pain. Satisfaction with treatment was measured on a $0-10$ scale.

RESULTS: Fifty percent or more (50-56\%) of each therapy plus injection group improved $\geq 2$ in their VAS pain score at short term ( 3 month) follow up with no difference between groups $(p=0.909)$. Seventy percent $(19 / 27)$ of Enthesis Dextrose recipients maintained a pain improvement of $>2.0$ at 9 months, compared to $50 \%(10 / 20 ; p=0.132)$ of Enthesis Saline recipients and $35 \%(9 / 26 ; p=0.009)$ of Superficial Saline recipients. Enthesis Dextrose recipients were more satisfied $(\mathrm{p}=0.009)$ than Superficial Saline recipients. Only $8 \%(6 / 73)$ participants were both confident and correct about the type of injection they received.

CONCLUSIONS: Physical therapy plus dextrose prolotherapy resulted in more sustained pain improvement and satisfaction in participants with painful rotator cuff tendinopathy than physical therapy plus superficial saline injection, with intermittent results for the entheses saline injection. 


\section{P88}

DEVELOPMENT OF AN INTEGRATED ONLINE DATABASE (NOPAIN) FOR ACUTE PAIN MANAGEMENT SERVICES A Bhatia ${ }^{1}$, A Buzon-Tan, S Walker, K Kirkham, D Ilangomaran, B Yau, S Alvi, R Brull

${ }^{1}$ Toronto Western Hospital, University of Toronto, Toronto, Ontario

POSTER AIM: Acute Pain management Services (APS) were introduced several decades ago in our institution, and the scope of services has expanded to include provision of analgesia to pre-operative, non-operative, and postoperative patients. A paucity of affordable, commercially available APS database options limit the ability to capture and explore the patient analgesic experience, adverse effects, as well as improve patient safety. Institution specific, locally developed APS paper forms have been utilized but can be unreliable due to gaps in data capture and inconsistent use of measurement tools.

METHODS: NOPAIn (Networked Online Processing of Acute Pain Information) database was built on Filemaker Pro. Prospective, realtime pain-related data is recorded and accessed using handheld devices, but can easily be accessed from any networked computer. Data is captured in a standardized form and is readily accessible to staff. Drop down menus, at-a glance summaries of follow up visits, and ability to print chart-ready follow-up visit summary labels wirelessly are unique features of this database.

RESULTS: NOPAIn has been in used for over 800 patients at our hospital. Its development has required less than one-tenth of the cost quoted by commercial vendors. NOPAIn offers several advantages over commercial options - adaptability to local needs, ease of modification, and lack of "tiein" to vendors. Measurement tools for analgesic efficacy (numerical rating scale) and capture of adverse effects are applied consistently. Billing reports may also be generated.

CONCLUSIONS: Analysis of the NOPAIn searchable database using advanced statistical techniques may help identify factors influencing analgesic success and risk factors for adverse events.

\section{P89}

BARIATRIC SURGERY AND PAIN: ARE WE DOING THE RIGHT THING?

$\underline{\text { P Kastanias }}^{1}$, S Robinson, F Paat, W Wang, C Lopez, A Buzon-Tan ${ }^{1}$ University Health Network, Toronto Western Hospital, University of Toronto, Toronto, Ontario

POSTER AIM: The purpose of this study is to describe the frequency, incidence and severity of acute, abdominal postoperative pain as well as the incidence and prevalence of chronic pain in the TWH Bariatric surgery population. Studies have shown that surgical weight loss intervention is an effective tool in treating obesity and improving obesity-related medical comorbidities. The University of Toronto Collaborative Bariatric surgery program performs two types of laparoscopic bariatric surgeries: Roux-en-Y gastric bypass and vertical sleeve gastrectomy. There are several inherent challenges with providing effective postoperative pain management in this patient population. Due to a paucity of literature in this area, further research is warranted in order to inform appropriate postoperative pain management strategies.

METHODS: This is a longitudinal, descriptive correlational study. Fifty consecutive Toronto Western Hospital (TWH) patients who have undergone bariatric surgery have been asked to complete the Brief Pain Inventory (BPI) preoperatively and at 6 months postoperatively. Verbal Numerical Pain Rating Scores, patient satisfaction with pain management, and analgesic use was obtained on postoperative day 0,1 , at discharge and 48-72 hrs and 6 months postoperatively. Data will analysed for demographic and dependent variable frequencies and correlations.

RESULTS: Preliminary results show a high degree of patient satisfaction with postoperative pain management, with a trend towards decreased rest and movement-related pain over time. A trend towards decreased average chronic pain severity and pain interference scores at 6 months postoperatively was noted.

CONCLUSIONS: It is anticipated that results will help to inform the assessment and management of acute and chronic pain in Bariatric Surgery patients. 


\section{A}

Acland EL ...................... P75

Agboatwalla M ................. P39

Ahmed S . . . . . . . . . . . . . . P P60,P61

Akindele B .............. P36,P72

Alain $\mathrm{T} \ldots \ldots \ldots \ldots \ldots \ldots \ldots . \ldots . \ldots 84$

Alvi S.................... P88

Arbeau K ..................... P50

Ase AR ................... P77

Ashly $G \ldots \ldots \ldots \ldots \ldots \ldots \ldots$. P2O

Atkinson $\mathrm{N} \ldots \ldots \ldots \ldots \ldots \ldots \ldots \ldots$. P3

Austin E .................. P52

Austin J ....................... P78

\section{B}

Badr HE . . . . . . . . . . . . . . . . . . P39

Bakhtadze M .................. P26

Ballantyne P ............. P39,P40

Becker S . ...................9C,P80

Bednarikova M ................ P26

Beggs S .................... P79

Bellingham GA ................. P5

Bennett C . . . . . . . . . . . . . . . P87

Bennett GJ . . . . . . . . . . . . . .9D,P85

Benoit B $\ldots \ldots \ldots \ldots \ldots \ldots \ldots$ P51,P56

Bent M .................. P15

Bernabeo D ................... P52

Bertrand $\mathrm{H} \ldots \ldots \ldots \ldots \ldots \ldots \ldots$ P86,P87

Bhatia A $\ldots \ldots \ldots \ldots \ldots$. . . . 13,13A,P88

Bherer $\mathrm{L} \ldots \ldots \ldots \ldots \ldots \ldots \ldots$. P46

Birnie KA $\ldots \ldots \ldots \ldots \ldots$ P21,P23,P25

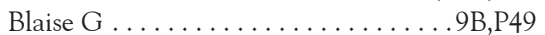

Boerner KE ................ P21,P34

Boire $\mathrm{G} \ldots \ldots \ldots \ldots \ldots \ldots \ldots \ldots$ P54

Boitor M ................... P42

Bolton T . .................. P30

Bonin RP . . . . . . . . . . . . . . P84

Borniard J . ...................... P24

Borowska A .................. P43

Bostick G ................18,18B

Bowry R ................... P15

Brose $\mathrm{K} \ldots \ldots \ldots \ldots \ldots \ldots \ldots . . \ldots$ P63

Brouillard P ................... P46

Brown S ...................... P41

Brull R .................... P88

Buckley DN ................... P28,P29

Buhle J..................... P46

Buna $D \ldots \ldots \ldots \ldots \ldots \ldots \ldots$ P7

Burns LC . . . . . . . . . . . . . . . . 9A,P18

Busse J .................. 31,31C

Busse JW ................. P28,P29

Buzon-Tan A . ................. P88,P89

$\mathrm{C}$

Caes $\mathrm{L} \ldots \ldots \ldots \ldots \ldots \ldots \ldots \ldots$. P21,P23

Cairns B $\ldots \ldots \ldots \ldots \ldots \ldots \ldots \ldots \ldots 24,24 B$

Cairns BE ................... P11

Calic M .................... P35

Campbell F . . . . . . . . . . . . . . P41,P64

Campbell L . . . . . . . . . . . . . . . . . . P8

Campbell-Yeo M …......16,23,P51,P56

Campos AA $\ldots \ldots \ldots \ldots \ldots \ldots$. . . . . 41

Cane D ................... P44,P45

Cantin L $\ldots \ldots \ldots \ldots \ldots \ldots \ldots \ldots$. $\ldots$ B, P49

Caprio M .................... P7

Card R . . . . . . . . . . . . . . . P63
Carr LK ....................... P13

Cervero $\mathrm{F} \ldots \ldots \ldots \ldots \ldots \ldots \ldots$ 11,29,P84

Chambers $\mathrm{C} \ldots \ldots \ldots \ldots \ldots$ 7,7C,P53

Chambers CT …......P21,P23,P25,P34

Chan B ................... P65

Chatterjee A ................... P4

Chen A . . . . . . . . . . . . . . . . P78

Chen AD ................... P75

Chen D ................... P30

Chin K ...................... P64

Choinière M ..... 9E,P32,P33,P37,P82,P83

Chorney J . . . . . . . . . . 32,32C,P25

Cicero TJ .................. P58

Clark AJ ............ 4,4A,P23,P31

Clark DW $\ldots \ldots \ldots \ldots \ldots \ldots \ldots$. P73

Clarke H $\ldots \ldots \ldots \ldots \ldots \ldots . .21,21$ B,P61

Clarke K .................... P15

Connelly J-A . . . . . . . . . . . . . . P82,P83

Constant J ................. 9B,P49

Cooper $\mathrm{L} \ldots \ldots \ldots \ldots \ldots \ldots$. A $, 25,25 \mathrm{~A}$

Cooper L . . . . . . . . . . . . . . . . P18

Corley E . . . . . . . . . . . . . P75

Corman J .........................19

Corman J ...................... 19C

Corsini-Munt $S \ldots \ldots \ldots \ldots \ldots \ldots, 6,6 \mathrm{C}$

Cowen J ..................... P30

Crawford J .................. P26

Cundiff $\mathrm{L} \ldots \ldots \ldots \ldots \ldots \ldots \ldots \ldots, \mathrm{P} 7$

\section{D}

Daigle $\mathrm{K} \ldots \ldots \ldots \ldots \ldots \ldots$. . . . . . . . 54

Dart R .................. P52

Dart RC ................. P58

Dasgupta N . . . . . . . . . . . . . P58

Davis D .................. P68,P69

Davis K . ............... 7,7A,17

Davis $W \ldots \ldots \ldots \ldots \ldots \ldots \ldots$ P7

De Koninck Y . . . . . . . . . . . . . . P84

de Vos C .................... P57

Deptuck D ................... P66

Descarreaux M ............... P55

Desjardine K ................ P74

Dewar A .................... P20

Diatchenko LB ................ P77

Disher T ............... P51,P56

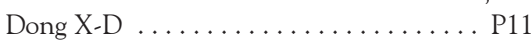

Downe PJ .................. P63

du Souich P . . . . . . . . . . . . . . 9B,P49

Dubin R . . . . . . . . . . . . . 8,8A,30

Dubin RE ................ P66

Dubner R . . . . . . . . . . . . . . . . . . P77

Dubois J-D ................. P55

\section{$\mathrm{E}$}

Ebrahim $\mathrm{S} \ldots \ldots \ldots \ldots \ldots \ldots \ldots \ldots$ P28

Eilayyan $\mathrm{O} \ldots \ldots \ldots \ldots \ldots \ldots \ldots$. . . . . . 60

Ellis MS .................... P58

Emanuele C.................. P65

Ennis GL ................... P81

Ennis $\mathrm{JH} \ldots \ldots \ldots \ldots \ldots \ldots$. 881

Evans RT ................... P38

\section{$\mathrm{F}$}

Fashler SR . . . . . . . . . . . 9,9A,P18

Fedoroff I . . . . . . . . . . . . . . P43

Fernandez CV ................. P25
Fillingim RB $\ldots \ldots \ldots \ldots \ldots \ldots \ldots$ P77

Finley $\mathrm{G} \ldots \ldots \ldots \ldots 10,10 \mathrm{C}, 20,22,22 \mathrm{~A}$

Fiola JL .................. P42

Forrester L . . . . . . . . . . . . . . . P71

Foster J . . . . . . . . . . . . . P65

French DJ ............... P38

Frost $\mathrm{A} \ldots \ldots \ldots \ldots \ldots \ldots \ldots . \ldots \ldots$

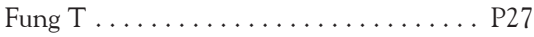

Furlan A ................30,30C

\section{G}

Galan JGN ................ P57

Gallant N .................. P50

Gandhi W .............9C,P75,P80

Garfield H . . . . . . . . . . P3,P8,P14,P35,P59

Gastaldo D ................ P22

Gazerani P ................. P11

Gelinas C .................. P42

Gennis $\mathrm{H} \ldots \ldots \ldots \ldots \ldots \ldots \ldots$. P8,P14

Geoffroy P . . . . . . . . . . . 19,19B

Girard-Tremblay L . . . . . . . . . . . . . P54

Gkogkas C . . . . . . . . . . . . . . P84

Goffaux P ................... P54

Goldberg L . . . . . . . . . . . . 9A,P18

Goodman W ................ P26

Gordon A .................. P9,P19

Grafstein E ................ P20

Green JL .................. P58

Greenberg S $\ldots \ldots \ldots$ P3,P8,P14,P35,P59

Greenspan JD ............... P77

Grosman-Rimon L . . . . . . . . . . . P61,P62

Gullickson C . . . . .................23,23B

Guo S-L . . . . . . . . . . . P16,P17

Gusztak R ................. P30

Guyatt $\mathrm{GH} \ldots \ldots \ldots \ldots \ldots \ldots . \ldots . \ldots 28$

Guzy $\mathrm{G} \ldots \ldots \ldots \ldots \ldots \ldots \ldots \ldots \ldots \ldots$

\section{$\mathrm{H}$}

Hagen NA .............. 9,9B,P49

Haller T . . . . . . . . . . . . . . . . . . .9B,P49

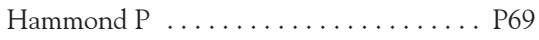

Hapidou EG . . ............... P29

Harris L ................... P41

Harrison $\mathrm{D} \ldots \ldots \ldots \ldots \ldots \ldots \ldots .7,7 \mathrm{~B}$

Hayden J ................... P53

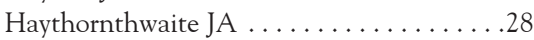

Higgins $\mathrm{K} \ldots \ldots \ldots \ldots \ldots \ldots \ldots . . \ldots \ldots \ldots \ldots$

Hildebrand M . ..............12,12C

Hodgson $\mathrm{N} \ldots \ldots \ldots \ldots \ldots \ldots \ldots . . . \ldots$. . . . . . . . . . . . . .

Hoeppli M-E . . . . .......... 9C,P80

Howell D . . . . . . . . . . . . . . . . P17

Hrkal P ...............26,26A

Hsu S . . . . . . . . . . . . . P28

Hubbard J ............... P36,P72

Hudson $\mathrm{H} \ldots \ldots \ldots \ldots \ldots \ldots \ldots$. P52

Huguet A .................... P53

\section{I}

Ilangomaran D . . . . . . . . . . . . . P88 Ip $G \ldots \ldots \ldots \ldots \ldots \ldots \ldots \ldots \ldots$ P15 Isaac $\mathrm{L} \ldots \ldots \ldots \ldots \ldots \ldots \ldots \ldots . \mathrm{P} 41, \mathrm{P} 64$

\section{$\mathrm{J}$}

Jafarnejad SM ................ P84 Jamison RN . . . . . . . . . . . . .2,2A Janati $A B \ldots \ldots \ldots \ldots \ldots \ldots \ldots \ldots \ldots$ 


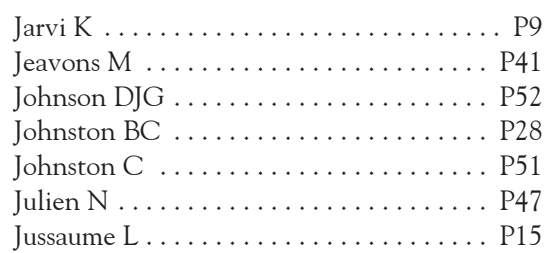

$\mathrm{K}$

Kadoura B .................. P75

Kallyth SM ............ 31,31B,P29

Kamaleldin M ................ P28

Kashin B .................. P15

Kastanias P. . . . . . . . . . . . . . . . P89

Katz J . . . . . . . . . . 9A,21,P18,P61,P64

Katz L . . . . . . . . . . . . . . . P13

Katzman JG . . ................30,30A

Kazazian V ................... P65

Kesiktas N . . . . ............... P26

Khan MU .................. P2

Khoutorsky A ................ P84

Kimmelman J . . . ...........9D,P85

Kinkade A . . . . . . . . . . . ..... P20

Kirkham K................. P88

Klein A . . ................. P76

Knauer M ...............P36,P72

Knott C . . . . . . . . . . . . . . P77

Komarova S . . . .............. P77

Korz W ................. 9B,P49

Krsmanovic A . . ........... 3,3B,P13

Kucyi A ..................17,17A

Kumbhare D .............. P61,P62

Kunz R ................... P28

Kushneriuk B ............... P30

Kyriazis M ................ P86

\section{$\mathrm{L}$}

Lacasse A $\ldots \ldots \ldots \ldots \ldots \ldots$. P47

Lacasse Aï ................ P82,P83

Ladak S . . . . . . . . . . . . . . . . 21,21A

Lakha SF ............. P4,P39,P40

Lalloo $\mathrm{C} \ldots \ldots \ldots \ldots \ldots \ldots \ldots$. 441

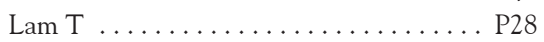

Langille $\mathrm{L} \ldots \ldots \ldots \ldots \ldots \ldots \ldots$. . . . . . . . . . . . . . . 99

Lapointe B . . . . . . . . . . . . . . . 98, P49

Latimer $\mathrm{M} \ldots \ldots \ldots \ldots \ldots \ldots . \ldots 22,22 \mathrm{~B}$

Lau S .................... P9

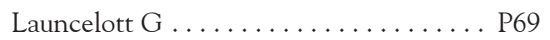

Lichtenwalter KS ............. P77

Lichtenwalter RN ............... P77

Liu C ..................... P50

Logan KJ ............... 20,20B

Lopes LC . . . . . . . . . . . . . . . . . P28

Lopez C . . . . . . . . . . . . . . . . P89

Luca $\mathrm{S} \ldots \ldots \ldots \ldots \ldots \ldots \ldots \ldots$. . . . . . . . . . 65

Lussier M . . . . . . . . . . . . . . . P46

Lyftogt J . . . . . . . . . . . . . P86

Lynch M ......... 8,8C,P23,P31

\section{M}

MacLeod BA ................ P27

Mailis A ................ P39,P40

Mailis-Gagnon A . . . . . . . . . . . P4 P

Maixner W .................. P77

Maldonado-Bouchard S . .......... P76

Mapplebeck JCS . . . . . . . . . . . . . . P79

Marchand S.................. P54

Marcon D ..................... P31
Marsden J .................. P20

Martin L . . . . . . . . . . . . . P84

Martin LJ . . . . . . . . . . . . . 23,23C,P75

Masetto A ................. P54

Mayer R................... P13

McCarthy M ............... P44,P45

McCracken LM . . . . . . . . . . . . . . P71

McDougall J . . ...............12,12A

McDougall JJ . . . . . . . . . . . . . . . .24,24A

McEwan R . . . . . . .............25,25B

McGillion M ........... 25,25C,P17

McGlynn M ...............30,30B

McGrath PJ . . . .............. P25,P53

McKay W.................. P30

McMurtry CM .......... 32,32B,P34

Medhian $\mathrm{H} \ldots \ldots \ldots \ldots \ldots \ldots$. P15,P19

Meshkin B ..............P36,P72

Milne D . . . . . . . .............. P69

Mirali $S \ldots \ldots \ldots \ldots \ldots \ldots \ldots$ P75

Mogil JS ... 9,9D,P75,P76,P78,P79,P84,P85

Moldwin RM . . . . . . .......... P13

Monticone M ................ P26

Montoya L . . . . . . . . . . . . . . . P28

Moore J .................... P68

Muere A .............. 3,3C,P13

Muir J ................. P20

Mulla S ........... 31,31A,P28,P29

Munro P ................... P20

Mustaneer $\mathrm{H} \ldots \ldots \ldots \ldots \ldots \ldots$ P9

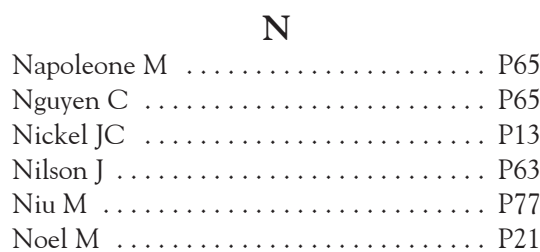

\section{$\mathrm{O}$}

O'Brien M .................... P11

O'Brien Munyao K ............... P22

O'Keefe-McCarthy S . . . . . . . P16,P67

O'Leary M . . . . . . . . . . . . . . . . . P66

O'Neill M ................22,22C

O'Neill MC . . . . . . . . . . . . . . . . P59

Ohrbach RK .................. P77

Olivier S . . ................. P24

Olivieri $\mathrm{L} \ldots \ldots \ldots \ldots \ldots \ldots \ldots \ldots \ldots$ P28

Ong-Lam M ........... 9B,P43,P49

Onojighofia T . . . . . . . . . . P36,P72

Oosenbrug E ............. 9A,P18

\section{$\mathrm{P}$}

Paat $F \ldots \ldots \ldots \ldots \ldots \ldots \ldots \ldots \ldots . . \ldots 89$

Page MG ................. P64

Pagé $G \ldots \ldots \ldots \ldots \ldots$ 9E,P32,P33

Pagé $\mathrm{MG} \ldots \ldots \ldots \ldots \ldots \ldots \ldots \ldots$. P37

Paneduro D ................. P9,P19

Parappilly B . . . . . . . . . . . . . . P20

Parkinson WL .................. P62

Peng P ..................13,13B

Pennefather $\mathrm{P} \ldots \ldots \ldots \ldots \ldots \ldots$. P39, $\mathrm{P} 40$

Perez J ............ 26,26C,P24,P48

Perez-Sanchez J . . . . . . . . . . . . P84

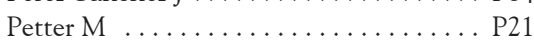

Phil D ................... 1,11

Phillips CRJ ................ P5

Piché M ................. P55
Pike $M \ldots \ldots \ldots \ldots \ldots \ldots \ldots . . . . . . .231$ Pillai Riddell R ......22,32,32A,P3,P6,P8, P14,P35,P59

Pinhasov A .................. P6

Pink L ................... P19

Pitcher MH . . . . . ............. P84

Poole K. . . . . . . . . . . . . . . . . P81

Prescott $\mathrm{S} \ldots \ldots \ldots \ldots \ldots \ldots \ldots . \ldots \ldots, 5 \mathrm{~A}$

Price $R \ldots \ldots \ldots \ldots \ldots \ldots$. . . 9 C,P80

Proudman J ................. P15

Puil E..................... P27

\section{R}

Rabago D . ................... P86 Racine $\mathrm{N} \ldots \ldots \ldots \ldots \ldots \ldots . \ldots 32, \mathrm{P} 14$

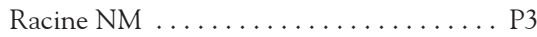

Rainville $\mathrm{P} \ldots \ldots \ldots \ldots \ldots \ldots . . \ldots . \ldots . \ldots$

Ramsay T . . . . . . . . . . . . . . . 9D,P85

Rancourt K . . . ..................6,6A

Razavi S . . . . . . . . . . 9A,P18,P64

Ready L . . . . . . . . . . . . . . . . . P67

Reeves D . . . . . . . . . . . . . . P87

Reeves KD . . . . . . . . . . . . . . . P86

Reid Jr MC $\ldots \ldots \ldots \ldots \ldots \ldots \ldots 2,2 \mathrm{~B}$

Riva JJ .................. P28

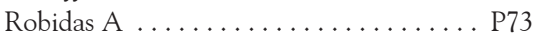

Robinson S. . . . . . . . . . . . . . P89

Rosen $\mathrm{NO} \ldots \ldots \ldots \ldots \ldots \ldots \ldots .6,6 \mathrm{~B}$

Rosen S . . . . . . . . . . . . . . . . P78

Rosenbloom BN ............... P64

Rostas A ....................................... 74

Roy $\mathrm{M} \ldots \ldots \ldots \ldots \ldots \ldots$ 17,17C,P46

Ruskin D .................. P41

\section{S}

Saidi H $\ldots \ldots \ldots \ldots . .99$ 9,9E,P32,P33,P37

Salter MW …........9F,P12,P79

Sangster M ...............20,20C

Sawhney M ................. P15

Sawynok J .................. P31

Schandelmaier S . .............. P28

Scheidecker A ................ P28

Schinkel M .................. P34

Schlosser T . ................. P63

Schwarz D ...............P36,P72

Schweinhardt P . . . . . 9C,9D,P75,P80,P85

Scott W . . . . . . . . . . . 11,18,18C,P71

Séguéla P . . . . . ............ 5,5B,P77

Sessler DI . . . . . . . . . . . . . . . P28

Setnik B .................19,19A

Severtson SG $\ldots \ldots \ldots \ldots \ldots \ldots$ P58

Shamji MF ........9,9F,13,13C,P1,P12

Shir $\mathrm{Y} \ldots \ldots \ldots \ldots \ldots \ldots \ldots 4 \mathrm{C}$

Shir $\mathrm{Y} \ldots \ldots \ldots \ldots \ldots \ldots \ldots \ldots . . \ldots \ldots$ P48

Sims $M \ldots \ldots \ldots \ldots \ldots \ldots \ldots \ldots \ldots \ldots \ldots$

Slade GD .................... P77

Smeenk T ................. P9

Smyth C ................26,26B

Sonenberg N . . . . . . . . . . . . . . P84

Songok J .................... P22

Sorge RE ................... P84

Spanswick C ..................4,4B

St-Amant H. . . . . . . . . . . . P P47

Stevens B .............. 10,10B

Stevens BJ .................. P22

Stewart J.................. P68

Stewart S . . . . . . . . . . . . . P50

Stinson J …... 2,2C,P23,P41,P64,P65 
Stinson JN . . . . . . . . . . . . . . . P53

Stone $\mathrm{L} \ldots \ldots \ldots \ldots \ldots \ldots \ldots . . \ldots 24,24 \mathrm{C}$

Sullivan MJL ................. P70

Sung YC $\ldots \ldots \ldots \ldots \ldots \ldots \ldots$ P17

\section{$\mathrm{T}$}

Tablon $\mathrm{P}$ . P6

Tabry $\mathrm{V} \ldots \ldots \ldots \ldots \ldots \ldots \ldots \ldots . . \ldots \ldots$

Thompson M ............... P52

Todorovic S . . . . . . . . . . . 12,12B

Tohyama $S \ldots \ldots \ldots \ldots$ 9D,P75,P85

Tougas ME .................... P53

Tracey I . . . . . . . . . . . . . . 1,11

Tripp D .................... 3, 3 A

Tripp DA . . . . . . . . . . . . . . . P13

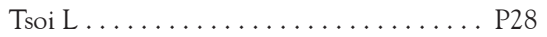

Tu YS . . . . . . . . . . . . . 9F,P12

Tupper S ................. P63

Tuttle AH .............. 9,9D,P85

Tyrrell J

P41

\section{V}

Vachon-Presseau E ................ 17,17B

Vandersteen A .............20,20A

Vandvik PO ................ P28

Van-Vlack CK ................ P66

Vernon $\mathrm{H} \ldots \ldots \ldots \ldots \ldots \ldots \ldots . . . . . . . .26$

Visca $R \ldots . \ldots \ldots \ldots \ldots \ldots$. . . . . . 60

Vrinceanu $\mathrm{T} \ldots \ldots \ldots \ldots \ldots \ldots . . . . . . .270$

\section{W}

Walker S. ................... P88

Wang A .................... P30

Wang W........................ 889

Ware M ....... 9E,P32,P33,P37,P47,P60

Ware MA $\ldots \ldots \ldots \ldots \ldots \ldots 8,8 \mathrm{~B}, 11$

Watt-Watson J ............ 10,10A,P17

Waxman JA $\ldots \ldots \ldots \ldots \ldots \ldots \ldots$ P6

Weaver I . . . . . . . . . . . . . . . . 23,23A

Weaver RR ................. P26

Webb J ..................... P66
Weinrib A ................21,21C

Weir BS . . . . . . . . . . . . . P77

Wideman TH $\ldots \ldots \ldots \ldots \ldots \ldots$. $18,18 \mathrm{~A}$

Wilson AC ................... P23

$\mathrm{Y}$
Yakobov E ................ P70

Yau B .................... P88

Yohannes L . . . . . . . . . . . . . . . . P65

Young A .................... P66

\section{Z}

Zamponi GW $\ldots \ldots \ldots \ldots \ldots \ldots .5 \mathrm{C}$

Zoheiry $\mathrm{N} \ldots \ldots \ldots \ldots \ldots \ldots . . . \ldots \ldots$

Zylka M .....................15 


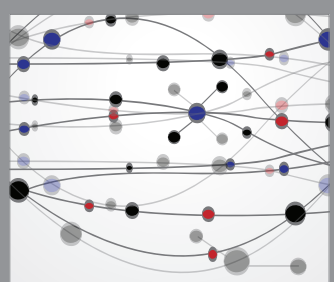

The Scientific World Journal
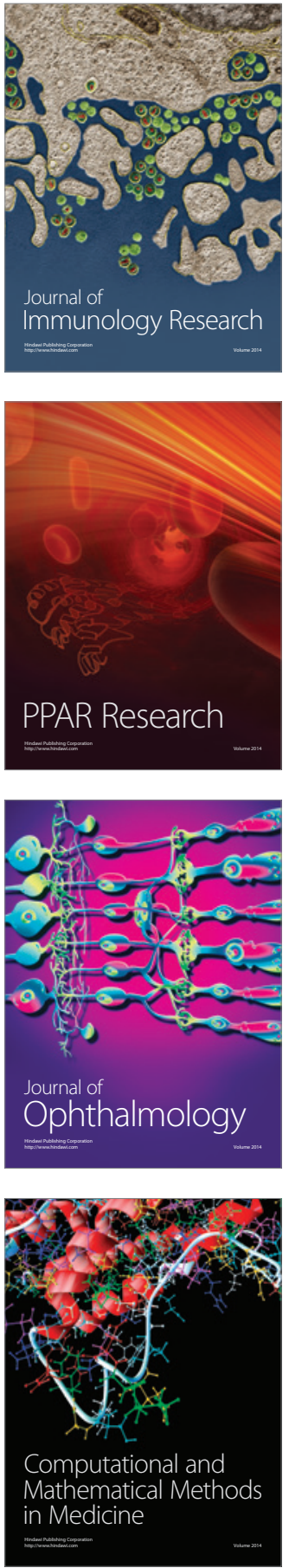

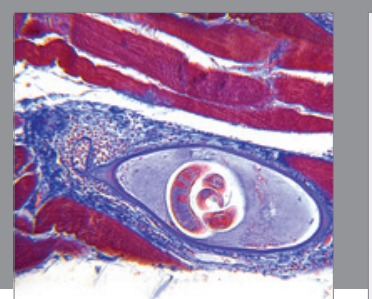

Gastroenterology Research and Practice

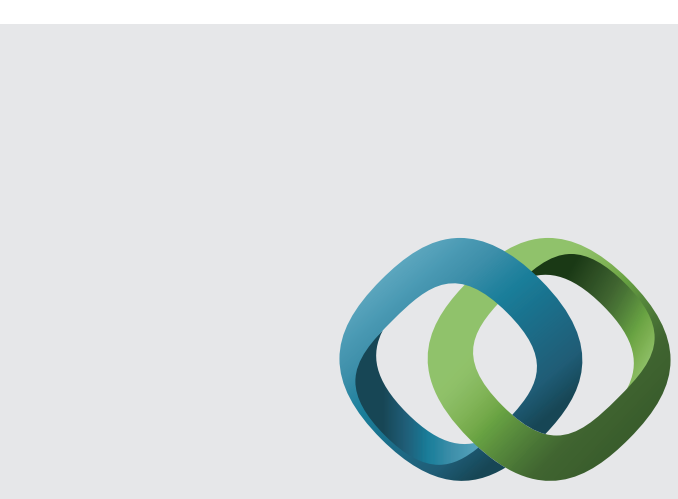

\section{Hindawi}

Submit your manuscripts at

http://www.hindawi.com
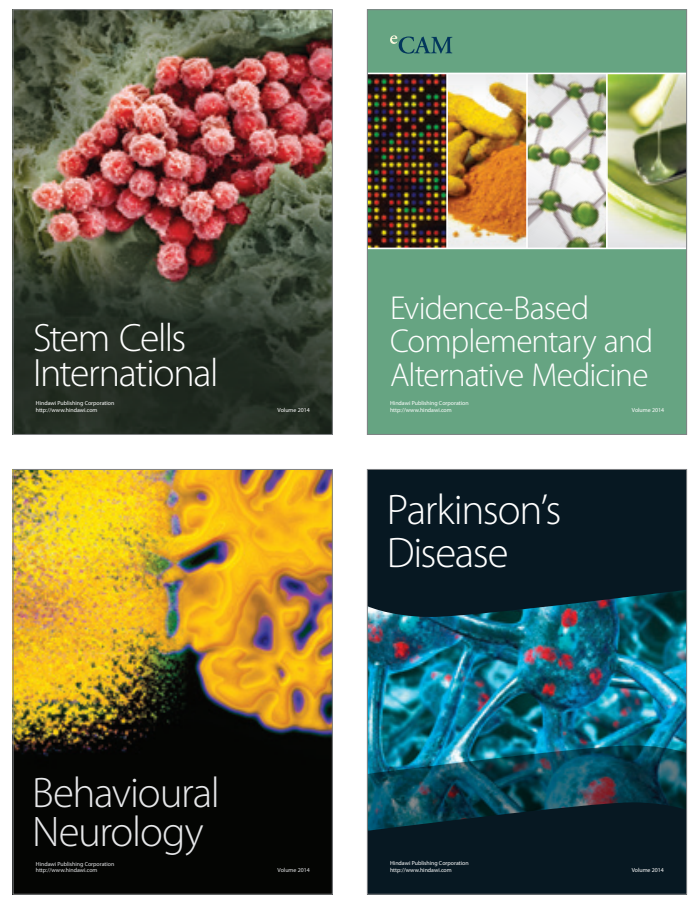
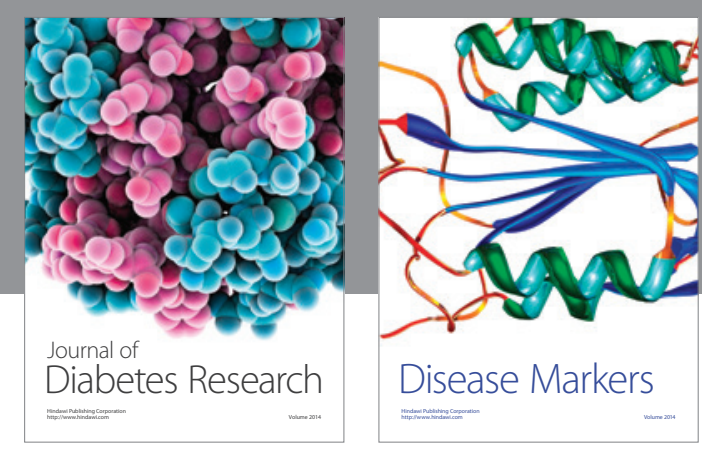

Disease Markers
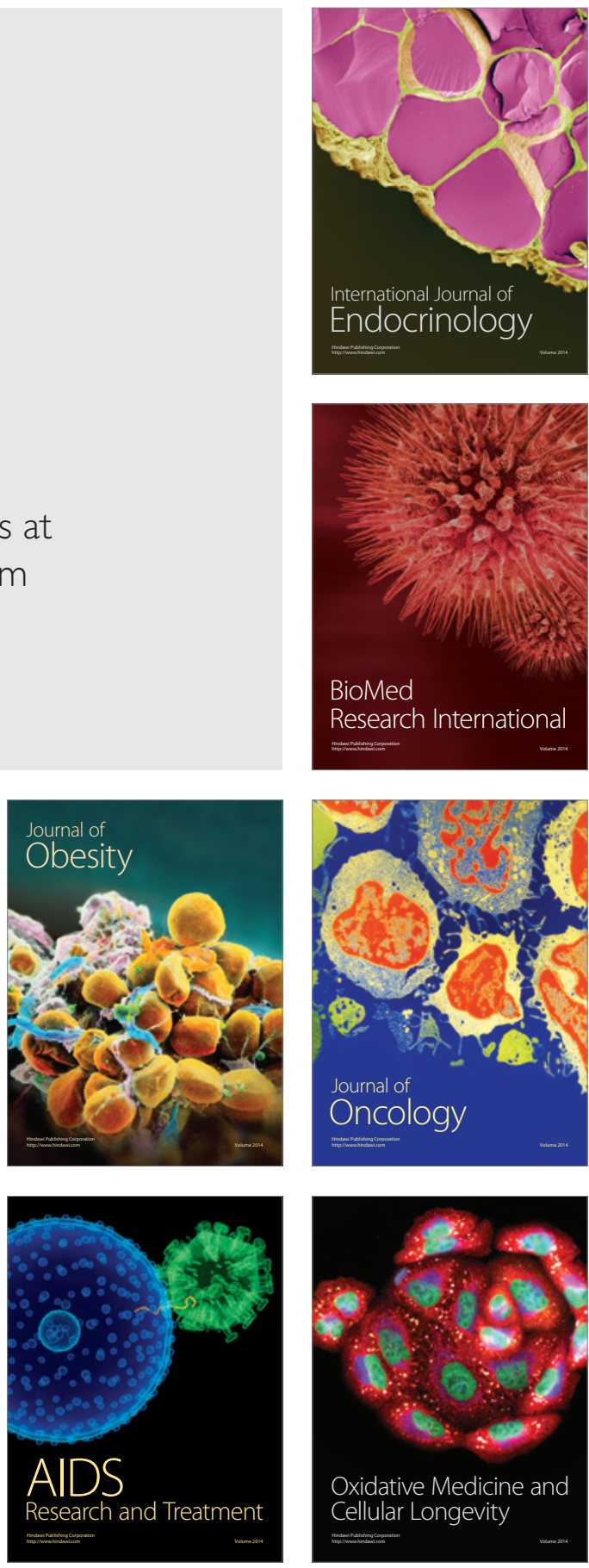